\title{
Accounting for Strong Ligand Sensitivity in Pd-Catalyzed $\alpha-$ Arylation of Enolates from Ketones, Esters, and Nitroalkanes
}

\author{
Sergei Tcyrulnikov, Marisa C. Kozlowski*
}

Table of Contents:

Computational details

S1

Coordinates and thermochemical data

S2

\section{Computational details}

Optimizations of intermediates and transition states were performed using Gaussian $09^{1}$ software with spin-restricted DFT using the B3LYP functional ${ }^{2}$ and a 6-31G(d) /LANL2DZ: Pd, Br) basis set in the gas phase. For all species, vibrational frequencies were also computed at the specified level of theory to obtain thermal Gibbs Free Energy corrections (at $298 \mathrm{~K}$ ) and to characterize the stationary points as transition states (one and only one imaginary frequency) or minima (zero imaginary frequencies). Single point energy calculations were performed on optimized gas phase geometries in solvent (toluene) using the SMD-solvation model ${ }^{3}$, M06 functional ${ }^{4}$ and 6-311+G(d,p)/ LANL2DZ:Pd, Br) split basis set. Singlepoint energies were converted to the enthalpies and Gibbs free energies using corrections from gas-phase

\footnotetext{
${ }^{1}$ Gaussian 09, Revision D.01, M. J. Frisch, G. W. Trucks, H. B. Schlegel, G. E. Scuseria, M. A. Robb, J. R. Cheeseman, G. Scalmani, V. Barone, B. Mennucci, G. A. Petersson, H. Nakatsuji, M. Caricato, X. Li, H. P. Hratchian, A. F. Izmaylov, J. Bloino, G. Zheng, J. L. Sonnenberg, M. Hada, M. Ehara, K. Toyota, R. Fukuda, J. Hasegawa, M. Ishida, T. Nakajima, Y. Honda, O. Kitao, H. Nakai, T. Vreven, J. A. Montgomery, Jr., J. E. Peralta, F. Ogliaro, M. Bearpark, J. J. Heyd, E. Brothers, K. N. Kudin, V. N. Staroverov, R. Kobayashi, J. Normand, K. Raghavachari, A. Rendell, J. C. Burant, S. S. Iyengar, J. Tomasi, M. Cossi, N. Rega, J. M. Millam, M. Klene, J. E. Knox, J. B. Cross, V. Bakken, C. Adamo, J. Jaramillo, R. Gomperts, R. E. Stratmann, O. Yazyev, A. J. Austin, R. Cammi, C. Pomelli, J. W. Ochterski, R. L. Martin, K. Morokuma, V. G. Zakrzewski, G. A. Voth, P. Salvador, J. J. Dannenberg, S. Dapprich, A. D. Daniels, Ö. Farkas, J. B. Foresman, J. V. Ortiz, J. Cioslowski, and D. J. Fox, Gaussian, Inc., Wallingford CT, 2009.

${ }^{2}$ A. D. Becke, 'A new mixing of Hartree-Fock and local density-functional theories', J. Chem. Phys., 1993, 98 (2), 1372-1377; C. Lee, W. Yang, and R. G. Parr, 'Development of the Colle-Salvetti correlation-energy formula into a functional of the electron density', Phys. Rev. B, 1988, 37 (2), 785-789.

${ }^{3}$ A. V Marenich, C. J. Cramer, and D. G. Truhlar, 'Universal Solvation Model Based on Solute Electron Density and on a Continuum Model of the Solvent Defined by the Bulk Dielectric Constant and Atomic Surface Tensions', J. Phys. Chem. B, 2009, 113 (18), 63786396.

${ }^{4}$ Y. Zhao and D. G. Truhlar, 'The M06 suite of density functionals for main group thermochemistry, thermochemical kinetics, noncovalent interactions, excited states, and transition elements: two new functionals and systematic testing of four M06-class functionals and 12 other function', Theor. Chem. Acc., 2008, 120 (1), 215-241.
} 
frequency analysis. Conformational analysis was performed manually. 3D structures were generated using CYLView program.

Coordinates and thermochemical data.

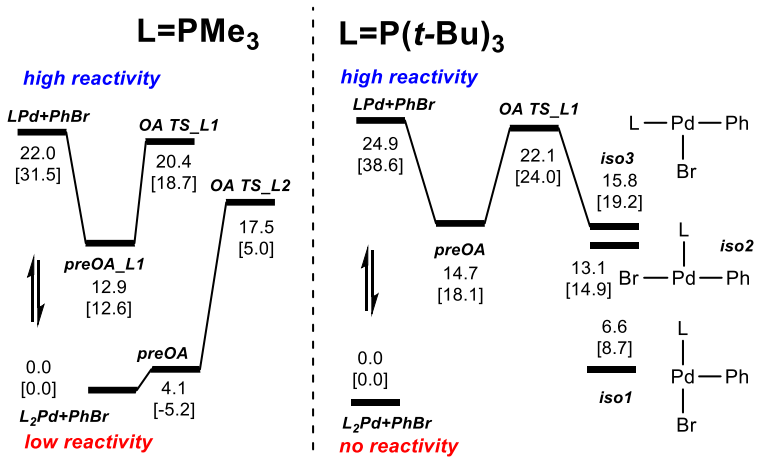

(PMe3)2Pd

Zero-point correction= (Hartree/Particle)

Thermal correction to Energy=

Thermal correction to Enthalpy=

0.229790

Thermal correction to Gibbs Free Energy $=\quad 0.182582 \mathrm{~s}$

Sum of electronic and zero-point Energies $=\quad-1048.779106$

Sum of electronic and thermal Energies $=\quad-1048.762143$

Sum of electronic and thermal Enthalpies $=\quad-1048.761199$

Sum of electronic and thermal Free Energies $=-1048.826313$

Electronic energy (M06): -1048.86749

$\begin{array}{lrrr}\mathrm{P} & 2.31666800 & 0.00036100 & 0.00005100 \\ \mathrm{C} & 3.17255000 & -1.31906000 & -0.98804000 \\ \mathrm{H} & 4.26515600 & -1.23802200 & -0.92594400 \\ \mathrm{H} & 2.86573700 & -2.30351100 & -0.62084200 \\ \mathrm{H} & 2.86706200 & -1.24317800 & -2.03634800 \\ \mathrm{C} & 3.16656800 & -0.19067200 & 1.64047600 \\ \mathrm{H} & 2.86137100 & -1.13655500 & 2.09896900 \\ \mathrm{H} & 4.25955100 & -0.17544100 & 1.54379500 \\ \mathrm{H} & 2.85508900 & 0.61974700 & 2.30700300 \\ \mathrm{C} & 3.15711100 & 1.52306800 & -0.65153000 \\ \mathrm{H} & 2.84291400 & 2.39064400 & -0.06274200 \\ \mathrm{H} & 4.25057400 & 1.44017100 & -0.61283000 \\ \mathrm{H} & 2.84777100 & 1.69248200 & -1.68772500 \\ \mathrm{Pd} & -0.00006100 & -0.00543100 & -0.00066900 \\ \mathrm{P} & -2.31664500 & 0.00018200 & 0.00041900 \\ \mathrm{C} & -3.17208700 & -1.59303600 & 0.42369900 \\ \mathrm{H} & -2.86415600 & -2.36882800 & -0.28441300 \\ \mathrm{H} & -2.86710000 & -1.91398000 & 1.42470300 \\ \mathrm{H} & -4.26475500 & -1.49546100 & 0.39578800 \\ \mathrm{C} & -3.15931400 & 1.16968900 & 1.17174700 \\ \mathrm{H} & -2.84746200 & 2.19462300 & 0.94730800 \\ \mathrm{H} & -4.25264400 & 1.10491400 & 1.10542500 \\ \mathrm{H} & -2.84915800 & 0.94314500 & 2.19670700 \\ \mathrm{C} & -3.16457700 & 0.43534000 & -1.59377600 \\ \mathrm{H} & -4.25767500 & 0.41208800 & -1.49987400 \\ \mathrm{H} & -2.85391700 & 1.43668300 & -1.90815500 \\ \mathrm{H} & -2.85738500 & -0.26982700 & -2.37254200\end{array}$

\section{(PMe3)Pd}

Zero-point correction= 0.115011 (Hartree/Particle) Thermal correction to Energy=

Thermal correction to Enthalpy=
0.124405
0.123461
Thermal correction to Gibbs Free Energy= Sum of electronic and zero-point Energies= Sum of electronic and thermal Energies= Sum of electronic and thermal Enthalpies= Sum of electronic and thermal Free Energie Electronic energy (M06): -587.7965517

$\begin{array}{lrrr}\mathrm{Pd} & -1.33177500 & 0.00004200 & 0.00006900 \\ \mathrm{P} & 0.88111900 & -0.00014600 & -0.00023500 \\ \mathrm{C} & 1.72599900 & -1.56027000 & -0.54635000 \\ \mathrm{H} & 1.41985700 & -2.38646500 & 0.10241100 \\ \mathrm{H} & 1.41774400 & -1.80328600 & -1.56766700 \\ \mathrm{H} & 2.81899700 & -1.46337600 & -0.51394500 \\ \mathrm{C} & 1.72616100 & 1.25343200 & -1.07760200 \\ \mathrm{H} & 1.41752100 & 2.25917900 & -0.77690900 \\ \mathrm{H} & 2.81917500 & 1.17721400 & -1.00923800 \\ \mathrm{H} & 1.42068100 & 1.10532600 & -2.11774800 \\ \mathrm{C} & 1.72652900 & 0.30682100 & 1.62388600 \\ \mathrm{H} & 2.81955800 & 0.28892400 & 1.52293100 \\ \mathrm{H} & 1.41875500 & 1.28056700 & 2.01667200 \\ \mathrm{H} & 1.42042100 & -0.45773500 & 2.34424800\end{array}$

PMe3

Zero-point correction $=\quad 0.113363($ Hartree/Particle $)$ Thermal correction to Energy= $\quad 0.120096$

Thermal correction to Enthalpy= $\quad 0.121041$

Thermal correction to Gibbs Free Energy $=\quad 0.083980$

Sum of electronic and zero-point Energies $=\quad-460.985036$

Sum of electronic and thermal Energies $=\quad-460.978303$

Sum of electronic and thermal Enthalpies $=\quad-460.977358$

Sum of electronic and thermal Free Energies $=-461.014419$

Electronic energy (M06): -461.0185549

$\begin{array}{lrrr}\mathrm{P} & -0.00013900 & -0.00024800 & -0.60576400 \\ \mathrm{C} & -1.54553000 & -0.55971600 & 0.28091000 \\ \mathrm{H} & -1.44463400 & -0.52213500 & 1.37294500 \\ \mathrm{H} & -2.38805200 & 0.07482800 & -0.01498900 \\ \mathrm{H} & -1.78626000 & -1.58702800 & -0.01356700 \\ \mathrm{C} & 1.25787500 & -1.05804300 & 0.28079000 \\ \mathrm{H} & 2.26756300 & -0.75375300 & -0.01580800 \\ \mathrm{H} & 1.17658800 & -0.98683900 & 1.37275700 \\ \mathrm{H} & 1.12879900 & -2.10556000 & -0.01258200 \\ \mathrm{C} & 0.28788200 & 1.61807600 & 0.28059600 \\ \mathrm{H} & 0.26911300 & 1.51163100 & 1.37258700 \\ \mathrm{H} & 1.25891100 & 2.03050000 & -0.01443100 \\ \mathrm{H} & -0.48130800 & 2.34017400 & -0.01423100\end{array}$

$\mathrm{PhBr}$

Zero-point correction $=\quad 0.090528($ Hartree/Particle $)$ Thermal correction to Energy $=\quad 0.096302$

Thermal correction to Enthalpy= $\quad 0.097247$

Thermal correction to Gibbs Free Energy $=\quad 0.059551$

Sum of electronic and zero-point Energies $=\quad-244.712128$

Sum of electronic and thermal Energies $=\quad-244.706354$

Sum of electronic and thermal Enthalpies $=\quad-244.705409$

Sum of electronic and thermal Free Energies $=-244.743105$ Electronic energy (M06): -244.6591789

C

$0.13400000 \quad-0.00000100 \quad 0.00041600$ 


$\begin{array}{lrrr}\mathrm{C} & 0.80775400 & 1.21798900 & 0.00039600 \\ \mathrm{C} & 0.80775700 & -1.21799100 & 0.00039600 \\ \mathrm{C} & 2.20519900 & 1.20819300 & -0.00001700 \\ \mathrm{H} & 0.25796800 & 2.15270400 & 0.00040200 \\ \mathrm{C} & 2.20520000 & -1.20819200 & -0.00001700 \\ \mathrm{H} & 0.25797100 & -2.15270600 & 0.00040000 \\ \mathrm{C} & 2.90482400 & 0.00000200 & -0.00033400 \\ \mathrm{H} & 2.74316800 & 2.15227600 & -0.00036400 \\ \mathrm{H} & 2.74317300 & -2.15227400 & -0.00036200 \\ \mathrm{H} & 3.99098400 & 0.00000200 & -0.00094300 \\ \mathrm{Br} & -1.83947600 & 0.00000000 & -0.00011900\end{array}$

\section{(PMe3)Pd-preOA}

Zero-point correction=

Thermal correction

Thermal correction to Enthalpy= $\quad 0.222786$

0.205427 (Hartree/Particle)

Thermal correction to Gibbs Free Energy= 0.157502

Sum of electronic and zero-point Energies $=\quad-832.486615$

$-832.470199$

Sum of electronic and thermal Enthalpies $=\quad-832.469255$

Sum of electronic and thermal Free Energies $=\quad-832.534540$

Electronic energy (M06): -832.4869786

$\begin{array}{lrrr}\mathrm{C} & 1.79566800 & -0.85544000 & -1.22916600 \\ \mathrm{C} & 1.89289300 & -2.25263300 & -1.21069800 \\ \mathrm{C} & 1.96617500 & -2.94711200 & -0.00054100 \\ \mathrm{C} & 1.89802600 & -2.25283800 & 1.21009100 \\ \mathrm{C} & 1.80064000 & -0.85576000 & 1.22918300 \\ \mathrm{C} & 1.76697000 & -0.15542900 & 0.00018100 \\ \mathrm{H} & 1.81931900 & -0.30722900 & -2.16418300 \\ \mathrm{H} & 1.92986100 & -2.79070100 & -2.15409200 \\ \mathrm{H} & 2.06561000 & -4.02868400 & -0.00088100 \\ \mathrm{H} & 1.93895800 & -2.79110100 & 2.15320900 \\ \mathrm{Pd} & -0.38215100 & -0.04705700 & 0.00093800 \\ \mathrm{Br} & 2.22996300 & 1.79996600 & -0.00066600 \\ \mathrm{H} & 1.82723600 & -0.30773200 & 2.16423100 \\ \mathrm{P} & -2.68089100 & 0.08529600 & 0.00077400 \\ \mathrm{C} & -3.59776800 & -0.94811100 & -1.23896100 \\ \mathrm{H} & -4.68332300 & -0.80444300 & -1.16981400 \\ \mathrm{H} & -3.26560200 & -0.68366200 & -2.24775200 \\ \mathrm{H} & -3.36443100 & -2.00480800 & -1.07548300 \\ \mathrm{C} & -3.56330400 & -0.37635300 & 1.56748300 \\ \mathrm{H} & -3.21119500 & 0.26223700 & 2.38370000 \\ \mathrm{H} & -4.65106400 & -0.26866800 & 1.47217300 \\ \mathrm{H} & -3.32700700 & -1.41311400 & 1.82654700 \\ \mathrm{C} & -3.40955500 & 1.76029400 & -0.33075600 \\ \mathrm{H} & -4.50618100 & 1.74053800 & -0.30695500 \\ \mathrm{H} & -3.04797200 & 2.47044000 & 0.41947500 \\ \mathrm{H} & -3.07909500 & 2.11361700 & -1.31252800\end{array}$

\section{(PMe3)Pd-OA-TS}

Zero-point correction= 0.205235 (Hartree/Particle) Thermal correction to Energy= $\quad 0.220954$ Thermal correction to Enthalpy= $\quad 0.221899$ Thermal correction to Gibbs Free Energy $=\quad 0.158821$ Sum of electronic and zero-point Energies $=\quad-832.481420$ Sum of electronic and thermal Energies $=\quad-832.465700$ Sum of electronic and thermal Enthalpies $=\quad-832.464756$ Sum of electronic and thermal Free Energies $=\quad-832.527833$ Electronic energy (M06): -832.4763275

$\begin{array}{lrrr}\text { C } & 2.17874600 & -0.60927500 & -1.22604700 \\ \text { C } & 3.06053100 & -1.69340700 & -1.21064100 \\ \text { C } & 3.50118200 & -2.23299000 & 0.00145400 \\ \text { C } & 3.05620700 & -1.69547300 & 1.21290500 \\ \text { C } & 2.17440200 & -0.61137400 & 1.22698700 \\ \text { C } & 1.71000400 & -0.10772900 & 0.00005300 \\ \text { H } & 1.86699400 & -0.15342600 & -2.15963300 \\ \text { H } & 3.41482400 & -2.10522600 & -2.15228700\end{array}$

$\begin{array}{llll}\mathrm{H} & 4.20138400 & -3.06358500 & 0.00199800 \\ \mathrm{H} & 3.40711200 & -2.10891500 & 2.15511300 \\ \mathrm{Pd} & -0.33009700 & -0.11114900 & -0.00334900 \\ \mathrm{Br} & 1.20893100 & 2.13670500 & 0.00121400 \\ \mathrm{H} & 1.85936700 & -0.15708300 & 2.16023700 \\ \mathrm{P} & -2.67340900 & -0.41484400 & -0.00081100 \\ \mathrm{C} & -3.39187700 & -1.43098900 & -1.37610300 \\ \mathrm{H} & -4.48241000 & -1.51396900 & -1.29553600 \\ \mathrm{H} & -3.13933200 & -0.97379400 & -2.33804400 \\ \mathrm{H} & -2.95480900 & -2.43430000 & -1.35575500 \\ \mathrm{C} & -3.40206700 & -1.24649900 & 1.48817300 \\ \mathrm{H} & -3.15204500 & -0.67154600 & 2.38534200 \\ \mathrm{H} & -4.49260500 & -1.33462500 & 1.41280400 \\ \mathrm{H} & -2.96935900 & -2.24596400 & 1.59719800 \\ \mathrm{C} & -3.70239800 & 1.12329900 & -0.10271100 \\ \mathrm{H} & -4.77611800 & 0.90039200 & -0.08904600 \\ \mathrm{H} & -3.45988900 & 1.77746700 & 0.74052900 \\ \mathrm{H} & -3.45847800 & 1.66203700 & -1.02360400\end{array}$

\section{(PMe3)2Pd-preOA}

Zero-point correction=

Thermal correction to Energy=

Thermal correction to Enthalpy=

0.321182 (Hartree/Particle)

Thermal correction to Gibbs Free Energy= $\quad 0.259040$

Sum of electronic and zero-point Energies $=\quad-1293.495275$

Sum of electronic and thermal Energies $=\quad-1293.470368$

Sum of electronic and thermal Enthalpies $=\quad-1293.469423$

Sum of electronic and thermal Free Energies $=-1293.557417$ Electronic energy (M06): -1293.536984

$\begin{array}{lrrr}\mathrm{C} & 1.83224100 & -0.13178700 & 0.64545100 \\ \mathrm{C} & 1.54922300 & -0.16327400 & 2.01484000 \\ \mathrm{C} & 2.58197700 & -0.22629100 & 2.95260900 \\ \mathrm{C} & 3.91011100 & -0.25836900 & 2.52192800 \\ \mathrm{C} & 4.21327400 & -0.22746900 & 1.15763400 \\ \mathrm{C} & 3.16387300 & -0.16439000 & 0.24505500 \\ \mathrm{H} & 1.01387500 & -0.08220600 & -0.06942700 \\ \mathrm{H} & 0.50750600 & -0.13672700 & 2.32554300 \\ \mathrm{H} & 2.35423300 & -0.25037000 & 4.01471100 \\ \mathrm{H} & 4.71984700 & -0.30741500 & 3.24512000 \\ \mathrm{Pd} & -1.85270000 & 0.09160400 & -0.02874900 \\ \mathrm{Br} & 3.58597300 & -0.11776000 & -1.68616400 \\ \mathrm{H} & 5.24219900 & -0.25172000 & 0.81589100 \\ \mathrm{P} & -2.09726700 & -2.21549000 & -0.10049200 \\ \mathrm{P} & -1.64208100 & 2.40470000 & -0.03737800 \\ \mathrm{C} & -0.37765800 & 3.11916800 & -1.19315600 \\ \mathrm{H} & -0.34896900 & 4.21482600 & -1.14391900 \\ \mathrm{H} & 0.61174400 & 2.72369500 & -0.94269500 \\ \mathrm{H} & -0.60957800 & 2.81349700 & -2.21820400 \\ \mathrm{C} & -3.14903100 & 3.37900100 & -0.51487000 \\ \mathrm{H} & -3.96424700 & 3.15177900 & 0.17922100 \\ \mathrm{H} & -2.95894200 & 4.45959400 & -0.50381700 \\ \mathrm{H} & -3.47043100 & 3.08244300 & -1.51814100 \\ \mathrm{C} & -1.17520300 & 3.23898800 & 1.55431600 \\ \mathrm{H} & -1.09944700 & 4.32747400 & 1.43996200 \\ \mathrm{H} & -1.92490300 & 3.01173400 & 2.31889800 \\ \mathrm{H} & -0.21256700 & 2.84840700 & 1.89866100 \\ \mathrm{C} & -2.02116600 & -3.14622400 & 1.50456400 \\ \mathrm{H} & -2.16135500 & -4.22454400 & 1.35901600 \\ \mathrm{H} & -1.04944600 & -2.97400600 & 1.97802900 \\ \mathrm{H} & -2.79654400 & -2.77275100 & 2.18080100 \\ \mathrm{C} & -0.86273200 & -3.16459700 & -1.11097100 \\ \mathrm{H} & 0.14448800 & -2.98096200 & -0.72423000 \\ \mathrm{H} & -1.06190700 & -4.24345300 & -1.09394900 \\ \mathrm{H} & -0.89233100 & -2.81178100 & -2.14660200 \\ \mathrm{C} & -3.69287900 & -2.85653600 & -0.80172500 \\ \mathrm{H} & -3.72710200 & -3.95326900 & -0.80883200 \\ \mathrm{H} & -4.53023800 & -2.47588600 & -0.20852500 \\ \mathrm{H} & -3.81271500 & -2.48804500 & -1.82530600 \\ & & & \\ & & & \end{array}$

(PMe3)2Pd-OA_TS 
Zero-point correction=

$\begin{array}{lc}\text { Thermal correction to Energy= } & 0.343797 \\ \text { Thermal correction to Enthalpy= } & 0.344742 \\ \text { Thermal correction to Gibbs Free Energy= } & 0.261854 \\ \text { Sum of electronic and zero-point Energies }= & -1293.471651 \\ \text { Sum of electronic and thermal Energies= } & -1293.447352 \\ \text { Sum of electronic and thermal Enthalpies }= & -1293.446407 \\ \text { Sum of electronic and thermal Free Energies }= & -1293.529295\end{array}$

Electronic energy (M06): -1293.518436

$\begin{array}{lrrr}\mathrm{C} & 1.40805700 & -1.57310200 & 1.30704400 \\ \mathrm{C} & 2.36988300 & -1.46016100 & 2.32279600 \\ \mathrm{C} & 3.54157300 & -0.73824500 & 2.11230400 \\ \mathrm{C} & 3.76434000 & -0.12493800 & 0.86850500 \\ \mathrm{C} & 2.81519900 & -0.20425900 & -0.14520000 \\ \mathrm{C} & 1.60063400 & -0.86886000 & 0.10614000 \\ \mathrm{H} & 0.54453300 & -2.21693600 & 1.43298800 \\ \mathrm{H} & 2.20896200 & -1.98072700 & 3.26387000 \\ \mathrm{H} & 4.29658600 & -0.67996800 & 2.89108000 \\ \mathrm{H} & 4.69013100 & 0.41664400 & 0.68771000 \\ \mathrm{Pd} & -0.33237000 & 0.08038200 & 0.01460600 \\ \mathrm{Br} & 0.71481900 & -1.90386200 & -1.67855000 \\ \mathrm{H} & 2.99394700 & 0.25052400 & -1.11350200 \\ \mathrm{P} & -2.64712400 & -0.48869200 & 0.53370100 \\ \mathrm{P} & 0.26277900 & 2.37901400 & -0.29953100 \\ \mathrm{C} & 1.15749300 & 2.86321000 & -1.85565900 \\ \mathrm{H} & 1.39027700 & 3.93511700 & -1.88139100 \\ \mathrm{H} & 2.09067900 & 2.29749000 & -1.93429100 \\ \mathrm{H} & 0.53947000 & 2.61018100 & -2.72305700 \\ \mathrm{C} & -1.12483900 & 3.61699100 & -0.32497800 \\ \mathrm{H} & -1.68203900 & 3.55730700 & 0.61571400 \\ \mathrm{H} & -0.76250200 & 4.64411800 & -0.45754100 \\ \mathrm{H} & -1.81323700 & 3.37635400 & -1.14185000 \\ \mathrm{C} & 1.36772900 & 3.13061000 & 0.98917300 \\ \mathrm{H} & 1.62274000 & 4.17270100 & 0.75994400 \\ \mathrm{H} & 0.86752500 & 3.09122600 & 1.96236500 \\ \mathrm{H} & 2.28625800 & 2.54023900 & 1.06222100 \\ \mathrm{C} & -3.13609900 & -2.25265700 & 0.22265900 \\ \mathrm{H} & -4.20524200 & -2.42434600 & 0.39805000 \\ \mathrm{H} & -2.89130500 & -2.51693800 & -0.81061700 \\ \mathrm{H} & -2.55698700 & -2.91118700 & 0.87809300 \\ \mathrm{C} & -3.96291600 & 0.40425500 & -0.42691000 \\ \mathrm{H} & -3.80075400 & 0.24088900 & -1.49726600 \\ \mathrm{H} & -4.97168000 & 0.06323800 & -0.16270800 \\ \mathrm{H} & -3.88859000 & 1.47960200 & -0.23571700 \\ \mathrm{C} & -3.26592200 & -0.25336400 & 2.27040800 \\ \mathrm{H} & -4.31361200 & -0.55948200 & 2.38144100 \\ \mathrm{H} & -2.64931100 & -0.84029200 & 2.95881800 \\ \mathrm{H} & -3.17109100 & 0.80015400 & 2.55278000\end{array}$

\section{(PtBu3)2Pd}

Zero-point correction=

$\quad 0.746409$ (Hartree/Particle)

Thermal correction to Energy $=\quad 0.785425$

Thermal correction to Enthalpy $=\quad 0.786370$

Thermal correction to Gibbs Free Energy $=0.682183$

Sum of electronic and zero-point Energies $=\quad-1755.806974$

Sum of electronic and thermal Energies $=\quad-1755.767958$

Sum of electronic and thermal Enthalpies $=\quad-1755.767014$

Sum of electronic and thermal Free Energies -1755.871200

Electronic energy (M06): -1756.094767

$\begin{array}{lccc}\mathrm{P} & 0.00000000 & 0.00000000 & 2.36516500 \\ \mathrm{C} & -1.80824200 & 0.22953400 & 3.06374500 \\ \mathrm{C} & 1.10290400 & 1.45121600 & 3.06374500 \\ \mathrm{C} & 0.70533800 & -1.68075100 & 3.06374500 \\ \mathrm{Pd} & 0.00000000 & 0.00000000 & 0.00000000 \\ \mathrm{P} & 0.00000000 & 0.00000000 & -2.36516500 \\ \mathrm{C} & -1.10290400 & 1.45121600 & -3.06374500 \\ \mathrm{C} & 1.80824200 & 0.22953400 & -3.06374500 \\ \mathrm{C} & -0.70533800 & -1.68075100 & -3.06374500 \\ \mathrm{C} & 2.75985700 & -0.62843700 & -2.19404900\end{array}$

$\begin{array}{lll}3.79817300 & -0.39483000 & -2.46806100\end{array}$

$2.62018600-0.40321400-1.13128500$

$\begin{array}{lll}2.62058500 & -1.70144500 & -2.33022800\end{array}$

$\begin{array}{lll}2.25355300 & 1.69150200 & -2.83864700\end{array}$

$\begin{array}{lll}2.09485200 & 2.00473800 & -1.80166300\end{array}$

$\begin{array}{lll}3.32913600 & 1.76600700 & -3.04830000\end{array}$

$\begin{array}{lll}1.74959500 & 2.39950000 & -3.50032900\end{array}$

$2.02599200-0.12282700-4.54878800$

$\begin{array}{lll}3.06549900 & 0.10500800 & -4.82389200\end{array}$

$\begin{array}{lll}1.86949700 & -1.18488500 & -4.75372700\end{array}$

$\begin{array}{lll}1.37939700 & 0.45041300 & -5.21812800\end{array}$

$\begin{array}{lll}-0.83568600 & 2.70432400 & -2.19404900\end{array}$

$\begin{array}{lll}0.16320200 & 3.12021600 & -2.33022800\end{array}$

$\begin{array}{llll}-1.55715400 & 3.48673000 & -2.46806100\end{array}$

$\begin{array}{lll}-0.96089900 & 2.47075400 & -1.13128500\end{array}$

$\begin{array}{lll}-2.59166000 & 1.10588300 & -2.83864700\end{array}$

$\begin{array}{lll}-2.78358000 & 0.81182600 & -1.80166300\end{array}$

$\begin{array}{lll}-3.19397500 & 2.00011300 & -3.04830000\end{array}$

$\begin{array}{lll}-2.95282600 & 0.31544400 & -3.50032900\end{array}$

$\begin{array}{lll}-0.90662400 & 1.81597400 & -4.54878800\end{array}$

$\begin{array}{llll}-1.62368900 & 2.60229600 & -4.82389200\end{array}$

$\begin{array}{lll}0.09139200 & 2.21147500 & -4.75372700\end{array}$

$\begin{array}{lll}-1.07976800 & 0.96938600 & -5.21812800\end{array}$

$\begin{array}{lll}-1.92417100 & -2.07588700 & -2.19404900\end{array}$

$\begin{array}{lll}-2.78378700 & -1.41877100 & -2.33022800\end{array}$

$\begin{array}{lll}-2.24101900 & -3.09190000 & -2.46806100\end{array}$

$\begin{array}{lll}-1.65928700 & -2.06754000 & -1.13128500\end{array}$

$\begin{array}{lll}-1.11936700 & -1.69314700 & -4.54878800\end{array}$

$\begin{array}{lll}-1.44181000 & -2.70730400 & -4.82389200\end{array}$

$-1.96088900-1.02659000-4.75372700$

$\begin{array}{lll}-0.29962900 & -1.41979900 & -5.21812800\end{array}$

$\begin{array}{llll}0.33810700 & -2.79738500 & -2.83864700\end{array}$

$\begin{array}{lll}-0.13516100 & -3.76612000 & -3.04830000\end{array}$

$\begin{array}{llll}1.20323000 & -2.71494400 & -3.50032900\end{array}$

$\begin{array}{lll}0.68872800 & -2.81656400 & -1.80166300\end{array}$

$\begin{array}{lll}0.83568600 & 2.70432400 & 2.19404900\end{array}$

$\begin{array}{lll}1.55715400 & 3.48673000 & 2.46806100\end{array}$

$\begin{array}{lll}0.96089900 & 2.47075400 & 1.13128500\end{array}$

$\begin{array}{lll}-0.16320200 & 3.12021600 & 2.33022800\end{array}$

$\begin{array}{lll}2.59166000 & 1.10588300 & 2.83864700\end{array}$

$\begin{array}{lll}2.78358000 & 0.81182600 & 1.80166300\end{array}$

$\begin{array}{lll}3.19397500 & 2.00011300 & 3.04830000\end{array}$

$\begin{array}{lll}2.95282600 & 0.31544400 & 3.50032900\end{array}$

$\begin{array}{llll}0.90662400 & 1.81597400 & 4.54878800\end{array}$

$\begin{array}{lll}1.07976800 & 0.96938600 & 5.21812800\end{array}$

$\begin{array}{lll}1.62368900 & 2.60229600 & 4.82389200\end{array}$

$\begin{array}{lll}-0.09139200 & 2.21147500 & 4.75372700\end{array}$

$\begin{array}{lll}-0.33810700 & -2.79738500 & 2.83864700\end{array}$

$\begin{array}{lll}-0.68872800 & -2.81656400 & 1.80166300\end{array}$

$\begin{array}{lll}0.13516100 & -3.76612000 & 3.04830000\end{array}$

$\begin{array}{lll}-1.20323000 & -2.71494400 & 3.50032900\end{array}$

$\begin{array}{lll}1.92417100 & -2.07588700 & 2.19404900\end{array}$

$\begin{array}{lll}2.78378700 & -1.41877100 & 2.33022800\end{array}$

$\begin{array}{lll}2.24101900 & -3.09190000 & 2.46806100\end{array}$

$\begin{array}{lll}1.65928700 & -2.06754000 & 1.13128500\end{array}$

$\begin{array}{lll}1.11936700 & -1.69314700 & 4.54878800\end{array}$

$\begin{array}{lll}1.44181000 & -2.70730400 & 4.82389200\end{array}$

$\begin{array}{lll}1.96088900 & -1.02659000 & 4.75372700\end{array}$

$\begin{array}{llll}0.29962900 & -1.41979900 & 5.21812800\end{array}$

$\begin{array}{lll}-2.25355300 & 1.69150200 & 2.83864700\end{array}$

$\begin{array}{lll}-3.32913600 & 1.76600700 & 3.04830000\end{array}$

$\begin{array}{lll}-1.74959500 & 2.39950000 & 3.50032900\end{array}$

$\begin{array}{lll}-2.09485200 & 2.00473800 & 1.80166300\end{array}$

$\begin{array}{lll}-2.75985700 & -0.62843700 & 2.19404900\end{array}$

$\begin{array}{lll}-2.62058500 & -1.70144500 & 2.33022800\end{array}$

$\begin{array}{lll}-3.79817300 & -0.39483000 & 2.46806100\end{array}$

$\begin{array}{lll}-2.62018600 & -0.40321400 & 1.13128500\end{array}$

$\begin{array}{lll}-2.02599200 & -0.12282700 & 4.54878800\end{array}$

$\begin{array}{lll}-3.06549900 & 0.10500800 & 4.82389200\end{array}$

$\begin{array}{lll}-1.86949700 & -1.18488500 & 4.75372700\end{array}$ 


\section{(PtBu3)Pd}

Zero-point correction $=$

Thermal correction to Energy=

Thermal correction to Enthalpy=

0.372414 (Hartree/Particle)

Thermal correction to Gibbs Free Energy=

Sum of electronic and zero-point Energies=

Sum of electronic and thermal Energies=

Sum of electronic and thermal Enthalpies=

Sum of electronic and thermal Free Energies $=-941.310112$

Electronic energy (M06): -941.4070967

$\begin{array}{llll}\mathrm{Pd} & -2.10961200 & 0.00016800 & 0.01152300\end{array}$

$\begin{array}{llll}P & 0.14796500 & 0.00020800 & -0.00057500\end{array}$

$\begin{array}{llll}\text { C } & 0.85133900 & 0.45543200 & 1.76403000\end{array}$

C $\quad 0.83540300-1.75993000-0.49412100$

$\begin{array}{llll}\text { C } & 0.82989900 & 1.30504600 & -1.28409300\end{array}$

C $\quad-0.03483600-2.30668400-1.65145300$

$\mathrm{H} \quad 0.26320300 \quad-3.34428900-1.85634000$

$\mathrm{H} \quad-1.09485100 \quad-2.29693300-1.37602700$

$\mathrm{H} \quad \begin{array}{llll}\mathrm{H} & 0.07538200 & -1.74589100 & -2.57994700\end{array}$

$\begin{array}{llll}\text { C } & 0.61783100 & -2.73657600 & 0.68253000\end{array}$

$\begin{array}{llll}\mathrm{H} & -0.41738300 & -2.70987800 & 1.03836400\end{array}$

$\begin{array}{llll}\mathrm{H} & 0.82386800 & -3.75661000 & 0.33215200\end{array}$

H $\quad \begin{array}{llll}H & 1.28487200 & -2.54722900 & 1.52662600\end{array}$

C $\quad 2.31960900-1.81815700 \quad-0.91118900$

$\mathrm{H} \quad 2.59252700-2.86339000 \quad-1.11271900$

$\mathrm{H} \quad 2.52037600-1.25570200-1.82674000$

$\mathrm{H} \quad 2.99372500-1.45209500 \quad-0.13255300$

$\begin{array}{llll}\text { C } & -0.00936100 & -0.27447000 & 2.82327800\end{array}$

$\begin{array}{llll}\mathrm{H} & 0.09787600 & -1.35912900 & 2.79707900\end{array}$

$\begin{array}{llll}\mathrm{H} & 0.29911000 & 0.06353500 & 3.82227200\end{array}$

$\mathrm{H} \quad-1.07015600 \quad-0.03821200 \quad 2.68773900$

$\begin{array}{llll}\text { C } & 0.63597000 & 1.96263000 & 2.02462700\end{array}$

$\begin{array}{llll}\mathrm{H} & -0.40080500 & 2.25890400 & 1.83465900\end{array}$

$\mathrm{H} \quad 0.85293100 \quad 2.16844300 \quad 3.08125300$

$\mathrm{H} \quad \begin{array}{llll}\mathrm{H} & 1.29787000 & 2.59895200 & 1.43260700\end{array}$

$\begin{array}{llll}\text { C } & 2.33841400 & 0.12550500 & 2.00899800\end{array}$

$\begin{array}{llll}\mathrm{H} & 2.62068400 & 0.47537600 & 3.01170500\end{array}$

H $\quad 2.54073100-0.94821700 \quad 1.97914700$

H $\quad 3.00443300 \quad 0.61665300 \quad 1.29502700$

C $\quad-0.03378100 \quad 2.58393000 \quad-1.16874700$

H $\quad 0.09675600 \quad 3.11097600 \quad-0.22348100$

H $\quad 0.25073100 \quad 3.27558400 \quad-1.97369100$

$\mathrm{H} \quad-1.09668400 \quad 2.34258800 \quad-1.27590500$

C $\quad 2.31732200 \quad 1.68772900-1.14059800$

$\begin{array}{llll}\mathrm{H} & 2.58675900 & 2.38162700 & -1.94892700\end{array}$

$\mathrm{H} \quad 2.52864100 \quad 2.20031200 \quad-0.19864000$

$\mathrm{H} \quad 2.98663600 \quad 0.82688400 \quad-1.21644700$

C $\quad 0.59487600 \quad 0.77493500 \quad-2.71539800$

$\begin{array}{llll}\mathrm{H} & 0.79781900 & 1.58652100 & -3.42668200\end{array}$

H $\quad 1.25495200-0.05439800 \quad-2.97953800$

\section{(PtBu3)Pd-preOA}

Zero-point correction=

Thermal correction to Energy $\quad 0.463452$ (Hartree/Particle)

0.491113

Thermal correction to Enthalpy= $\quad 0.492057$

Thermal correction to Gibbs Free Energy $=\quad 0.404953$

Sum of electronic and zero-point Energies $=\quad-1186.001342$

Sum of electronic and thermal Energies $=\quad-1185.973681$

Sum of electronic and thermal Enthalpies $=\quad-1185.972737$

Sum of electronic and thermal Free Energies $=-1186.059841$

Electronic energy (M06): -1186.100584

$\begin{array}{llll}\text { C } & -2.87532000 & 0.86117300 & -1.22837100\end{array}$

C $\quad-3.01322100 \quad 2.25537600 \quad-1.20933500$

C $\quad-3.11001700 \quad 2.94683300 \quad 0.00095500$

$\begin{array}{llll}\text { C } & -3.02615500 & 2.25375200 & 1.21168200\end{array}$

C $\quad-2.88805400 \quad 0.86035100 \quad 1.23032200$

$\begin{array}{lrrr}\mathrm{C} & -2.82877800 & 0.15977100 & 0.00100300 \\ \mathrm{H} & -2.88676100 & 0.31281400 & -2.16362600 \\ \mathrm{H} & -3.06497100 & 2.79269900 & -2.15258500 \\ \mathrm{H} & -3.24266500 & 4.02487500 & 0.00093400 \\ \mathrm{H} & -3.08713900 & 2.79004100 & 2.15497300 \\ \mathrm{Pd} & -0.69408500 & 0.08940900 & -0.00405600 \\ \mathrm{Br} & -3.28594100 & -1.80367600 & -0.00158000 \\ \mathrm{H} & -2.90547700 & 0.31118600 & 2.16510300 \\ \mathrm{P} & 1.65948100 & -0.02091400 & 0.00021200 \\ \mathrm{C} & 2.42891900 & 1.23313500 & -1.28088300 \\ \mathrm{C} & 2.37068400 & 0.39887700 & 1.76710300 \\ \mathrm{C} & 2.23613700 & -1.81977300 & -0.47969600 \\ \mathrm{C} & 2.23719600 & 1.91654700 & 2.02300200 \\ \mathrm{H} & 1.22176100 & 2.27132400 & 1.81857900 \\ \mathrm{H} & 2.44999000 & 2.11149800 & 3.08253200 \\ \mathrm{H} & 2.94261800 & 2.51388100 & 1.44064600 \\ \mathrm{C} & 1.45704800 & -0.27875800 & 2.81718300 \\ \mathrm{H} & 1.77110900 & 0.04376800 & 3.81936400 \\ \mathrm{H} & 0.41236400 & 0.01689300 & 2.67281900 \\ \mathrm{H} & 1.50379900 & -1.36768700 & 2.79562100 \\ \mathrm{C} & 3.83311800 & -0.01461300 & 2.02881100 \\ \mathrm{H} & 4.12800400 & 0.32512400 & 3.03137900 \\ \mathrm{H} & 3.97255900 & -1.09856700 & 2.00700500 \\ \mathrm{H} & 4.53198300 & 0.43078400 & 1.31616000 \\ \mathrm{C} & 3.71282900 & -1.97357300 & -0.89393400 \\ \mathrm{H} & 4.40495900 & -1.63659400 & -0.11831200 \\ \mathrm{H} & 3.92347400 & -3.03525800 & -1.08281600 \\ \mathrm{H} & 3.94701400 & -1.43404200 & -1.81491800 \\ \mathrm{C} & 1.33028700 & -2.31144800 & -1.63566800 \\ \mathrm{H} & 1.57076000 & -3.36200000 & -1.84945100 \\ \mathrm{H} & 0.27267200 & -2.25294900 & -1.35648500 \\ \mathrm{H} & 1.46485000 & -1.75135100 & -2.56124800 \\ \mathrm{C} & 1.95510700 & -2.77970100 & 0.69765500 \\ \mathrm{H} & 2.09623100 & -3.81043900 & 0.34646800 \\ \mathrm{H} & 2.63263800 & -2.63422800 & 1.54216800 \\ \mathrm{H} & 0.92329100 & -2.69174000 & 1.05297000 \\ \mathrm{H} & 2.16947400 & 0.71722300 & -2.71363200 \\ \mathrm{C} & 2.42809400 & 1.51423400 & -3.42326700 \\ \mathrm{H} & 2.77687800 & -0.15230900 & -2.97425800 \\ \mathrm{H} & 1.11417400 & 0.46827000 & -2.86663700 \\ \mathrm{H} & 1.64408100 & 2.56347600 & -1.17488800 \\ \mathrm{H} & 1.79756700 & 3.08334600 & -0.22913500 \\ \mathrm{C} & 0.568946000 & 3.23523400 & -1.97651900 \\ \mathrm{H} & 2.39490300 & -1.29460200 \\ \mathrm{H} & 4.55037200 & 1.52350600 & -1.13071700 \\ \mathrm{H} & 0.62225000 & -1.20509000 \\ \mathrm{H} & 2.20117100 & -1.93609500 \\ \mathrm{H} & 2.01951700 & -0.18649400\end{array}$

(PtBu3)Pd-OA_TS first order transition state (singlenegative frequency)was located with geometrical constraints.

Zero-point correction=

$\begin{array}{ll}\text { Thermal correction to Energy= } & 0.490394 \\ \text { Thermal correction to Enthalpy= } & 0.491338\end{array}$

Thermal correction to Gibbs Free Energy $=\quad 0.406588$

Sum of electronic and zero-point Energies $=\quad-1185.996963$

Sum of electronic and thermal Energies $=\quad-1185.970106$

Sum of electronic and thermal Enthalpies $=\quad-1185.969162$

Sum of electronic and thermal Free Energies $=-1186.053912$

Electronic energy (M06): -1186.090532

$\begin{array}{llll}\text { C } & 3.20840000 & -0.68537200 & -1.23203500 \\ \text { C } & 4.02153700 & -1.82194700 & -1.21536300 \\ \text { C } & 4.42334000 & -2.38958300 & -0.00262400 \\ \text { C } & 4.00887300 & -1.82467400 & 1.20751600 \\ \text { C } & 3.19532200 & -0.68882000 & 1.21871200 \\ \text { C } & 2.76415800 & -0.15546500 & -0.00831200 \\ \text { H } & 2.93098600 & -0.20952900 & -2.16672400 \\ \text { H } & 4.35395600 & -2.25320100 & -2.15640800\end{array}$




$\begin{array}{lrrr}\mathrm{H} & 5.07163400 & -3.26132600 & -0.00022100 \\ \mathrm{H} & 4.33159800 & -2.25828700 & 2.15084100 \\ \mathrm{Pd} & 0.73086500 & 0.00982900 & -0.02916000 \\ \mathrm{Br} & 2.45033200 & 2.12270300 & -0.00325000 \\ \mathrm{H} & 2.90706400 & -0.21630400 & 2.15176100 \\ \mathrm{P} & -1.67623100 & -0.13818000 & -0.00519900 \\ \mathrm{C} & -2.27696500 & -1.66634400 & -1.05223800 \\ \mathrm{C} & -2.28235000 & -0.36327700 & 1.83088300 \\ \mathrm{C} & -2.48142100 & 1.47552900 & -0.73629600 \\ \mathrm{C} & -1.95124800 & -1.79471500 & 2.30902200 \\ \mathrm{H} & -0.90230000 & -2.05204500 & 2.12816300 \\ \mathrm{H} & -2.12039200 & -1.84745200 & 3.39257000 \\ \mathrm{H} & -2.58390700 & -2.55748700 & 1.84971400 \\ \mathrm{C} & -1.43689300 & 0.57768100 & 2.72365800 \\ \mathrm{H} & -1.68596300 & 0.38396900 & 3.77576800 \\ \mathrm{H} & -0.36631700 & 0.39090600 & 2.58612200 \\ \mathrm{H} & -1.61714300 & 1.63560200 & 2.53404200 \\ \mathrm{C} & -3.77919800 & -0.09807000 & 2.08890800 \\ \mathrm{H} & -4.00294100 & -0.30814300 & 3.14387400 \\ \mathrm{H} & -4.05873600 & 0.94254800 & 1.90637200 \\ \mathrm{H} & -4.43127700 & -0.73484200 & 1.48546700 \\ \mathrm{C} & -3.97234000 & 1.37187400 & -1.11477200 \\ \mathrm{H} & -4.60291100 & 1.08442400 & -0.26941000 \\ \mathrm{H} & -4.32139200 & 2.35294800 & -1.46501900 \\ \mathrm{H} & -4.14981000 & 0.66233100 & -1.92718300 \\ \mathrm{C} & -1.66459800 & 1.88913200 & -1.98496900 \\ \mathrm{H} & -2.03841000 & 2.85810000 & -2.34257200 \\ \mathrm{H} & -0.60349300 & 2.00583800 & -1.74146100 \\ \mathrm{H} & -1.74843700 & 1.18433800 & -2.81254000 \\ \mathrm{C} & -2.30743700 & 2.63384600 & 0.27143200 \\ \mathrm{H} & -2.59934100 & 3.56928000 & -0.22342400 \\ \mathrm{H} & -2.93898900 & 2.52990000 & 1.15651400 \\ \mathrm{H} & -1.26533800 & 2.74268900 & 0.58861500 \\ \mathrm{C} & -2.11569500 & -1.35022300 & -2.55587300 \\ \mathrm{H} & -2.27560600 & -2.27547000 & -3.12482900 \\ \mathrm{H} & -2.84100400 & -0.62005300 & -2.92106100 \\ \mathrm{H} & -1.10793000 & -0.99111700 & -2.78947300 \\ \mathrm{C} & -1.30852500 & -2.84102900 & -0.77095300 \\ \mathrm{H} & -1.37033200 & -3.22176400 & 0.24867100 \\ \mathrm{H} & -1.55473500 & -3.67158600 & -1.44635700 \\ \mathrm{H} & -0.26957000 & -2.54813600 & -0.95974200 \\ \mathrm{C} & -3.72556300 & -2.13203600 & -0.80315800 \\ \mathrm{H} & -4.46033200 & -1.34655600 & -0.99862000 \\ \mathrm{H} & -3.95409100 & -2.96838800 & -1.47801100 \\ \mathrm{H} & -3.87889100 & -2.49427400 & 0.21643200\end{array}$

Iso1

Zero-point correction= $\begin{array}{lc}\text { Thermal correction to Energy= } & 0.492080 \\ \text { Thermal correction to Enthalpy= } & 0.493024 \\ \text { Thermal correction to Gibbs Free Energy= } & 0.407842 \\ \text { Sum of electronic and zero-point Energies }= & -1186.024297 \\ \text { Sum of electronic and thermal Energies }= & -1185.996803\end{array}$ Sum of electronic and thermal Enthalpies $=\quad-1185.995859$ Sum of electronic and thermal Free Energies $=-1186.081040$ Electronic energy (M06): -1186.116455

$\begin{array}{llrr}\mathrm{C} & 2.05649500 & 1.67058700 & 1.19462100 \\ \mathrm{C} & 2.85102600 & 2.82446400 & 1.16369100 \\ \mathrm{C} & 3.42042500 & 3.26202500 & -0.03212700 \\ \mathrm{C} & 3.20584300 & 2.53210800 & -1.20243200 \\ \mathrm{C} & 2.41655400 & 1.37635800 & -1.18469100 \\ \mathrm{C} & 1.82674500 & 0.96338200 & 0.01372700 \\ \mathrm{H} & 1.64269900 & 1.33508800 & 2.13829200 \\ \mathrm{H} & 3.02690000 & 3.37205400 & 2.08660200 \\ \mathrm{H} & 4.03836200 & 4.15563700 & -0.04970200 \\ \mathrm{H} & 3.66247300 & 2.84864800 & -2.13707800 \\ \mathrm{Pd} & 0.77342200 & -0.72696500 & -0.00722400 \\ \mathrm{Br} & 2.83386000 & -2.17501800 & 0.05704700 \\ \mathrm{H} & 2.28363700 & 0.80137900 & -2.09522900\end{array}$

$\begin{array}{rrr}-1.47170800 & 0.09827700 & -0.01780700 \\ -2.14207500 & 0.01755900 & 1.80858800 \\ -1.88726600 & 1.83456200 & -0.77798600 \\ -2.34170300 & -1.29267700 & -1.06728500 \\ -3.37380900 & 2.02467700 & -1.14682300 \\ -3.51423200 & 3.05425600 & -1.50163700 \\ -3.69037600 & 1.36284400 & -1.95659800 \\ -4.04857300 & 1.87736300 & -0.30074800 \\ -1.47468200 & 2.95011400 & 0.20951800 \\ -1.57867900 & 3.91340200 & -0.30606700 \\ -2.10936100 & 2.99405000 & 1.09747300 \\ -0.43043400 & 2.85789100 & 0.52088200 \\ -1.02957100 & 2.05060700 & -2.04702900 \\ -1.24496800 & 1.34054300 & -2.84484500 \\ -1.24758500 & 3.05367100 & -2.43694000 \\ 0.03743100 & 2.00644300 & -1.82301000 \\ -3.85893900 & -1.44707800 & -0.83738300 \\ -4.41379200 & -0.53151000 & -1.05708900 \\ -4.23824400 & -2.22844800 & -1.50956600 \\ -4.10260300 & -1.75699700 & 0.18146500 \\ -1.63794600 & -2.63586000 & -0.74453100 \\ -2.07205300 & -3.42040800 & -1.37841700 \\ -0.56514800 & -2.60467300 & -0.98466800 \\ -1.74847900 & -2.95622600 & 0.29037200 \\ -2.09786300 & -1.04611500 & -2.57265200 \\ -2.43159300 & -1.93460200 & -3.12372800 \\ -2.66144900 & -0.19608000 & -2.96305800 \\ -1.03643200 & -0.90086600 & -2.79706800 \\ -3.54929900 & 0.61383500 & 2.02047300 \\ -4.31078800 & 0.14212300 & 1.39404600 \\ -3.84316000 & 0.45378100 & 3.06635600 \\ -3.57948900 & 1.69159500 & 1.84283200 \\ -1.13427900 & 0.75183600 & 2.72296800 \\ -0.99403500 & 1.80292800 & 2.47091000 \\ -1.50314400 & 0.70493700 & 3.75597700 \\ -0.15939600 & 0.25765800 & 2.69300400 \\ -2.14961800 & -1.44629200 & 2.30404000 \\ -2.36117000 & -1.43978400 & 3.38083900 \\ -2.91810500 & -2.06166400 & 1.83155500 \\ -1.17530200 & -1.92773400 & 2.16945600\end{array}$

Iso2 - METASTABLE

Zero-point correction=

Thermal correction to Energy=

0.464928 (Hartree/Particle)

Thermal correction to Enthalpy= $\quad 0.493267$

Thermal correction to Gibbs Free Energy $=\quad 0.408615$

Sum of electronic and zero-point Energies $=\quad-1186.015879$

Sum of electronic and thermal Energies $=\quad-1185.988484$

Sum of electronic and thermal Enthalpies $=\quad-1185.987539$

Sum of electronic and thermal Free Energies $=-1186.072192$ Electronic energy (M06): -1186.106913

$\begin{array}{lrrr}\mathrm{C} & -2.76025800 & -0.89408700 & 1.15931300 \\ \mathrm{C} & -4.05649200 & -1.42242600 & 1.19268600 \\ \mathrm{C} & -4.77482200 & -1.60836100 & 0.01038100 \\ \mathrm{C} & -4.19390900 & -1.26808300 & -1.21322900 \\ \mathrm{C} & -2.89833700 & -0.74113500 & -1.25954400 \\ \mathrm{C} & -2.18838400 & -0.53430300 & -0.06780400 \\ \mathrm{H} & -2.21657400 & -0.74875300 & 2.08855900 \\ \mathrm{H} & -4.50164500 & -1.68443300 & 2.14953600 \\ \mathrm{H} & -5.78131700 & -2.01644400 & 0.04168300 \\ \mathrm{H} & -4.74604200 & -1.41027000 & -2.13912400 \\ \mathrm{Pd} & -0.73147900 & 0.80728100 & -0.09879500 \\ \mathrm{Br} & -0.44082200 & 3.37312000 & 0.00573700 \\ \mathrm{H} & -2.45627400 & -0.48808000 & -2.21935800 \\ \mathrm{P} & 1.08715900 & -0.63595700 & -0.01522700 \\ \mathrm{C} & 1.87330200 & -0.22321300 & 1.72695800 \\ \mathrm{C} & 0.84599200 & -2.57188400 & -0.16090000 \\ \mathrm{C} & 2.30175100 & -0.05623300 & -1.42600200 \\ \mathrm{C} & 2.16818500 & -3.34851000 & -0.36865400\end{array}$




$\begin{array}{lrrr}\mathrm{H} & 1.94037100 & -4.42113400 & -0.31754100 \\ \mathrm{H} & 2.60994900 & -3.16583600 & -1.35135700 \\ \mathrm{H} & 2.92128400 & -3.14235100 & 0.39181000 \\ \mathrm{C} & 0.15719200 & -3.11558500 & 1.11039000 \\ \mathrm{H} & -0.07356000 & -4.17489900 & 0.94075100 \\ \mathrm{H} & 0.79165300 & -3.06462800 & 1.99787900 \\ \mathrm{H} & -0.78610300 & -2.60692900 & 1.31691900 \\ \mathrm{C} & -0.09285400 & -2.91527700 & -1.34040000 \\ \mathrm{H} & 0.28718000 & -2.60109600 & -2.31036000 \\ \mathrm{H} & -0.20129100 & -4.00724700 & -1.37373100 \\ \mathrm{H} & -1.08585400 & -2.48604300 & -1.20337000 \\ \mathrm{C} & 3.77922500 & -0.42529700 & -1.16476800 \\ \mathrm{H} & 3.94538500 & -1.49370500 & -1.01461000 \\ \mathrm{H} & 4.36333300 & -0.12537200 & -2.04433300 \\ \mathrm{H} & 4.19393900 & 0.11215000 & -0.30880700 \\ \mathrm{C} & 2.20893000 & 1.47932200 & -1.60204800 \\ \mathrm{H} & 2.91252100 & 1.76590700 & -2.39449100 \\ \mathrm{H} & 1.20983000 & 1.79916400 & -1.90494600 \\ \mathrm{H} & 2.46735500 & 2.04516100 & -0.70803600 \\ \mathrm{C} & 1.86849800 & -0.66734500 & -2.77807900 \\ \mathrm{H} & 2.43625700 & -0.16943800 & -3.57368700 \\ \mathrm{H} & 2.07871800 & -1.73552200 & -2.85771100 \\ \mathrm{H} & 0.80661500 & -0.49637000 & -2.98226600 \\ \mathrm{C} & 3.00023700 & -1.18208300 & 2.16880600 \\ \mathrm{H} & 3.82830900 & -1.22026900 & 1.45670900 \\ \mathrm{H} & 3.40679500 & -0.81246900 & 3.11914400 \\ \mathrm{H} & 2.64969400 & -2.20067000 & 2.34970000 \\ \mathrm{C} & 0.74575000 & -0.24214500 & 2.78384000 \\ \mathrm{H} & 0.25252800 & -1.21064100 & 2.88155800 \\ \mathrm{H} & 1.18380500 & 0.00602200 & 3.75916100 \\ \mathrm{H} & -0.01335200 & 0.51304000 & 2.55671500 \\ \mathrm{C} & 2.44328800 & 1.21324700 & 1.72294400 \\ \mathrm{H} & 2.72605300 & 1.46105600 & 2.75409800 \\ \mathrm{H} & 3.34489600 & 1.30921800 & 1.11372500 \\ \mathrm{H} & 1.71119500 & 1.95650300 & 1.39460400\end{array}$

\section{Iso3}

Zero-point correction= Thermal correction to Energy $=\quad 0.491309$ Thermal correction to Enthalpy= $\quad 0.492253$ Thermal correction to Gibbs Free Energy $=\quad 0.405002$ Sum of electronic and zero-point Energies $=\quad-1186.010677$ Sum of electronic and thermal Energies $=\quad-1185.982837$ Sum of electronic and thermal Enthalpies $=\quad-1185.981892$ Sum of electronic and thermal Free Energies $=-1186.069144$ Electronic energy (M06): -1186.098997

$\begin{array}{llrr}\mathrm{C} & -3.45370700 & -0.59803800 & 1.21099000 \\ \mathrm{C} & -4.71490300 & -1.20426900 & 1.21457500 \\ \mathrm{C} & -5.34807900 & -1.51692700 & 0.00908800 \\ \mathrm{C} & -4.72616200 & -1.21913900 & -1.20599000 \\ \mathrm{C} & -3.46491900 & -0.61294800 & -1.22198000 \\ \mathrm{C} & -2.81678800 & -0.35465000 & -0.01004500 \\ \mathrm{H} & -2.97796300 & -0.32286400 & 2.14860300 \\ \mathrm{H} & -5.20719200 & -1.41492900 & 2.16104500 \\ \mathrm{H} & -6.33461400 & -1.97293500 & 0.01653300 \\ \mathrm{H} & -5.22737100 & -1.44159900 & -2.14504600 \\ \mathrm{Pd} & -0.86141900 & -0.05054000 & -0.01815900 \\ \mathrm{Br} & -1.47619400 & 2.40236800 & -0.01051600 \\ \mathrm{H} & -2.99786000 & -0.35010600 & -2.16750700 \\ \mathrm{P} & 1.64513700 & -0.24080300 & -0.00255800 \\ \mathrm{C} & 2.29156800 & -0.15388800 & 1.82474400 \\ \mathrm{C} & 1.95661600 & -2.02745500 & -0.70762600 \\ \mathrm{C} & 2.59866100 & 1.05186000 & -1.08831000 \\ \mathrm{C} & 3.32276500 & -2.66179800 & -0.38182900 \\ \mathrm{H} & 3.46878000 & -2.81895600 & 0.68979400 \\ \mathrm{H} & 3.38684100 & -3.64732000 & -0.86309000 \\ \mathrm{H} & 4.15841900 & -2.06243900 & -0.75305300 \\ \mathrm{C} & 1.77435800 & -2.01811800 & -2.24174200 \\ \mathrm{H} & 1.77183900 & -3.05657300 & -2.59783700 \\ \mathrm{H} & 0.82088500 & -1.56785100 & -2.53737600\end{array}$

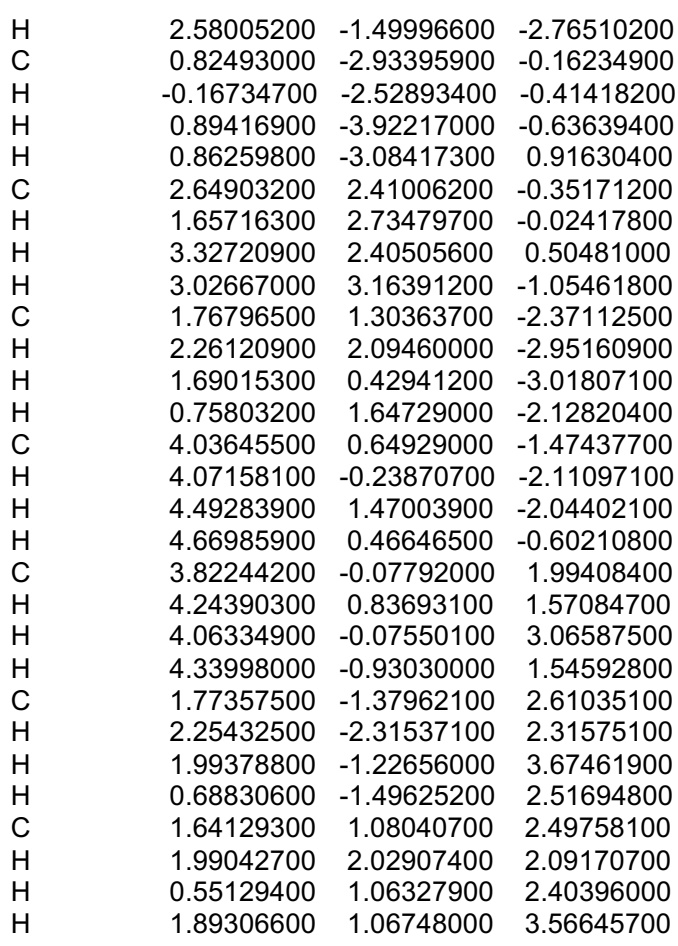

\section{TRANSMETALLATIONS}

\section{Reactivity of ketone enolate}

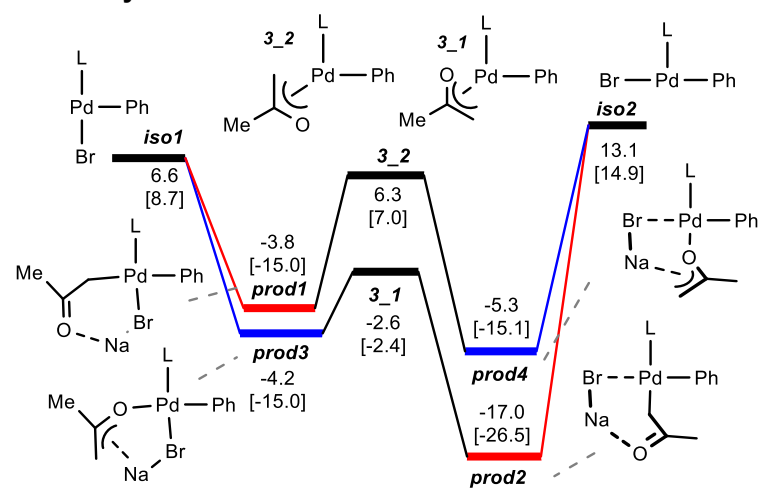

$\mathrm{NaBr}$

Zero-point correction=

Thermal correction to Energy=

Thermal correction to Enthalpy=

0.000668 (Hartree/Particle)

Sum of electronic and zero-point Energies $=\quad-175.537719$

Sum of electronic and thermal Energies $=\quad-175.534929$

Sum of electronic and thermal Enthalpies $=\quad-175.533985$

Sum of electronic and thermal Free Energies $=-175.561368$ Electronic energy (M06): -175.5098098

$\begin{array}{llll}\mathrm{Na} & 0.00000000 & 0.00000000 & -1.93379200\end{array}$

$\begin{array}{llll}\mathrm{Br} & 0.00000000 & 0.00000000 & 0.60776300\end{array}$

\section{Ketone enolate}

Zero-point correction=

Thermal correction to Energy=

Thermal correction to Enthalpy= 0.078174 (Hartree/Particle)

Thermal correction to Gibbs Free Energy $=\quad 0.048848$

Sum of electronic and zero-point Energies $=\quad-353.145596$

Sum of electronic and thermal Energies $=\quad-353.139670$ 


\begin{tabular}{|c|c|c|c|c|}
\hline \multicolumn{5}{|c|}{ Sum of electronic and thermal Enthalpies $=$} \\
\hline & tronic and the & mal Free Ener & gies $=$ & \\
\hline \multicolumn{5}{|c|}{ Electronic energy (M06): -354.7863109} \\
\hline $\mathrm{Na}$ & 1.87185400 & -0.14911100 & -0.37 & $9436 c$ \\
\hline C & -0.09279400 & 1.34739200 & 0.2 & 1426 \\
\hline $\mathrm{H}$ & 0.71761300 & 1.62560300 & 0.86 & 49950 \\
\hline $\mathrm{H}$ & -0.72010300 & 2.15178500 & -0.13 & 43270 \\
\hline 0 & 0.16125600 & -0.94679800 & & 4013 \\
\hline C & -0.53324600 & 0.04363200 & 0.19 & 8243 \\
\hline C & -1.89972600 & -0.28 & -0 . & \\
\hline $\mathrm{H}$ & -2.51389700 & -0.68902700 & & \\
\hline $\mathrm{H}$ & -2.40175200 & 0.57147600 & & 841 \\
\hline $\mathrm{H}$ & -1.80771600 & -1.06550200 & & \\
\hline
\end{tabular}

\section{Prod1}

Zero-point correction $=$

Thermal correction to Enthalpy=

0.541165 (Hartree/Particle) 0.575997 0.576941

Thermal correction to Gibbs Free Energy $=\quad 0.476768$

Sum of electronic and zero-point Energies $=\quad-1540.841339$

Sum of electronic and thermal Energies $=\quad-1540.806507$

Sum of electronic and thermal Enthalpies $=\quad-1540.805563$

Sum of electronic and thermal Free Energies $=-1540.905735$

Electronic energy (M06): -1540.939437

$\begin{array}{crrr}\mathrm{Br} & -2.74002500 & -0.77696000 & -1.87587400 \\ \mathrm{P} & 1.79783800 & 0.22731400 & -0.07464600 \\ \mathrm{C} & -1.41214200 & 1.36683600 & 0.30443100 \\ \mathrm{C} & -1.68656700 & 1.47193600 & 1.68486300 \\ \mathrm{C} & -2.08532600 & 2.26112100 & -0.54909900 \\ \mathrm{C} & -2.59654800 & 2.42007300 & 2.18811000 \\ \mathrm{H} & -1.19363600 & 0.81079200 & 2.39389600 \\ \mathrm{C} & -2.97141200 & 3.22027800 & -0.05116500 \\ \mathrm{H} & -1.94873600 & 2.18644600 & -1.62305100 \\ \mathrm{C} & -3.23761800 & 3.30358400 & 1.32024800 \\ \mathrm{H} & -2.77987900 & 2.47158500 & 3.25961000 \\ \mathrm{H} & -3.47082800 & 3.89535100 & -0.74280900 \\ \mathrm{H} & -3.93091700 & 4.04778200 & 1.70342400 \\ \mathrm{C} & 2.21947000 & 1.77255100 & -1.20160900 \\ \mathrm{C} & 2.88244900 & -1.26162100 & -0.75827900 \\ \mathrm{C} & 2.39083500 & 0.63076700 & 1.73973000 \\ \mathrm{Na} & -3.44874900 & -0.59966100 & 0.77061000 \\ \mathrm{Pd} & -0.53425900 & -0.42760500 & -0.22791500 \\ \mathrm{C} & -0.59745200 & -2.76546100 & -0.03767600 \\ \mathrm{H} & -1.31068700 & -2.82844700 & -0.85511900 \\ \mathrm{H} & 0.28161000 & -3.38855300 & -0.17461700 \\ \mathrm{O} & -2.25854200 & -2.21878300 & 1.58593500 \\ \mathrm{C} & -1.15272300 & -2.75558200 & 1.28125600 \\ \mathrm{C} & -0.39201800 & -3.41281500 & 2.42756100 \\ \mathrm{H} & -0.91853500 & -4.33694200 & 2.69827300 \\ \mathrm{H} & 0.63819400 & -3.67426800 & 2.17138700 \\ \mathrm{H} & -0.40128800 & -2.76682300 & 3.31159500 \\ \mathrm{C} & 2.94919400 & -2.38522100 & 0.29968100 \\ \mathrm{H} & 1.96379300 & -2.64777700 & 0.68129200 \\ \mathrm{H} & 3.37073300 & -3.28039400 & -0.17565600 \\ \mathrm{H} & 3.59840700 & -2.13628100 & 1.14221400 \\ \mathrm{C} & 2.18572500 & -1.85348200 & -2.00888600 \\ \mathrm{H} & 2.70285600 & -2.78282100 & -2.28211200 \\ \mathrm{H} & 1.13736400 & -2.09076700 & -1.82019700 \\ \mathrm{H} & 2.23274800 & -1.19209400 & -2.87385900 \\ \mathrm{C} & 4.33698500 & -0.91411900 & -1.14520900 \\ \mathrm{H} & 4.83752700 & -1.84499400 & -1.44380800 \\ \mathrm{H} & 4.39896000 & -0.23573400 & -1.99790000 \\ \mathrm{H} & 4.91376400 & -0.48904900 & -0.32041400 \\ \mathrm{C} & 2.08381700 & 1.37390200 & -2.68905200 \\ \mathrm{H} & 2.09974500 & 2.29173700 & -3.29095400 \\ \mathrm{H} & 2.90285100 & 0.74625100 & -3.04504900 \\ \mathrm{H} & 1.13497500 & 0.86594900 & -2.88958300 \\ \mathrm{C} & 3.62521900 & 2.37367900 & -0.96901300 \\ \mathrm{H} & 4.43635100 & 1.64960100 & -1.04460500 \\ \mathrm{H} & 3.79916100 & 3.13853400 & -1.73755700\end{array}$

$\begin{array}{lrrr}\mathrm{H} & 3.70376400 & 2.87553300 & -0.00134800 \\ \mathrm{C} & 1.19195800 & 2.90380700 & -0.98864900 \\ \mathrm{H} & 0.20985600 & 2.61718400 & -1.35623700 \\ \mathrm{H} & 1.08128400 & 3.21826600 & 0.04784300 \\ \mathrm{H} & 1.52706600 & 3.77596800 & -1.56617900 \\ \mathrm{C} & 1.72423100 & -0.38729800 & 2.69211600 \\ \mathrm{H} & 2.08197000 & -1.40648000 & 2.54527100 \\ \mathrm{H} & 1.95345700 & -0.10383500 & 3.72797400 \\ \mathrm{H} & 0.63770200 & -0.39565800 & 2.57342500 \\ \mathrm{C} & 1.88948500 & 2.03008400 & 2.16358600 \\ \mathrm{H} & 2.42390600 & 2.83953600 & 1.66139000 \\ \mathrm{H} & 0.81874100 & 2.16072000 & 1.99330700 \\ \mathrm{H} & 2.07126000 & 2.14603800 & 3.24021500 \\ \mathrm{C} & 3.91742600 & 0.59056800 & 1.96763100 \\ \mathrm{H} & 4.34352900 & -0.40241700 & 1.81269000 \\ \mathrm{H} & 4.45954500 & 1.29831800 & 1.33708100 \\ \mathrm{H} & 4.11726400 & 0.86645800 & 3.01188100\end{array}$

\section{Prod2}

Zero-point correction=

Thermal correction to Energy=

Thermal correction to Enthalpy=

0.540791 (Hartree/Particle)

Thermal correction to Gibbs Free Energy $=\quad 0.473896$

Sum of electronic and zero-point Energies $=\quad-1540.862585$

Sum of electronic and thermal Energies $=\quad-1540.827515$

Sum of electronic and thermal Enthalpies $=\quad-1540.826571$

Sum of electronic and thermal Free Energies $=-1540.929479$

Electronic energy (M06): -1540.957644

\begin{tabular}{lrrr}
$\mathrm{Br}$ & 0.68275700 & -3.13907600 & -0.34823100 \\
$\mathrm{Na}$ & 2.80607400 & -2.80595000 & 1.21658800 \\
$\mathrm{Pd}$ & 0.80676800 & -0.22864600 & -0.31307900 \\
$\mathrm{C}$ & 2.84756600 & -0.51471800 & -1.00095700 \\
$\mathrm{H}$ & 2.78118600 & -1.55450100 & -1.32478900 \\
$\mathrm{H}$ & 2.94643500 & 0.16600700 & -1.84718200 \\
$\mathrm{O}$ & 4.15110100 & -1.15572400 & 0.90151100 \\
$\mathrm{C}$ & 3.86884400 & -0.30442800 & 0.02362100 \\
$\mathrm{C}$ & 4.68871800 & 0.97119800 & 0.00660400 \\
$\mathrm{H}$ & 5.60286800 & 0.76949900 & -0.56962000 \\
$\mathrm{H}$ & 4.16530900 & 1.80288100 & -0.46689500 \\
$\mathrm{H}$ & 4.98922800 & 1.24003700 & 1.02279100 \\
$\mathrm{C}$ & 1.38989700 & 1.68474100 & -0.15096900 \\
$\mathrm{C}$ & 1.66239100 & 2.22934800 & 1.11373800 \\
$\mathrm{C}$ & 1.58605200 & 2.49681400 & -1.27952800 \\
$\mathrm{C}$ & 2.08313600 & 3.55805900 & 1.24906300 \\
$\mathrm{H}$ & 1.55073300 & 1.61890400 & 2.00677000 \\
$\mathrm{C}$ & 2.00364900 & 3.82562300 & -1.14449400 \\
$\mathrm{H}$ & 1.40500600 & 2.09906400 & -2.27508800 \\
$\mathrm{C}$ & 2.25164600 & 4.36358900 & 0.12113600 \\
$\mathrm{H}$ & 2.28393300 & 3.95928700 & 2.24028800 \\
$\mathrm{H}$ & 2.13753300 & 4.43951900 & -2.03283400 \\
$\mathrm{H}$ & 2.57923400 & 5.39464600 & 0.22553600 \\
$\mathrm{P}$ & -1.79807600 & 0.20407400 & 0.06351000 \\
$\mathrm{C}$ & -2.51205100 & -1.02183600 & 1.42408800 \\
$\mathrm{C}$ & -2.37720000 & 2.00470500 & 0.60701700 \\
$\mathrm{C}$ & -2.65829400 & -0.19056600 & -1.64015400 \\
$\mathrm{C}$ & -3.88382700 & -0.65430000 & 2.02951200 \\
$\mathrm{H}$ & -4.15713300 & -1.43620400 & 2.75107400 \\
$\mathrm{H}$ & -3.89068400 & 0.29233700 & 2.57131700 \\
$\mathrm{H}$ & -4.67424500 & -0.62598500 & 1.27386000 \\
$\mathrm{C}$ & -2.65607900 & -2.46132400 & 0.88189000 \\
$\mathrm{H}$ & -1.73911200 & -2.83817500 & 0.43166600 \\
$\mathrm{H}$ & -2.89418600 & -3.11564300 & 1.73180200 \\
$\mathrm{H}$ & -3.47481500 & -2.56332100 & 0.16655700 \\
$\mathrm{C}$ & -1.45362500 & -1.08251200 & 2.55204400 \\
$\mathrm{H}$ & -1.79876000 & -1.78194400 & 3.32572200 \\
$\mathrm{H}$ & -0.49897100 & -1.45093400 & 2.16388800 \\
$\mathrm{H}$ & -1.27784500 & -0.11850500 & 3.03381600 \\
$\mathrm{C}$ & -2.40192700 & 0.97229500 & -2.62526900 \\
$\mathrm{H}$ & -3.01345400 & 1.85147400 & -2.41012400 \\
$\mathrm{H}$ & -1.34880200 & 1.27181200 & -2.64518700 \\
& & & \\
\hline & & & \\
$\mathrm{H}$ & & & \\
$\mathrm{H}$ & & & \\
$\mathrm{H}$ & & &
\end{tabular}




$\begin{array}{lrrr}\mathrm{H} & -2.66843500 & 0.63425700 & -3.63523700 \\ \mathrm{C} & -1.96661300 & -1.42827100 & -2.25425400 \\ \mathrm{H} & -2.07794700 & -2.33277600 & -1.65767400 \\ \mathrm{H} & -2.41181000 & -1.62131700 & -3.23998800 \\ \mathrm{H} & -0.89482500 & -1.25829000 & -2.39448900 \\ \mathrm{C} & -4.18009300 & -0.44558100 & -1.58776800 \\ \mathrm{H} & -4.54156500 & -0.60477600 & -2.61303800 \\ \mathrm{H} & -4.43989800 & -1.34001300 & -1.01820500 \\ \mathrm{H} & -4.74019500 & 0.39481600 & -1.17383900 \\ \mathrm{C} & -3.89237400 & 2.28199900 & 0.47248300 \\ \mathrm{H} & -4.10484800 & 3.25330200 & 0.94014800 \\ \mathrm{H} & -4.19693900 & 2.36521800 & -0.57433400 \\ \mathrm{H} & -4.53042600 & 1.54321100 & 0.95609500 \\ \mathrm{C} & -1.94487700 & 2.24229400 & 2.07285100 \\ \mathrm{H} & -2.12558600 & 3.29708400 & 2.31896000 \\ \mathrm{H} & -2.50155900 & 1.64556800 & 2.79672500 \\ \mathrm{H} & -0.87610500 & 2.05748200 & 2.21120600 \\ \mathrm{C} & -1.65697400 & 3.10158500 & -0.21129400 \\ \mathrm{H} & -0.58354900 & 3.09442500 & -0.04840700 \\ \mathrm{H} & -1.84021700 & 3.04076500 & -1.28284800 \\ \mathrm{H} & -2.03962800 & 4.07404400 & 0.12790700\end{array}$

Prod3

Zero-point correction $=$ 0.540957 (Hartree/Particle)

Thermal correction to Energy=

Thermal correction to Enthalpy= 0.575694

Thermal correction to Gibbs Free Energy=
Sum of electronic and zero-point Energies= 0.576638

Sum of electronic and thermal Energies=

0.475808

$-1540.845612$

$-1540.810875$

Sum of electronic and thermal Enthalpies $=\quad-1540.809931$

Sum of electronic and thermal Free Energies $=-1540.910761$

Electronic energy (M06): -1540.939168

$\begin{array}{lrrr}\mathrm{Br} & -3.25887500 & 0.54112100 & 0.91929100 \\ \mathrm{P} & 1.70873500 & -0.19855200 & 0.12372900 \\ \mathrm{C} & -0.71994400 & 1.95130900 & -0.40277300 \\ \mathrm{C} & -0.83289900 & 2.94371200 & 0.57598800 \\ \mathrm{C} & -0.76558500 & 2.31171200 & -1.75526000 \\ \mathrm{C} & -0.96165900 & 4.28518000 & 0.20411300 \\ \mathrm{H} & -0.84920100 & 2.67801400 & 1.62834200 \\ \mathrm{C} & -0.90323500 & 3.65628800 & -2.12352700 \\ \mathrm{H} & -0.69698100 & 1.55238500 & -2.53085700 \\ \mathrm{C} & -0.99195500 & 4.64762700 & -1.14492300 \\ \mathrm{H} & -1.05170600 & 5.04749900 & 0.97490500 \\ \mathrm{H} & -0.93930700 & 3.92263000 & -3.17764800 \\ \mathrm{H} & -1.09536400 & 5.69132900 & -1.43011600 \\ \mathrm{C} & 2.90415400 & 1.19534500 & -0.57861000 \\ \mathrm{C} & 2.25349400 & -1.87779900 & -0.72371600 \\ \mathrm{C} & 2.01612600 & -0.33864400 & 2.04585500 \\ \mathrm{Na} & -3.41563700 & -2.08045900 & 0.60358200 \\ \mathrm{Pd} & -0.74555900 & -0.00745800 & -0.03319300 \\ \mathrm{C} & -1.76907200 & -2.89270700 & -1.06184700 \\ \mathrm{C} & -1.39971100 & -4.36415700 & -1.18310000 \\ \mathrm{H} & -0.33704900 & -4.46664000 & -1.42988000 \\ \mathrm{H} & -1.54918200 & -4.88085700 & -0.22630900 \\ \mathrm{H} & -1.98288000 & -4.87223000 & -1.95713500 \\ \mathrm{C} & 1.75164500 & -3.08092800 & 0.10512100 \\ \mathrm{H} & 0.68208100 & -3.01444900 & 0.30954200 \\ \mathrm{H} & 1.93054100 & -3.99040100 & -0.48490700 \\ \mathrm{H} & 2.30005700 & -3.20282300 & 1.04164400 \\ \mathrm{C} & 1.54652400 & -1.94141400 & -2.09846200 \\ \mathrm{H} & 1.76082400 & -2.91594500 & -2.55766100 \\ \mathrm{H} & 0.46324400 & -1.84555400 & -1.99873800 \\ \mathrm{H} & 1.89257200 & -1.17425600 & -2.79302100 \\ \mathrm{C} & 3.76991300 & -2.07801300 & -0.93352000 \\ \mathrm{H} & 3.91910000 & -3.07293500 & -1.37397000 \\ \mathrm{H} & 4.21511200 & -1.35779800 & -1.62192200 \\ \mathrm{H} & 4.33180800 & -2.05628000 & 0.00425900 \\ \mathrm{C} & 2.90462800 & 1.12416700 & -2.12279600 \\ \mathrm{H} & 3.42709400 & 2.01061900 & -2.50498600 \\ \mathrm{H} & & & \\ & 3.42351900 & 0.25031000 & -2.51972900\end{array}$

$\begin{array}{lll}1.88831500 & 1.14607800 & -2.52686600\end{array}$

$\begin{array}{llll}4.35916800 & 1.08448800 & -0.06344800\end{array}$

$\begin{array}{llll}4.80491400 & 0.09734600 & -0.17841700\end{array}$

$4.97590500 \quad 1.78974000-0.63653800$

$\begin{array}{llll}4.44037500 & 1.37767900 & 0.98671200\end{array}$

$2.42434300 \quad 2.62653400 \quad-0.23643400$

$\begin{array}{llll}1.53325400 & 2.90066600 & -0.79484400\end{array}$

$\begin{array}{lll}2.22538100 & 2.79000600 & 0.82077200\end{array}$

$3.22720200 \quad 3.31677100 \quad-0.52957300$

$0.84661500-1.13583000 \quad 2.67153400$

$\begin{array}{llll}0.75188300 & -2.14832700 & 2.28032700\end{array}$

$1.01860500-1.20696600 \quad 3.75420600$

$\begin{array}{lll}-0.10929600 & -0.62538400 & 2.51511600\end{array}$

$\begin{array}{lll}1.97133900 & 1.06593200 & 2.68739200\end{array}$

$\begin{array}{lll}2.85566500 & 1.66507900 & 2.45821400\end{array}$

$\begin{array}{lll}1.08040700 & 1.62748700 & 2.39345700\end{array}$

$\begin{array}{lll}1.93910500 & 0.94279800 & 3.77774600\end{array}$

$\begin{array}{llll}3.35032900 & -1.00159500 & 2.45373000\end{array}$

$\begin{array}{llll}3.42206200 & -2.04528900 & 2.14210700\end{array}$

$\begin{array}{llll}4.22298300 & -0.47025000 & 2.06939900\end{array}$

$\begin{array}{lll}3.42025800 & -0.98948400 & 3.54986200\end{array}$

$-1.25725100-2.26814000-0.00401700$

$-2.63729100-2.32969600-1.95353100$

$-3.03203000-2.90325800-2.78563300$

$-2.85550000-1.26410900-1.93008200$

Prod4

Zero-point correction= 0.540393 (Hartree/Particle)

Thermal correction to Energy=

Thermal correction to Enthalpy= 0.575463

marrection to Gibbs Free Energy $=\quad 0.473979$

Sum of electronic and zero-point Energies $=\quad-1540.849119$

Sum of electronic and thermal Energies $=\quad-1540.814049$

Sum of electronic and thermal Enthalpies $=\quad-1540.813105$

Sum of electronic and thermal Free Energies $=-1540.915533$

Electronic energy (M06): -1540.939033

$\begin{array}{llll}\mathrm{Br} & -0.62793000 & -3.16430100 & 0.28155200\end{array}$

$\mathrm{Na} \quad-3.20900700 \quad-2.66231600 \quad-0.06413900$

$\begin{array}{llll}\mathrm{Pd} & -0.65264100 & -0.26407800 & 0.13777600\end{array}$

$\begin{array}{llll}\text { C } & -3.75838600 & -0.19888700 & -0.37581200\end{array}$

$\begin{array}{llll}\text { C } & -4.83960700 & 0.64991800 & 0.26146100\end{array}$

$\begin{array}{llll}\mathrm{H} & -5.18234800 & 0.19601800 & 1.19917000\end{array}$

$\begin{array}{llll}\mathrm{H} & -4.41424700 & 1.62723200 & 0.51654400\end{array}$

H $\quad-5.69793900 \quad 0.79837100 \quad-0.40111700$

$\begin{array}{llll}\text { C } & -1.25147600 & 1.63934000 & 0.14406900\end{array}$

C $\quad \begin{array}{llll}\text { C } & -1.65065300 & 2.26163300 & -1.04324700\end{array}$

C $\quad-1.47418300 \quad 2.28436900 \quad 1.36637200$

C $\quad-2.22780300 \quad 3.53795900-1.01049300$

$\begin{array}{llll}\mathrm{H} & -1.53053600 & 1.75737200 & -1.99794200\end{array}$

$\begin{array}{llll}-2.04852400 & 3.55960100 & 1.39534900\end{array}$

$\begin{array}{llll}\text { H } & -1.21057400 & 1.79682000 & 2.30086400\end{array}$

$\begin{array}{llll}-2.41945300 & 4.19467200 & 0.20652500\end{array}$

H $\quad-2.52968200 \quad 4.01350100 \quad-1.94116100$

$\begin{array}{llll}H & -2.21119300 & 4.05345100 & 2.35092000\end{array}$

$\begin{array}{llll}\mathrm{H} & -2.86350600 & 5.18646300 & 0.23046600\end{array}$

$\begin{array}{llll}\mathrm{P} & 1.74941300 & 0.22712000 & -0.05479200\end{array}$

C $\quad 2.51155700 \quad-1.00883700 \quad-1.38441400$

$\begin{array}{llll}\text { C } & 2.30379400 & 2.03301300 & -0.61258800\end{array}$

C $\quad 2.55575200 \quad-0.12700400 \quad 1.68246000$

$\begin{array}{rrr}2.55575200 & -0.12700400 & 1.68246000 \\ 3.88713500 & -0.61472300 & -1.96533100\end{array}$

$\begin{array}{lll}4.18484600 & -1.39919300 & -2.67346700\end{array}$

$\begin{array}{lll}3.88708600 & 0.32539000 & -2.51751600\end{array}$

$\begin{array}{lll}4.66431800 & -0.56799400 & -1.19721600\end{array}$

$\begin{array}{lll}2.68834400 & -2.43298900 & -0.81312400\end{array}$

$\begin{array}{lll}1.77521000 & -2.83136300 & -0.37593500\end{array}$

$\begin{array}{lll}2.95991100 & -3.09206900 & -1.64849600\end{array}$

$3.49746800 \quad-2.49845200 \quad-0.08254200$

$\begin{array}{lll}1.48237000 & -1.11381400 & -2.53587600\end{array}$

$\begin{array}{lll}1.87596400 & -1.80302600 & -3.29492400\end{array}$

$\begin{array}{llll}0.53209000 & -1.51890700 & -2.17637600\end{array}$ 


$\begin{array}{lrrr}\mathrm{H} & 1.28567000 & -0.15909300 & -3.02811600 \\ \mathrm{C} & 2.24841100 & 1.04437000 & 2.64195900 \\ \mathrm{H} & 2.84556300 & 1.93374400 & 2.42831000 \\ \mathrm{H} & 1.18935300 & 1.32045400 & 2.63194900 \\ \mathrm{H} & 2.49710100 & 0.72677000 & 3.66278100 \\ \mathrm{C} & 1.87999900 & -1.37543600 & 2.29194400 \\ \mathrm{H} & 2.03122600 & -2.28218600 & 1.70809800 \\ \mathrm{H} & 2.30684100 & -1.54379300 & 3.28997100 \\ \mathrm{H} & 0.80103600 & -1.23631800 & 2.40287300 \\ \mathrm{C} & 4.08445500 & -0.34800200 & 1.67149100 \\ \mathrm{H} & 4.41394500 & -0.51391100 & 2.70604600 \\ \mathrm{H} & 4.38223200 & -1.22850900 & 1.09919200 \\ \mathrm{H} & 4.63983600 & 0.51050100 & 1.29048900 \\ \mathrm{C} & 3.81871300 & 2.30311600 & -0.44086600 \\ \mathrm{H} & 4.05588500 & 3.24083200 & -0.96093500 \\ \mathrm{H} & 4.08222000 & 2.45209600 & 0.60990100 \\ \mathrm{H} & 4.46869200 & 1.53241400 & -0.85046700 \\ \mathrm{C} & 1.90876700 & 2.24291800 & -2.09316000 \\ \mathrm{H} & 2.08340000 & 3.29675200 & -2.34692500 \\ \mathrm{H} & 2.49114700 & 1.64431600 & -2.79450500 \\ \mathrm{H} & 0.84683000 & 2.04251400 & -2.25769900 \\ \mathrm{C} & 1.58273100 & 3.16192900 & 0.16521200 \\ \mathrm{H} & 0.53638300 & 3.24691200 & -0.11104500 \\ \mathrm{H} & 1.64300100 & 3.06481600 & 1.24724100 \\ \mathrm{H} & 2.07477400 & 4.10616800 & -0.10530700 \\ \mathrm{C} & -3.85566900 & -0.62432600 & -1.67041200 \\ \mathrm{H} & -4.69789600 & -0.34326600 & -2.29323200 \\ \mathrm{H} & -3.01117600 & -1.10505400 & -2.16364500 \\ \mathrm{O} & -2.77545400 & -0.54823400 & 0.44875200\end{array}$

n3_1

Zero-point correction= 0.538615 (Hartree/Particle)

Thermal correction to Energy= $\quad 0.569462$

Thermal correction to Enthalpy $=\quad 0.570406$

Thermal correction to Gibbs Free Energy $=\quad 0.479429$

Sum of electronic and zero-point Energies $=\quad-1365.286967$

Sum of electronic and thermal Energies $=\quad-1365.256120$

Sum of electronic and thermal Enthalpies $=\quad-1365.255176$

Sum of electronic and thermal Free Energies $=-1365.346153$

Electronic energy (M06): -1365.407437

$\begin{array}{llll}\mathrm{P} & 1.30490800 & -0.33648400 & -0.05602000\end{array}$

$\begin{array}{llll}\text { C } & -2.21917700 & -0.61968400 & 0.04449200\end{array}$

C $\quad-2.90165500 \quad-0.78928800-1.17146400$

$\begin{array}{llll}\text { C } & -2.59265000 & -1.42641800 & 1.13008400\end{array}$

C $\quad-3.90018300-1.76053700-1.30652600$

$\mathrm{H} \quad-2.65315700 \quad-0.16609200 \quad-2.02747200$

C $\quad-3.59082900 \quad-2.39884800 \quad 0.99468500$

H $\quad-2.11442700 \quad-1.29793700 \quad 2.09805400$

C $\quad-4.24567700 \quad-2.57399200 \quad-0.22534900$

$\mathrm{H} \quad-4.40989100-1.87684900-2.26085100$

$\mathrm{H} \quad-3.85847400 \quad-3.01528400 \quad 1.85051000$

$\mathrm{H} \quad-5.02163700 \quad-3.32794800 \quad-0.33008400$

C $\quad 1.75052100-1.16703000 \quad 1.64850100$

$\begin{array}{llll}\text { C } & 2.62839000 & 1.05510000 & -0.42842000\end{array}$

C $\quad 1.44360300-1.68349600-1.45668200$

$\begin{array}{llll}\mathrm{Pd} & -0.84843600 & 0.84468200 & 0.23618300\end{array}$

C $\quad \begin{array}{llll}-1.52527800 & 3.11228600 & 0.16016000\end{array}$

$\begin{array}{llll}\text { C } & 1.22097900 & -0.24587800 & 2.77439600\end{array}$

$\begin{array}{llll}\mathrm{H} & 1.39616000 & -0.73664600 & 3.74130100\end{array}$

$\begin{array}{llll}\mathrm{H} & 1.71180700 & 0.72712300 & 2.80553700\end{array}$

H $\quad 0.14463500 \quad-0.07241700 \quad 2.67197100$

$\begin{array}{llll}\text { C } & 0.99374200 & -2.50628300 & 1.79520600\end{array}$

$\begin{array}{llll}\mathrm{H} & -0.07551100 & -2.40656800 & 1.59135700\end{array}$

$\mathrm{H} \quad \begin{array}{llll}1.39936100 & -3.29300000 & 1.15494300\end{array}$

$\begin{array}{llll}\mathrm{H} & 1.10306900 & -2.84960300 & 2.83254700\end{array}$

$\begin{array}{llll}\text { C } & 3.25029300 & -1.44026400 & 1.88734200\end{array}$

$\mathrm{H} \quad 3.84489600 \quad-0.52518400 \quad 1.92506900$

$\mathrm{H} \quad 3.36490800 \quad-1.93974400 \quad 2.85900900$

$\mathrm{H} \quad 3.68458700 \quad-2.09891000 \quad 1.13092500$

$\begin{array}{llll}\text { C } & 2.82932300 & 1.93702900 & 0.82581000\end{array}$

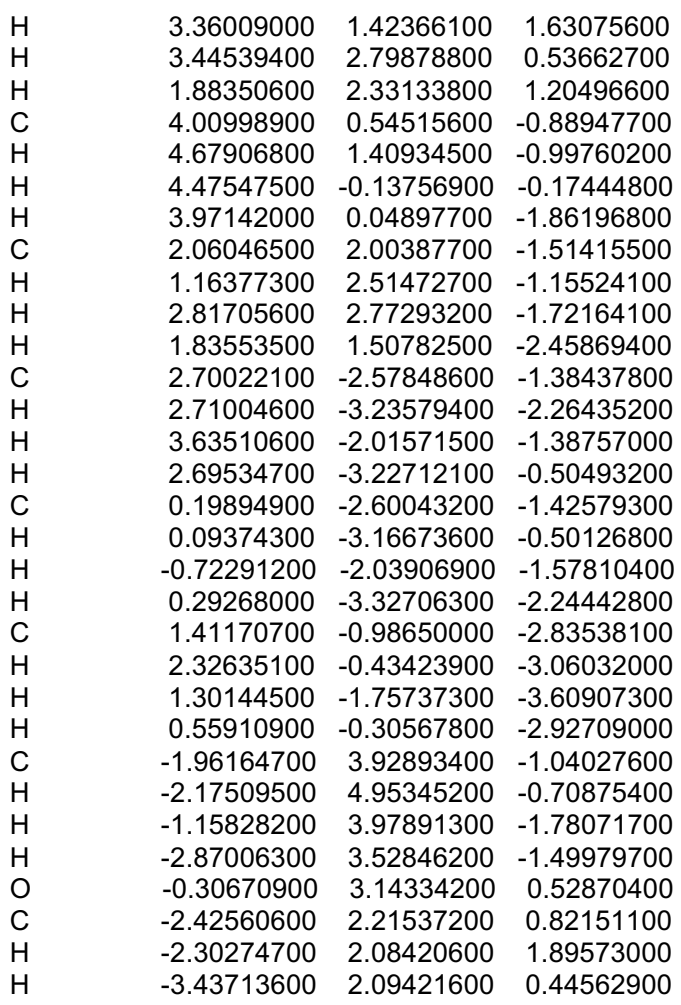

n3_2

Zero-point correction= 0.538317 (Hartree/Particle)

Thermal correction to Energy= $\quad 0.569311$

Thermal correction to Enthalpy= $\quad 0.570255$

Thermal correction to Gibbs Free Energy $=\quad 0.478711$

Sum of electronic and zero-point Energies $=\quad-1365.271285$

Sum of electronic and thermal Energies $=\quad-1365.240291$

Sum of electronic and thermal Enthalpies $=\quad-1365.239347$

Sum of electronic and thermal Free Energies $=-1365.330891$

Electronic energy (M06): -1365.392403

$\begin{array}{llll}P & 1.26248800 & -0.32891800 & -0.04597500\end{array}$

C $\quad-2.11809900 \quad-0.71516500 \quad 0.04097600$

C $\quad-2.81101600 \quad-0.90472200-1.16335900$

C $\quad-2.51946800 \quad-1.44447200 \quad 1.16757700$

C $\quad-3.86261800-1.82451300-1.24472600$

$\begin{array}{llll}\mathrm{H} & -2.53736500 & -0.33594500 & -2.04889200\end{array}$

C $\quad-3.57043500 \quad-2.36593600 \quad 1.08529600$

H $\quad-2.03035700 \quad-1.29020500 \quad 2.12639300$

C $\quad-4.24160800 \quad-2.56379100 \quad-0.12221700$

$\mathrm{H} \quad-4.38777000-1.95819500 \quad-2.18831900$

$\begin{array}{llll}\mathrm{H} & -3.86689000 & -2.92322000 & 1.97163200\end{array}$

H $\quad-5.05804000 \quad-3.27868300 \quad-0.18587100$

C $\quad \begin{array}{llll}1.88192800 & -0.90359400 & 1.71160400\end{array}$

$\begin{array}{llll}\text { C } & 2.52455300 & 1.00684400 & -0.73906300\end{array}$

C $\quad \begin{array}{llll}\text { C } & 1.33158400 & -1.86586900 & -1.25478500\end{array}$

$\begin{array}{llll}\text { Pd } & -0.81579600 & 0.82213400 & 0.21830100\end{array}$

$\begin{array}{llll}\text { C } & -0.43472900 & 3.20645000 & 0.86006500\end{array}$

$\begin{array}{llll}\mathrm{H} & -0.34485300 & 2.77656300 & 1.85377000\end{array}$

$\begin{array}{llll}\mathrm{H} & 0.29551000 & 3.95885200 & 0.57872100\end{array}$

$\begin{array}{llll}\text { O } & -2.47013100 & 2.14480000 & 0.44974100\end{array}$

C $\quad-1.65020000 \quad 3.11668700 \quad 0.18398600$

$\begin{array}{llll}\text { C } & 1.44518000 & 0.16111800 & 2.74511800\end{array}$

$\begin{array}{llll}\mathrm{H} & 1.72509800 & -0.18836100 & 3.74781600\end{array}$

$\begin{array}{llll}\mathrm{H} & 1.91837400 & 1.13142100 & 2.59213800\end{array}$

$\begin{array}{llll}\mathrm{H} & 0.36046300 & 0.30783000 & 2.72710700\end{array}$

$\begin{array}{llll}\text { C } & 1.17142600 & -2.21451200 & 2.11544500\end{array}$

H $\quad 0.08755500 \quad-2.16007000 \quad 1.98608100$

H $\quad 1.54601700-3.08159400 \quad 1.56709400$ 


$\begin{array}{lrrr}\mathrm{H} & 1.36999500 & -2.39840600 & 3.17948000 \\ \mathrm{C} & 3.40324300 & -1.13338700 & 1.83577500 \\ \mathrm{H} & 3.98546500 & -0.22143100 & 1.69016500 \\ \mathrm{H} & 3.61874700 & -1.49198300 & 2.85127600 \\ \mathrm{H} & 3.77438400 & -1.88962600 & 1.14024800 \\ \mathrm{C} & 2.88420400 & 2.03172700 & 0.35941600 \\ \mathrm{H} & 3.53673100 & 1.61936500 & 1.13180900 \\ \mathrm{H} & 3.43206700 & 2.85823800 & -0.11215200 \\ \mathrm{H} & 1.99743100 & 2.45455300 & 0.83228400 \\ \mathrm{C} & 3.85183200 & 0.45210300 & -1.29913600 \\ \mathrm{H} & 4.48720600 & 1.30265600 & -1.57986500 \\ \mathrm{H} & 4.40906700 & -0.13864800 & -0.56766900 \\ \mathrm{H} & 3.71684400 & -0.15010500 & -2.19991300 \\ \mathrm{C} & 1.81151400 & 1.80282900 & -1.85929800 \\ \mathrm{H} & 0.90309800 & 2.28309200 & -1.48591600 \\ \mathrm{H} & 2.49015300 & 2.59079300 & -2.21306600 \\ \mathrm{H} & 1.54417800 & 1.19167600 & -2.72183100 \\ \mathrm{C} & 2.64358200 & -2.67862000 & -1.18267400 \\ \mathrm{H} & 2.61573600 & -3.44287900 & -1.97066200 \\ \mathrm{H} & 3.54496600 & -2.08579200 & -1.33840000 \\ \mathrm{H} & 2.74156500 & -3.20859400 & -0.23179400 \\ \mathrm{C} & 0.17019200 & -2.84950100 & -0.98140000 \\ \mathrm{H} & 0.17751100 & -3.27193700 & 0.02218400 \\ \mathrm{H} & -0.80159900 & -2.38894800 & -1.14601000 \\ \mathrm{H} & 0.27376600 & -3.68507700 & -1.68675300 \\ \mathrm{C} & 1.12018300 & -1.36459200 & -2.70123400 \\ \mathrm{H} & 1.97654700 & -0.81554600 & -3.09766000 \\ \mathrm{H} & 0.96774800 & -2.23651300 & -3.34998500 \\ \mathrm{H} & 0.22845100 & -0.73446900 & -2.78356400 \\ \mathrm{C} & -2.02022500 & 4.05151900 & -0.94906900 \\ \mathrm{H} & -2.92679100 & 4.60084400 & -0.66867800 \\ \mathrm{H} & -1.23007400 & 4.77552600 & -1.16919100 \\ \mathrm{H} & -2.25674300 & 3.47841400 & -1.85197300\end{array}$

Reactivity of ester enolate

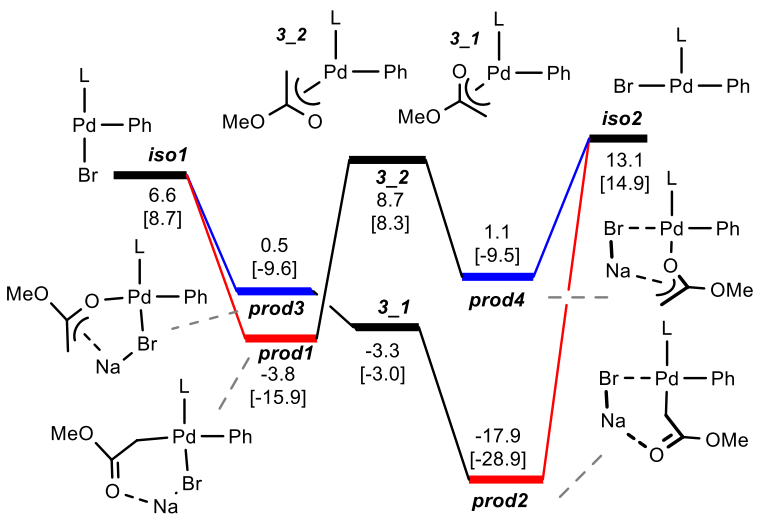

Ester_enolate

Zero-point correction=

Thermal correction to Energy=

Thermal correction to Enthalpy=

0.078534 (Hartree/Particle) 0.085648 0.086593

Thermal correction to Gibbs Free Energy $=\quad 0.047244$

Sum of electronic and zero-point Energies $=\quad-430.001601$

Sum of electronic and thermal Energies $=\quad-429.994487$

Sum of electronic and thermal Enthalpies $=\quad-429.993542$

Sum of electronic and thermal Free Energies $=\quad-430.032891$

Electronic energy (M06): -430.0000454

$\begin{array}{lrrr}\mathrm{Na} & 2.08198300 & -0.65017000 & -0.40679600 \\ \mathrm{C} & 0.85911100 & 1.39364700 & 0.05959000 \\ \mathrm{H} & 1.62415000 & 1.48500000 & 0.83270300 \\ \mathrm{H} & 0.50580700 & 2.33327200 & -0.35052700 \\ \mathrm{O} & 0.20584200 & -0.80311100 & 0.71552300 \\ \mathrm{C} & -0.06694900 & 0.35036000 & 0.25356600 \\ \mathrm{O} & -1.33838800 & 0.59867100 & -0.19831600 \\ \mathrm{C} & -2.22288800 & -0.51815000 & -0.22020800 \\ \mathrm{H} & -2.36925000 & -0.93463600 & 0.78077700\end{array}$

$\mathrm{H}$

$\mathrm{H}$

$-3.16954300-0.13611500-0.60936100$

$-1.84825000-1.31528000-0.87418400$

Prod1

Zero-point correction= 0.546931 (Hartree/Particle)

Thermal correction to Energy=

Thermal correction to Enthalpy=

0.582461

0.583405

Thermal correction to Gibbs Free Energy $=\quad 0.481563$

Sum of electronic and zero-point Energies $=\quad-1616.064308$

Sum of electronic and thermal Energies $=\quad-1616.028779$

Sum of electronic and thermal Enthalpies $=\quad-1616.027835$

Sum of electronic and thermal Free Energies $=-1616.129676$

Electronic energy (M06): -1616.15952

\begin{tabular}{|c|c|c|c|}
\hline$B r$ & 2.67311300 & -0.16282100 & -2.03574200 \\
\hline$P$ & -1.86386000 & -0.18938000 & 0.00466700 \\
\hline C & 1.26322900 & -1.44883900 & 0.64279600 \\
\hline C & 1.76159100 & -1.10816100 & 1.92146800 \\
\hline C & 1.66700500 & -2.68609600 & 0.11424400 \\
\hline C & 2.63527500 & -1.95364100 & 2.62772200 \\
\hline $\mathrm{H}$ & 1.44406800 & -0.17892400 & 2.39729400 \\
\hline $\mathrm{C}$ & 2.51241000 & -3.54482400 & 0.82449400 \\
\hline $\mathrm{H}$ & 1.35841300 & -2.97388000 & -0.88632100 \\
\hline C & 3.00928500 & -3.18229100 & 2.08037400 \\
\hline $\mathrm{H}$ & 2.99200500 & -1.66094700 & 3.61393100 \\
\hline $\mathrm{H}$ & 2.80255600 & -4.49573900 & 0.38238300 \\
\hline $\mathrm{H}$ & 3.67169500 & -3.84980100 & 2.62521900 \\
\hline & -2.28835600 & -1.41324300 & -1.46724600 \\
\hline C & -2.93369200 & 1.43675600 & -0.25246200 \\
\hline C & -2.49929500 & -1.01021600 & 1.66732100 \\
\hline $\mathrm{Na}$ & 3.49376700 & 0.50566900 & 0.47194500 \\
\hline $\mathrm{Pd}$ & 0.51242400 & 0.21420700 & -0.33941200 \\
\hline C & 0.69439200 & 2.38804700 & -0.93559800 \\
\hline $\mathrm{H}$ & 1.34742700 & 2.23263400 & -1.79136000 \\
\hline $\mathrm{H}$ & -0.16478600 & 3.00903600 & -1.17102100 \\
\hline & 2.51985000 & 2.46151400 & 0.65463000 \\
\hline C & 1.41257100 & 2.85936100 & 0.23250300 \\
\hline $\mathrm{O}$ & 0.74077700 & 3.80287300 & 0.95066200 \\
\hline C & 1.34742600 & 4.22876700 & 2.17331300 \\
\hline $\mathrm{H}$ & 2.33404000 & 4.66405000 & 1.99112100 \\
\hline $\mathrm{H}$ & 0.67376600 & 4.98118200 & 2.58693200 \\
\hline $\mathrm{H}$ & 1.45372200 & 3.39387300 & 2.87393000 \\
\hline C & -1.45300100 & -1.00628100 & -2.70447900 \\
\hline $\mathrm{H}$ & -1.65991600 & -1.72406000 & -3.50990100 \\
\hline $\mathrm{H}$ & -1.69804600 & -0.01263600 & -3.08091700 \\
\hline $\mathrm{H}$ & -0.37922900 & -1.03138400 & -2.49647700 \\
\hline C & -1.85618000 & -2.84967000 & -1.10100500 \\
\hline $\mathrm{H}$ & -0.82580100 & -2.89386200 & -0.74639100 \\
\hline $\mathrm{H}$ & -2.50456300 & -3.31116800 & -0.35253200 \\
\hline $\mathrm{H}$ & -1.92290400 & -3.46686200 & -2.00654100 \\
\hline C & -3.77660700 & -1.46719300 & -1.87770900 \\
\hline $\mathrm{H}$ & -4.13165000 & -0.53153900 & -2.31427600 \\
\hline $\mathrm{H}$ & -3.88803100 & -2.24005000 & -2.64997000 \\
\hline $\mathrm{H}$ & -4.43994800 & -1.73680500 & -1.05312800 \\
\hline C & -2.75563000 & 1.92087900 & -1.70981300 \\
\hline $\mathrm{H}$ & -3.30308400 & 1.30404900 & -2.42565100 \\
\hline $\mathrm{H}$ & -3.15975500 & 2.93844900 & -1.78816100 \\
\hline $\mathrm{H}$ & -1.70820800 & 1.95441400 & -2.01059500 \\
\hline C & -4.44995000 & 1.30603800 & 0.01475900 \\
\hline $\mathrm{H}$ & -4.92172600 & 2.26273100 & -0.24728200 \\
\hline $\mathrm{H}$ & -4.93415400 & 0.53284000 & -0.58418200 \\
\hline $\mathrm{H}$ & -4.67570200 & 1.12170000 & 1.06772900 \\
\hline C & -2.40021100 & 2.54963600 & 0.68037300 \\
\hline $\mathrm{H}$ & -1.31723200 & 2.64776500 & 0.65258200 \\
\hline $\mathrm{H}$ & -2.82764700 & 3.50762200 & 0.35577100 \\
\hline $\mathrm{H}$ & -2.70235800 & 2.40406700 & 1.71804800 \\
\hline C & -3.90914400 & -1.63944300 & 1.57699800 \\
\hline $\mathrm{H}$ & -4.20792600 & -1.95243100 & 2.58658500 \\
\hline $\mathrm{H}$ & -4.67578800 & -0.95693600 & 1.21014300 \\
\hline $\mathrm{H}$ & -3.92131800 & -2.53536800 & 0.95218400 \\
\hline & -1.53346400 & -2.11549300 & 2.14845800 \\
\hline
\end{tabular}




$\begin{array}{lrrr}\mathrm{H} & -1.34852000 & -2.89667700 & 1.41260000 \\ \mathrm{H} & -0.56947400 & -1.70644100 & 2.44511800 \\ \mathrm{H} & -1.98299900 & -2.59168500 & 3.03057700 \\ \mathrm{C} & -2.50826200 & 0.05136300 & 2.79068600 \\ \mathrm{H} & -3.33494400 & 0.75910500 & 2.70367200 \\ \mathrm{H} & -2.62695900 & -0.46543800 & 3.75183400 \\ \mathrm{H} & -1.57037300 & 0.61484300 & 2.83408700\end{array}$

Prod2

Zero-point correction=

$\begin{array}{lc}\text { Thermal correction to Energy= } & 0.582679 \\ \text { Thermal correction to Enthalpy= } & 0.583623 \\ \text { Thermal correction to Gibbs Free Energy= } & 0.479994 \\ \text { Sum of electronic and zero-point Energies }= & -1616.085648 \\ \text { Sum of electronic and thermal Energies }= & -1616.049916 \\ \text { Sum of electronic and thermal Enthalpies }= & -1616.04897\end{array}$

Sum of electronic and thermal Free Energies $=-1616.152601$

Electronic energy (M06): -1616.18053

$\begin{array}{llll}\mathrm{Na} & -2.04975900 & -2.57220500 & -1.38822400\end{array}$

$\begin{array}{llll}\mathrm{Pd} & -0.57947700 & -0.41079200 & 0.35274700\end{array}$

$\begin{array}{llll}\text { C } & -2.50676000 & -0.99884000 & 1.10050100\end{array}$

$\begin{array}{llll}\mathrm{H} & -2.35748600 & -2.06086000 & 1.30455400\end{array}$

$\begin{array}{llll}\mathrm{H} & -2.64311900 & -0.44026200 & 2.02616500\end{array}$

$\begin{array}{llll}\text { O } & -3.84617300 & -1.45246600 & -0.86135700\end{array}$

$\begin{array}{llll}\text { C } & -3.63353300 & -0.79558000 & 0.17667500\end{array}$

$\begin{array}{lllll}\text { O } & -4.46332400 & 0.19579400 & 0.53052300\end{array}$

$\begin{array}{lllll}\text { C } & -5.51121700 & 0.52400500 & -0.39299700\end{array}$

$\begin{array}{llll}\mathrm{H} & -6.17113200 & -0.33270000 & -0.55294200\end{array}$

$\begin{array}{llll}\mathrm{H} & -6.05557900 & 1.34678700 & 0.07098600\end{array}$

$\begin{array}{llll}\mathrm{H} & -5.08813600 & 0.84255100 & -1.34906800\end{array}$

$\begin{array}{llll}\text { C } & -1.49428600 & 1.37115600 & 0.17294900\end{array}$

C $\quad-1.87958100 \quad 1.82778200 \quad-1.09786900$

$\begin{array}{llll}\text { C } & -1.80868700 & 2.16647900 & 1.28526200\end{array}$

$\begin{array}{llll}\text { C } & -2.52795100 & 3.05950100 & -1.25472900\end{array}$

$\mathrm{H} \quad-1.66794100 \quad 1.23074000 \quad-1.98235700$

$\begin{array}{llll}\text { C } & -2.45150800 & 3.39826600 & 1.12937800\end{array}$

$\begin{array}{llll}\mathrm{H} & -1.54867700 & 1.83144700 & 2.28635200\end{array}$

$\begin{array}{llll}\text { C } & -2.81212600 & 3.85270400 & -0.14159100\end{array}$

$\begin{array}{llll}\mathrm{H} & -2.80612900 & 3.39762700 & -2.25106800\end{array}$

H $\quad-2.67417700 \quad 4.00210400 \quad 2.00663000$

$\begin{array}{llll}\mathrm{H} & -3.31253800 & 4.81012900 & -0.26217500\end{array}$

$\begin{array}{llll}\mathrm{P} & 1.90509100 & 0.43083900 & -0.06295100\end{array}$

C $\quad 2.17304200 \quad 2.28602200 \quad-0.66091300$

$\begin{array}{llll}\text { C } & 2.78319100 & 0.23989200 & 1.66744900\end{array}$

C $\quad 2.85165400-0.69684200-1.36643700$

C $\quad 4.15363400 \quad-0.11615200-1.96111400$

$\mathrm{H} \quad 4.58029100-0.86527600 \quad-2.64166800$

$\mathrm{H} \quad 4.00616200 \quad 0.79421800 \quad-2.54421100$

$\mathrm{H} \quad 4.90589300 \quad 0.08473300 \quad-1.19336400$

C $\quad 3.22533800-2.07395800 \quad-0.77420900$

$\mathrm{H} \quad 3.59940100 \quad-2.70018800 \quad-1.59585400$

H $\quad 4.02711100-2.01400300-0.03492300$

H $\quad 2.37211300 \quad-2.59130000-0.33749400$

C $\quad 1.84869900-0.97087300-2.51283500$

H $\quad 2.33085800 \quad-1.61195500-3.26354400$

$\begin{array}{llll}\mathrm{H} & 0.97184200 & -1.50260100 & -2.13118100\end{array}$

H $\quad 1.51233600 \quad-0.06435500-3.02020400$

C $\quad 4.32624300 \quad 0.25862800 \quad 1.65092100$

$\begin{array}{llll}\mathrm{H} & 4.68667400 & 0.16812500 & 2.68488200\end{array}$

$\begin{array}{llll}\mathrm{H} & 4.75316800 & -0.57590400 & 1.09136000\end{array}$

$\begin{array}{llll}\mathrm{H} & 4.73600400 & 1.18510500 & 1.24438800\end{array}$

C $\quad 2.30057300 \quad 1.36109500 \quad 2.61462900$

$\begin{array}{llll}\mathrm{H} & 2.74115800 & 2.33269500 & 2.37901100\end{array}$

$\begin{array}{llll}\mathrm{H} & 1.20996700 & 1.46019000 & 2.61575500\end{array}$

$\begin{array}{llll}\mathrm{H} & 2.61039200 & 1.10681400 & 3.63683400\end{array}$

$\begin{array}{llll}\text { C } & 2.30526000 & -1.08879600 & 2.29716000\end{array}$

$\mathrm{H} \quad 2.59615000-1.97131700 \quad 1.72884700$

$\mathrm{H} \quad 2.74600300 \quad-1.17708500 \quad 3.29972100$

H $\quad 1.21629000-1.11358300 \quad 2.40454500$

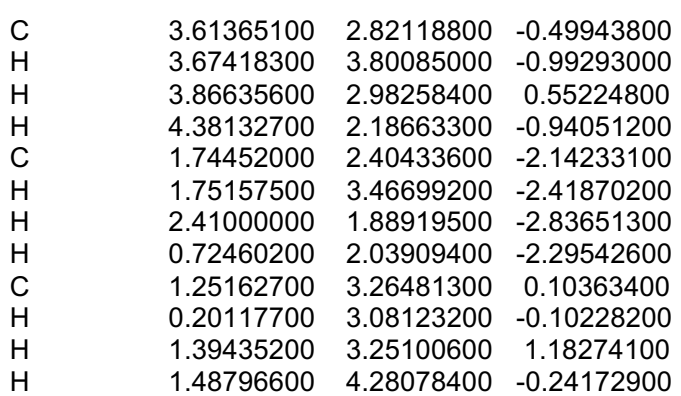

\section{Prod3}

Zero-point correction=

Thermal correction to Energy=

Thermal correction to Enthalpy=

0.545332 (Hartree/Particle)

Thermal correction to Gibbs Free Energy $=\quad 0.477513$

Sum of electronic and zero-point Energies $=\quad-1616.055777$

Sum of electronic and thermal Energies $=\quad-1616.019577$

Sum of electronic and thermal Enthalpies $=\quad-1616.018633$

Sum of electronic and thermal Free Energies $=-1616.123596$

Electronic energy (M06): -1616.148663

$\begin{array}{llll}\mathrm{Br} & -2.41894800 & 2.26706900 & 0.83690200\end{array}$

$\begin{array}{llll}\mathrm{P} & 1.46055200 & -0.97004100 & 0.07741700\end{array}$

$\begin{array}{llll}\text { C } & 0.54852800 & 2.16000400 & -0.26331600\end{array}$

$\begin{array}{llll}\text { C } & 0.93726900 & 3.01317600 & 0.77410500\end{array}$

C $\quad 0.76245900 \quad 2.54467800 \quad-1.59168500$

C $\quad 1.56110600 \quad 4.22953300 \quad 0.48181700$

$\begin{array}{llll}\mathrm{H} & 0.73829400 & 2.74872400 & 1.80802300\end{array}$

C $\quad \begin{array}{llll}1.38392300 & 3.76707900 & -1.87913000\end{array}$

$\mathrm{H} \quad 0.44483900 \quad 1.90247700 \quad-2.40942000$

C $\quad 1.79239300 \quad 4.60842000 \quad-0.84327400$

$\begin{array}{llll}\mathrm{H} & 1.85692300 & 4.88752200 & 1.29580500\end{array}$

$\begin{array}{llll}\mathrm{H} & 1.54524800 & 4.05574800 & -2.91540400\end{array}$

H $\quad 2.27590300 \quad 5.55599600 \quad-1.06580000$

C $\quad 3.24823500 \quad-0.33789900-0.44424500$

$\begin{array}{llll}\text { C } & 1.08716000 & -2.54618700 & -1.02290500\end{array}$

C $\quad 1.53918200 \quad-1.50344200 \quad 1.95430600$

$\mathrm{Na} \quad-3.95862900 \quad 0.53620200-0.46122100$

$\begin{array}{llll}\mathrm{Pd} & -0.52390200 & 0.50962700 & 0.02289300\end{array}$

$\begin{array}{llll}\text { C } & -2.92199700 & -1.67873300 & -0.91658200\end{array}$

C $\quad-0.01738300 \quad-3.40128100 \quad-0.36806900$

$\mathrm{H} \quad-0.87136800 \quad-2.79815800-0.06893900$

$\mathrm{H} \quad-0.36803900-4.13239600 \quad-1.10771200$

$\begin{array}{llll}\mathrm{H} & 0.34476200 & -3.96601200 & 0.49457600\end{array}$

C $\quad 0.51804100-2.03211100-2.36582700$

$\mathrm{H} \quad 0.27292800 \quad-2.89714200 \quad-2.99589600$

$\begin{array}{lll}0.40387000 & -1.46599100 & -2.21299300\end{array}$

$\mathrm{H} \quad 1.21910900-1.40883300 \quad-2.92337600$

$\begin{array}{llll}1.219778200 & -3.48742400 & -1.30156100\end{array}$

H $\quad \begin{array}{llll}2.89950100 & -4.34294600 & -1.87650100\end{array}$

H $\quad 3.06876900 \quad-3.02882700-1.89717200$

$\begin{array}{llll}H & 2.72084100 & -3.88727900 & -0.38533400\end{array}$

C $\quad 3.31634000 \quad-0.19838300 \quad-1.98235500$

$\begin{array}{llll}\mathrm{H} & 4.26019200 & 0.30225200 & -2.23482900\end{array}$

$\mathrm{H} \quad 3.30984400 \quad-1.15250800 \quad-2.51129400$

$\mathrm{H} \quad 2.50629300 \quad 0.42661000 \quad-2.36777300$

$\begin{array}{llll}\text { C } & 4.38798500 & -1.27195000 & 0.02671800\end{array}$

H $\quad 4.25198000 \quad-2.31746800 \quad-0.24682500$

H $\quad 5.32026300 \quad-0.93039300 \quad-0.44237500$

$\mathrm{H} \quad 4.53976700 \quad-1.21512400 \quad 1.10764000$

$\begin{array}{llll}\text { C } & 3.57160000 & 1.07243500 & 0.10661700\end{array}$

$\mathrm{H} \quad 3.02010500 \quad 1.84785700 \quad-0.41791300$

$\begin{array}{llll}\mathrm{H} & 3.38894600 & 1.19094200 & 1.17270900\end{array}$

H $\quad 4.64167800 \quad 1.25219300 \quad-0.06468900$

C $\quad 0.09311800 \quad-1.64329400 \quad 2.48599800$

$\mathrm{H} \quad-0.47827000 \quad-2.42424500 \quad 1.98596400$

$\mathrm{H} \quad 0.14282200 \quad-1.89534900 \quad 3.55388600$ 


$\begin{array}{lrrr}\mathrm{H} & -0.46498200 & -0.70664600 & 2.38622200 \\ \mathrm{C} & 2.19579500 & -0.38083000 & 2.78843800 \\ \mathrm{H} & 3.27093600 & -0.29504100 & 2.61635500 \\ \mathrm{H} & 1.73072600 & 0.59341300 & 2.60939400 \\ \mathrm{H} & 2.05756700 & -0.61932200 & 3.85102100 \\ \mathrm{C} & 2.29859900 & -2.81810600 & 2.23376600 \\ \mathrm{H} & 1.83161900 & -3.68356300 & 1.76017300 \\ \mathrm{H} & 3.34379800 & -2.78200000 & 1.92249200 \\ \mathrm{H} & 2.28774200 & -2.99924200 & 3.31691800 \\ \mathrm{O} & -2.25070000 & -1.09920800 & 0.02297200 \\ \mathrm{C} & -3.22616200 & -1.16986000 & -2.16457500 \\ \mathrm{H} & -3.80038300 & -1.76339600 & -2.86551000 \\ \mathrm{H} & -2.62142600 & -0.35675800 & -2.55838000 \\ \mathrm{O} & -3.49312800 & -2.90030000 & -0.62413800 \\ \mathrm{C} & -3.65387000 & -3.23165900 & 0.75069200 \\ \mathrm{H} & -4.31147500 & -2.51544700 & 1.26338400 \\ \mathrm{H} & -4.12123300 & -4.21887900 & 0.76156600 \\ \mathrm{H} & -2.70104200 & -3.26758100 & 1.28525300\end{array}$

\section{Prod4}

Zero-point correction= 0.545630 (Hartree/Particle) Thermal correction to Energy=

Thermal correction to Enthalpy= 0.581664 0.582609 0.478440 Sum of electronic and zero-point Energies $=\quad-1616.060011$ Sum of electronic and thermal Energies $=\quad-1616.023976$ Sum of electronic and thermal Enthalpies $=\quad-1616.023032$ Sum of electronic and thermal Free Energies $=-1616.127200$ Electronic energy (M06): -1616.14857

$\begin{array}{llll}\mathrm{Na} & 3.70368200 & -1.75006800 & -1.09037400\end{array}$

$\begin{array}{llll}\mathrm{Pd} & 0.57036100 & -0.02153900 & -0.26738000\end{array}$

$\begin{array}{llll}\text { C } & 3.52603900 & 0.18694000 & 0.53519700\end{array}$

C $\quad 0.67382900 \quad 1.95656200-0.49145200$

$\begin{array}{lllr}\text { C } & 1.11607600 & 2.78909200 & 0.54237500\end{array}$

C $\quad 0.50787600 \quad 2.48216800 \quad-1.77712300$

$\begin{array}{llll}\text { C } & 1.34795800 & 4.14732200 & 0.29665000\end{array}$

$\mathrm{H} \quad \begin{array}{llll}\mathrm{H} & 1.29804400 & 2.38283500 & 1.53207500\end{array}$

C $\quad 0.74388200 \quad 3.84099400 \quad-2.01765200$

$\begin{array}{llll}\mathrm{H} & 0.19937800 & 1.84215200 & -2.59938900\end{array}$

$\begin{array}{llll}\text { C } & 1.15475800 & 4.67989800 & -0.98017200\end{array}$

$\begin{array}{llll}\mathrm{H} & 1.68699300 & 4.78774400 & 1.10819600\end{array}$

$\mathrm{H} \quad 0.60633600 \quad 4.23938400 \quad-3.02053900$

$\mathrm{H} \quad \begin{array}{llll}\mathrm{H} & 1.33456400 & 5.73539000 & -1.16706700\end{array}$

$\begin{array}{llll}\mathrm{P} & -1.82821100 & -0.12292700 & 0.16726400\end{array}$

C $\quad-2.03730700 \quad-1.55539900 \quad 1.50363400$

C $\quad-2.68664000 \quad 1.47342100 \quad 0.92577500$

C $\quad-2.79786000-0.61562500-1.44635500$

C $\quad-3.35591700 \quad-1.54076900 \quad 2.30737100$

$\mathrm{H} \quad-3.34181700 \quad-2.40476400 \quad 2.98468500$

$\mathrm{H} \quad-3.48648100-0.65410400 \quad 2.92956900$

$\mathrm{H} \quad-4.23580200 \quad-1.65148700 \quad 1.66730200$

C $\quad-1.94256700 \quad-2.95582300 \quad 0.85906200$

$\begin{array}{llll}\mathrm{H} & -1.03976100 & -3.08872200 & 0.26491700\end{array}$

$\begin{array}{llll}H & -1.90402000 & -3.69196200 & 1.67296400\end{array}$

H $\quad-2.81518000 \quad-3.20308200 \quad 0.25060100$

$\begin{array}{llll}\text { C } & -0.84501700 & -1.43906100 & 2.48459900\end{array}$

$\begin{array}{llll}\mathrm{H} & -0.93910600 & -2.22943700 & 3.24150400\end{array}$

$\begin{array}{llll}H & 0.10874500 & -1.58317400 & 1.96926200\end{array}$

$\begin{array}{llll}\mathrm{H} & -0.80707600 & -0.48241900 & 3.00887800\end{array}$

C $\quad-2.95189900 \quad 0.62379700 \quad-2.35540100$

$\mathrm{H} \quad-3.71651700 \quad 1.31674200 \quad-1.99640000$

$\mathrm{H} \quad-2.01253500 \quad 1.17074000 \quad-2.47591200$

$\mathrm{H} \quad \begin{array}{llll}\mathrm{H} & -3.26757200 & 0.28374000 & -3.35018800\end{array}$

C $\quad-1.94482500-1.63371300 \quad-2.23528600$

$\mathrm{H} \quad-1.78651800 \quad-2.57287600-1.70671300$

$\mathrm{H} \quad-2.46448500-1.86215500-3.17574100$

$\mathrm{H} \quad-0.95848800-1.22964700 \quad-2.47904800$

C $\quad-4.20310700 \quad-1.21259100 \quad-1.21606600$

H $\quad-4.65642500-1.41155800-2.19656800$

$\begin{array}{lrrr}\mathrm{H} & -4.17813200 & -2.16271600 & -0.67976900 \\ \mathrm{H} & -4.87283500 & -0.53597600 & -0.68191700 \\ \mathrm{C} & -4.22692700 & 1.37329900 & 1.03065100 \\ \mathrm{H} & -4.58157900 & 2.22008300 & 1.63335300 \\ \mathrm{H} & -4.70255700 & 1.46584400 & 0.05037100 \\ \mathrm{H} & -4.59212700 & 0.46360200 & 1.50309600 \\ \mathrm{C} & -2.08712700 & 1.72549800 & 2.32836800 \\ \mathrm{H} & -2.43687500 & 2.70383500 & 2.68307800 \\ \mathrm{H} & -2.39091000 & 0.98721900 & 3.07175400 \\ \mathrm{H} & -0.99427800 & 1.76019500 & 2.29594600 \\ \mathrm{C} & -2.40019400 & 2.76187200 & 0.11628100 \\ \mathrm{H} & -1.35667000 & 3.05731900 & 0.16652000 \\ \mathrm{H} & -2.68910600 & 2.69947500 & -0.93106000 \\ \mathrm{H} & -2.99599000 & 3.56606700 & 0.56930900 \\ \mathrm{C} & 4.89296600 & 0.07788900 & 0.39842000 \\ \mathrm{H} & 5.56927500 & 0.09721100 & 1.24178100 \\ \mathrm{H} & 5.31840300 & 0.30933000 & -0.57439100 \\ \mathrm{O} & 2.71650800 & 0.17138400 & -0.48062300 \\ \mathrm{O} & 2.88689000 & 0.21026200 & 1.74501400 \\ \mathrm{C} & 3.67646800 & 0.26186600 & 2.92659000 \\ \mathrm{H} & 2.97204100 & 0.33835900 & 3.75715300 \\ \mathrm{H} & 4.27734900 & -0.64857800 & 3.04220800 \\ \mathrm{H} & 4.34049900 & 1.13454900 & 2.92162500\end{array}$

n3_2

Zero-point correction=

Thermal correction to Energy=

0.544122 (Hartree/Particle)

Thermal correction to Enthalpy=

Thermal correction to Gibbs Free Energy $=\quad 0.483611$

Sum of electronic and zero-point Energies $=\quad-1440.487970$

Sum of electronic and thermal Energies $=\quad-1440.456251$

Sum of electronic and thermal Enthalpies $=\quad-1440.455306$

Sum of electronic and thermal Free Energies $=-1440.548482$ Electronic energy (M06): -1440.608927

$\begin{array}{lrrr}\text { Pd } & -0.84498800 & -0.43993700 & -0.34282000 \\ \mathrm{P} & 1.42828500 & 0.06431700 & 0.08735300 \\ \mathrm{C} & -1.71922200 & 1.38429500 & -0.05744100 \\ \mathrm{C} & -2.45818100 & 1.64856100 & 1.10697800 \\ \mathrm{C} & -1.86816000 & 2.26096800 & -1.14313400 \\ \mathrm{C} & -3.30495300 & 2.75945800 & 1.18878400 \\ \mathrm{H} & -2.37880800 & 0.98514500 & 1.96617900 \\ \mathrm{C} & -2.71388900 & 3.37432800 & -1.06445900 \\ \mathrm{H} & -1.33234300 & 2.07569200 & -2.07213500 \\ \mathrm{C} & -3.43256000 & 3.63001100 & 0.10407800 \\ \mathrm{H} & -3.86709500 & 2.94217700 & 2.10263400 \\ \mathrm{H} & -2.81195100 & 4.03799900 & -1.92134300 \\ \mathrm{H} & -4.08877400 & 4.49443400 & 0.16786000 \\ \mathrm{O} & -2.82949400 & -1.37534200 & -0.67890700 \\ \mathrm{C} & -2.19587600 & -2.46612100 & -0.53011600 \\ \mathrm{C} & 2.37341400 & -0.28584300 & -1.59035800 \\ \mathrm{C} & 1.88469400 & 1.88624200 & 0.64924900 \\ \mathrm{C} & 2.10707600 & -1.15107800 & 1.46404900 \\ \mathrm{C} & -0.90845200 & -2.63360100 & -1.11270700 \\ \mathrm{H} & -0.34485500 & -3.52138500 & -0.84369200 \\ \mathrm{H} & -0.84005800 & -2.33065300 & -2.15500000 \\ \mathrm{O} & -2.64795100 & -3.41740300 & 0.31226200 \\ \mathrm{C} & -3.82385300 & -3.08608900 & 1.06678600 \\ \mathrm{H} & -4.67542700 & -2.91222200 & 0.40359400 \\ \mathrm{H} & -4.01099800 & -3.95133800 & 1.70437100 \\ \mathrm{H} & -3.65860400 & -2.19180400 & 1.67439100 \\ \mathrm{C} & 0.93443500 & 2.36845000 & 1.76946500 \\ \mathrm{H} & 1.00430300 & 1.78045600 & 2.68364000 \\ \mathrm{H} & 1.21564700 & 3.39892700 & 2.02511500 \\ \mathrm{H} & -0.10390700 & 2.37667500 & 1.44403300 \\ \mathrm{C} & 1.66677000 & 2.85368600 & -0.53602800 \\ \mathrm{H} & 1.77318600 & 3.87978700 & -0.16089200 \\ \mathrm{H} & 2.40025500 & 2.72945500 & -1.33496200 \\ \mathrm{H} & 0.66149600 & 2.76421900 & -0.95249900 \\ \mathrm{C} & 3.33167600 & 2.06033200 & 1.16348000\end{array}$




$\begin{array}{lrrr}\mathrm{H} & 3.50612800 & 3.13219500 & 1.32554100 \\ \mathrm{H} & 3.49169400 & 1.56684900 & 2.12506100 \\ \mathrm{H} & 4.09414100 & 1.71018700 & 0.46627700 \\ \mathrm{C} & 2.44498900 & -1.80514000 & -1.85560400 \\ \mathrm{H} & 1.46456700 & -2.27562000 & -1.77830800 \\ \mathrm{H} & 2.81189500 & -1.95683000 & -2.87913300 \\ \mathrm{H} & 3.13835900 & -2.32205100 & -1.18815100 \\ \mathrm{C} & 1.52583500 & 0.31583900 & -2.73785900 \\ \mathrm{H} & 1.43385700 & 1.40135100 & -2.68512300 \\ \mathrm{H} & 2.00716400 & 0.07155400 & -3.69430400 \\ \mathrm{H} & 0.51637800 & -0.10687600 & -2.74806300 \\ \mathrm{C} & 3.80998400 & 0.27031700 & -1.68500400 \\ \mathrm{H} & 4.23395800 & -0.03829700 & -2.64984600 \\ \mathrm{H} & 3.84974600 & 1.36064400 & -1.65528000 \\ \mathrm{H} & 4.46839600 & -0.11994500 & -0.90469800 \\ \mathrm{C} & 3.64332600 & -1.31360000 & 1.47426300 \\ \mathrm{H} & 3.91798000 & -1.95281200 & 2.32409900 \\ \mathrm{H} & 4.01662500 & -1.80586100 & 0.57314700 \\ \mathrm{H} & 4.17685700 & -0.36957700 & 1.59297700 \\ \mathrm{C} & 1.65536400 & -0.65791700 & 2.85704900 \\ \mathrm{H} & 1.85787800 & -1.45369500 & 3.58528800 \\ \mathrm{H} & 2.19648700 & 0.22850300 & 3.19345800 \\ \mathrm{H} & 0.58111400 & -0.44894000 & 2.88787700 \\ \mathrm{C} & 1.47788300 & -2.55134800 & 1.29389300 \\ \mathrm{H} & 1.72926700 & -3.02665200 & 0.34634700 \\ \mathrm{H} & 1.85919200 & -3.19390200 & 2.09886300 \\ \mathrm{H} & 0.38916500 & -2.51395300 & 1.37307300\end{array}$

n3_1

Zero-point correction= 0.544015 (Hartree/Particle)

Thermal correction to Energy=

Thermal correction to Enthalpy=

0.575883

Thermal correction to Gibbs Free Energy=
Sum of electronic and zero-point Energies= 0.576828

0.482554

Sum of electronic and thermal Energies=

$-1440.508055$

$-1440.476187$

Sum of electronic and thermal Enthalpies $=\quad-1440.475243$

Sum of electronic and thermal Free Energies $=-1440.569516$

Electronic energy (M06): -1440.626955

$\begin{array}{llll}\mathrm{Pd} & -0.94949400 & 0.35588900 & -0.41833400\end{array}$

$\begin{array}{llll}\mathrm{P} & 1.44788200 & -0.14286800 & 0.02780000\end{array}$

$\begin{array}{llll}\text { C } & -1.91285500 & -1.37225400 & -0.10246700\end{array}$

$\begin{array}{llll}\text { C } & -2.27498200 & -1.76781100 & 1.19410900\end{array}$

$\begin{array}{llll}\text { C } & -2.27689300 & -2.19775700 & -1.17807700\end{array}$

$\begin{array}{llll}\text { C } & -2.94933900 & -2.97507700 & 1.41213300\end{array}$

H $\quad-2.04025200 \quad-1.13488900 \quad 2.04589400$

C $\quad-2.95059500 \quad-3.40445600 \quad-0.95761600$

$\mathrm{H} \quad-2.03441800-1.90626500 \quad-2.19694900$

C $\quad-3.28613000 \quad-3.80106000 \quad 0.33847200$

$\mathrm{H} \quad-3.21646400 \quad-3.26368800 \quad 2.42670200$

H $\quad-3.21673800 \quad-4.03183200 \quad-1.80586200$

$\mathrm{H} \quad-3.81187400 \quad-4.73711600 \quad 0.50842000$

$\begin{array}{lllll}0 & -1.14368400 & 2.87276500 & -0.79600400\end{array}$

C $\quad-2.28263200 \quad 2.49653800 \quad-0.45169600$

C $\quad 2.17421100-1.80768400 \quad-0.67643000$

C $\quad 2.41116400 \quad 1.34083300 \quad-0.80678900$

$\begin{array}{llll}\text { C } & 1.76801700 & -0.08486700 & 1.94531700\end{array}$

$\begin{array}{lllll}\text { C } & -2.82336900 & 1.21578300 & -0.88656900\end{array}$

$\begin{array}{lllll}\mathrm{H} & -3.73863400 & 0.88611900 & -0.40268300\end{array}$

$\mathrm{H} \quad-2.83264300 \quad 1.09337300 \quad-1.97125300$

$\begin{array}{lllll}\mathrm{O} & -3.01779500 & 3.19053100 & 0.44003000\end{array}$

C $\quad-2.39492700 \quad 4.35410500 \quad 1.00314100$

$\mathrm{H} \quad-2.13070300 \quad 5.07078900 \quad 0.22090800$

$\mathrm{H} \quad-3.13673100 \quad 4.78303200 \quad 1.67795100$

$\mathrm{H} \quad \begin{array}{llll}\mathrm{H} & -1.49148600 & 4.08102100 & 1.55639600\end{array}$

C $\quad 3.93367600 \quad 1.14506000 \quad-0.95943400$

$\mathrm{H} \quad 4.18826600 \quad 0.32976300 \quad-1.64065900$

$\begin{array}{llll}\mathrm{H} & 4.36304600 & 2.06209600 & -1.38519900\end{array}$

$\mathrm{H} \quad 4.43577300 \quad 0.96446300 \quad-0.00526600$

C $\quad \begin{array}{llll}\text { C } & 1.78989000 & 1.58588700 & -2.20533900\end{array}$

H $\quad 0.73120900 \quad 1.85001100 \quad-2.12968600$

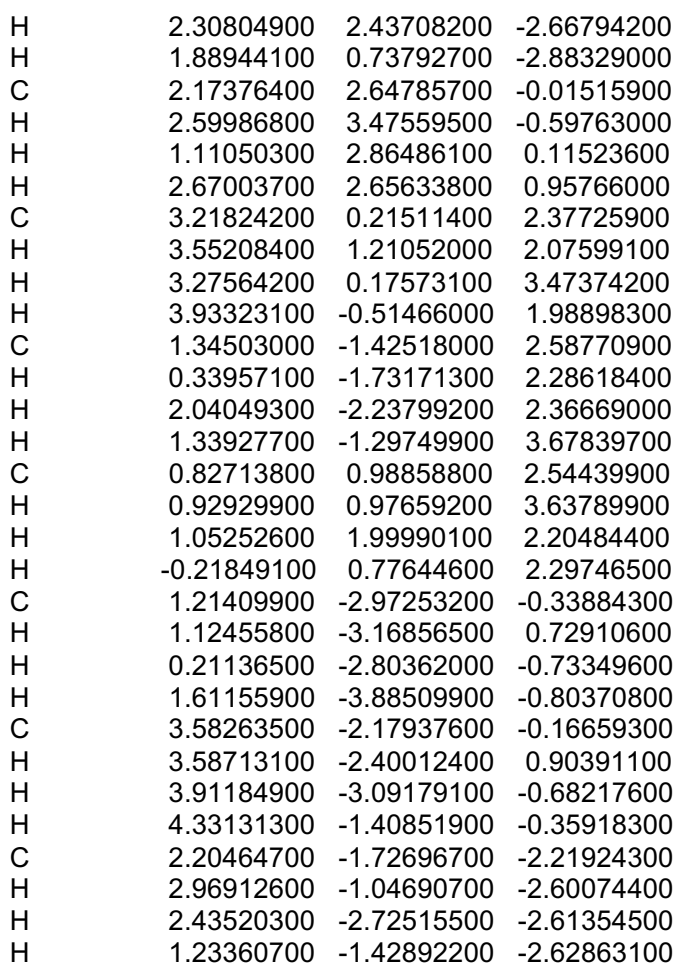

Nitronate reactivity

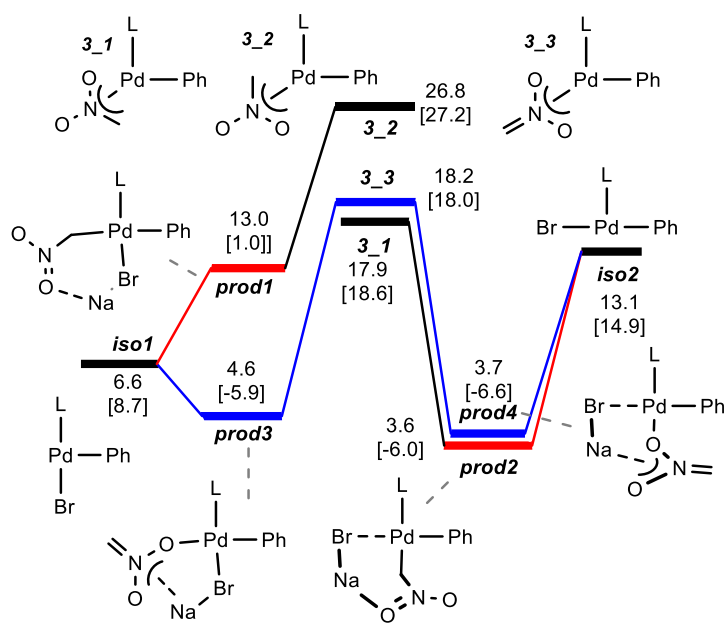

Nitronate

Zero-point correction=

Thermal correction to Energy=

0.038781 (Hartree/Particle)

Thermal correction to Enthalpy=

0.043896

0.044840

Thermal correction to Gibbs Free Energy=

Sum of electronic and zero-point Energies=

0.010292

Sum of electronic and thermal Energies=

$-406.706915$

$-406.701800$

Sum of electronic and thermal Enthalpies $=\quad-406.700856$

Sum of electronic and thermal Free Energies $=-406.735405$

Electronic energy (M06): -406.6865647

$\begin{array}{crrr}\mathrm{Na} & 1.88098000 & -0.00000300 & -0.00006400 \\ \mathrm{O} & 0.03275000 & -1.12193800 & -0.00006000 \\ \mathrm{C} & -1.95681000 & -0.00004700 & 0.00012700 \\ \mathrm{H} & -2.46141300 & 0.95341500 & 0.00017300 \\ \mathrm{H} & -2.46133500 & -0.95354900 & 0.00013200 \\ \mathrm{O} & 0.03267100 & 1.12198800 & -0.00001000 \\ \mathrm{~N} & -0.65007700 & 0.00000700 & 0.00002800\end{array}$




\section{Prod1}

Zero-point correction=

Thermal correction to Energy=

Thermal correction to Enthalpy=

0.506756 (Hartree/Particle)

0.540387

0.541331

Thermal correction to Gibbs Free Energy= 0.444061

Sum of electronic and zero-point Energies = $\quad-1592.738769$

Sum of electronic and thermal Energies $=\quad-1592.705138$

Sum of electronic and thermal Enthalpies $=\quad-1592.704194$

Sum of electronic and thermal Free Energies $=-1592.801464$ Electronic energy (M06): -1592.818797

$\begin{array}{lrrr}\mathrm{Br} & -2.95090800 & -0.85906600 & -1.58264700 \\ \mathrm{P} & 1.76920900 & 0.26319800 & -0.08719600 \\ \mathrm{C} & -1.44811900 & 1.39495800 & 0.19936000\end{array}$

$\begin{array}{llll}\text { C } & -1.44811900 & 1.39495800 & 0.19936000\end{array}$

C $\quad-1.71488500 \quad 1.51889600 \quad 1.57858400$

$\begin{array}{llll}\text { C } & -2.10285300 & 2.28906600 & -0.66529600\end{array}$

$\begin{array}{llll}\text { C } & -2.60169600 & 2.49445800 & 2.07275100\end{array}$

$\mathrm{H} \quad-1.21453000 \quad 0.87118900 \quad 2.29703400$

C $\quad-2.96543100 \quad 3.27218100-0.17614800$

H $\quad-1.96517600 \quad 2.20028200-1.73867400$

$\begin{array}{llll}\text { C } & -3.22542200 & 3.37860900 & 1.19492800\end{array}$

H $\quad-2.77607500 \quad 2.56835000 \quad 3.14451700$

$\mathrm{H} \quad-3.45421300 \quad 3.94837600 \quad-0.87406500$

$\mathrm{H} \quad-3.90227500 \quad 4.14176100 \quad 1.56960700$

C $\quad 2.29688700 \quad 0.54831200 \quad 1.76768500$

C $\quad 2.22563400 \quad 1.87546200-1.10668300$

C $\quad 2.86532000-1.19093600-0.82755100$

Pd $\quad-0.57332900-0.38875700-0.34668400$

$\mathrm{H} \quad-1.41358800 \quad-2.88726800-0.73353600$

$\mathrm{H} \quad 0.34562500-3.29138300-0.49126100$

C $\quad-0.50386300-2.69924200-0.17377100$

O $\quad 0.14912300-3.40273900 \quad 1.92714200$

O $\quad-1.83002900 \quad-2.52309400 \quad 1.72369500$

$\mathrm{Na} \quad-3.16812100 \quad-0.86222000 \quad 1.15829500$

$\begin{array}{llll}\mathrm{N} & -0.72780000 & -2.90692700 & 1.21028100\end{array}$

C $\quad 3.62993500 \quad 2.44307100 \quad-0.79848800$

$\mathrm{H} \quad 4.43739800 \quad 1.72147900-0.91807400$

$\mathrm{H} \quad 3.82388800 \quad 3.26737400 \quad-1.49762500$

$\begin{array}{llll}H & 3.69060200 & 2.86149600 & 0.20897800\end{array}$

C $\quad 1.20695800 \quad 3.00501100-0.84680100$

$\mathrm{H} \quad 0.22372500 \quad 2.74994400 \quad-1.23436600$

$\begin{array}{llll}\mathrm{H} & 1.09339000 & 3.27233800 & 0.20281900\end{array}$

H $\quad 1.55697400 \quad 3.89995400-1.37874600$

C $\quad 2.12303800 \quad 1.57036000-2.61843800$

$\mathrm{H} \quad 2.16276700 \quad 2.52242800-3.16304000$

$\mathrm{H} \quad 2.94347600 \quad 0.95516300 \quad-2.99181000$

H $\quad 1.17448700 \quad 1.08592300-2.87297000$

C $\quad 2.93400400 \quad-2.37321400 \quad 0.16778000$

H $\quad 1.97070800 \quad-2.67777400 \quad 0.57206100$

$\mathrm{H} \quad 3.35542600 \quad-3.23670700-0.36340400$

$\mathrm{H} \quad 3.60086300-2.16780400 \quad 1.00815200$

C $\quad 2.20015300-1.69978100-2.13093000$

$\mathrm{H} \quad 2.70770700-2.62312000-2.43896400$

$\mathrm{H} \quad 1.14061400-1.92377200-1.99941800$

$\mathrm{H} \quad 2.29198700 \quad-0.99112900-2.95428400$

C $\quad 4.32564100-0.81512700-1.16641500$

$\mathrm{H} \quad 4.83557400-1.72732400-1.50369200$

$\mathrm{H} \quad 4.40336600 \quad-0.09127600-1.97948200$

$\mathrm{H} \quad 4.88224000-0.43583900-0.30615300$

C $\quad 1.58564800 \quad-0.51886000 \quad 2.62874200$

$\mathrm{H} \quad 1.88876200 \quad-1.53918800 \quad 2.39926400$

$\mathrm{H} \quad 1.82336200-0.33197500 \quad 3.68446700$

$\begin{array}{llll}\mathrm{H} & 0.49893300 & -0.47085400 & 2.51811500\end{array}$

$\begin{array}{llll}\text { C } & 1.80544900 & 1.92810400 & 2.26043600\end{array}$

$\begin{array}{llll}\mathrm{H} & 2.38087700 & 2.75689600 & 1.84157100\end{array}$

$\begin{array}{llll}\mathrm{H} & 0.74768900 & 2.09750900 & 2.05143100\end{array}$

$\begin{array}{llll}\mathrm{H} & 1.93813600 & 1.96513000 & 3.34959000\end{array}$

C $\quad 3.81549800 \quad 0.47351000 \quad 2.04014000$

H $\quad 4.23352000 \quad-0.51699200 \quad 1.85400700$

$\mathrm{H} \quad 4.38824300 \quad 1.20358000 \quad 1.46420400$
$\mathrm{H}$

$3.98213900 \quad 0.69595700 \quad 3.10256400$

Prod2

Zero-point correction=

0.505938 (Hartree/Particle)

Thermal correction to Energy $=0.540048$

Thermal correction to Enthalpy= 0.540992

Thermal correction to Gibbs Free Energy $=\quad 0.440001$

Sum of electronic and zero-point Energies $=\quad-1592.752393$

Sum of electronic and thermal Energies $=\quad-1592.718284$

Sum of electronic and thermal Enthalpies $=\quad-1592.717340$

Sum of electronic and thermal Free Energies $=-1592.818331$

Electronic energy (M06): -1592.829679

$\mathrm{Br} \quad 0.57660800 \quad-3.17269800-0.40400300$

$\mathrm{Pd} \quad 0.78848800 \quad-0.29442800-0.31059200$

$\mathrm{H} \quad 2.83707500-1.72094300 \quad-1.17600900$

$\mathrm{H} \quad 3.00628300-0.03393500-1.81899600$

C $\quad 2.80905700 \quad-0.65645300-0.95029600$

O $\quad 4.53181900 \quad 0.63111200 \quad-0.07184200$

O $\quad 4.00321100-1.18169700 \quad 0.99420100$

$\mathrm{Na} \quad 2.33683700 \quad-2.49179200 \quad 1.48975700$

N $\quad 3.84618300 \quad-0.37318000 \quad 0.02745800$

C $\quad 1.47633000 \quad 1.58822500 \quad-0.15894400$

$\begin{array}{llll}\text { C } & 1.86844000 & 2.09701200 & 1.08681500\end{array}$

C $\quad 1.63665400 \quad 2.39128900-1.29610200$

C $\quad 2.37643100 \quad 3.39681500 \quad 1.19713800$

H $\quad 1.78052400 \quad 1.48825300 \quad 1.98397400$

C $\quad 2.13993300 \quad 3.69115500-1.18355300$

$\mathrm{H} \quad 1.37052700 \quad 2.01072300 \quad-2.27935500$

C $\quad 2.50655800 \quad 4.20095500 \quad 0.06390700$

$\mathrm{H} \quad 2.67237200 \quad 3.77613700 \quad 2.17268400$

$\begin{array}{llll}\mathrm{H} & 2.25180300 & 4.30250700 & -2.07638000\end{array}$

H $\quad 2.90208500 \quad 5.20955800 \quad 0.14956700$

$\begin{array}{llll}\mathrm{P} & -1.74631100 & 0.27657100 & 0.06793500\end{array}$

C $\quad-2.20762000 \quad 2.05067100 \quad 0.78291300$

C $\quad-2.58673100 \quad-1.02506400 \quad 1.28122000$

C $\quad-2.57175500 \quad 0.10620900-1.68842300$

C $\quad-3.94868700 \quad-0.61975600 \quad 1.88558400$

H $\quad-4.29770200 \quad-1.44784100 \quad 2.51697700$

$\mathrm{H} \quad-3.90600400 \quad 0.26604400 \quad 2.52029800$

$\mathrm{H} \quad-4.71201100 \quad-0.46005600 \quad 1.11915000$

C $\quad-2.81164000 \quad-2.39121200 \quad 0.59648100$

H $\quad-3.12464200 \quad-3.10509600 \quad 1.37059000$

$\mathrm{H} \quad-3.61018400-2.36396800-0.14793300$

$\mathrm{H} \quad-1.90759700 \quad-2.78937000 \quad 0.13864000$

C $\quad-1.57695900 \quad-1.26708900 \quad 2.42873000$

$\mathrm{H} \quad-1.33416100 \quad-0.36328800 \quad 2.99071500$

$\mathrm{H} \quad-2.00448600 \quad-1.99320100 \quad 3.13345200$

$\mathrm{H} \quad-0.64946800 \quad-1.69036700 \quad 2.03159800$

C $\quad-4.10818900-0.03851500-1.69715200$

$\begin{array}{lll}-4.44569500 & -0.09705100 & -2.74091700\end{array}$

$\mathrm{H} \quad-4.45159400-0.94828500-1.20074700$

$\mathrm{H} \quad-4.61674200 \quad 0.81179400 \quad-1.23902700$

C $\quad-1.94614500-1.12126300-2.38945500$

$\mathrm{H} \quad-2.15213600-2.06424800-1.88439900$

$\mathrm{H} \quad-2.35794100-1.18906400 \quad-3.40559000$

$\mathrm{H} \quad-0.85877100-1.02511800 \quad-2.47153500$

C $\quad-2.20197400 \quad 1.33221300 \quad-2.55223300$

$\mathrm{H} \quad-2.74502300 \quad 2.23381700-2.26024600$

$\mathrm{H} \quad-1.12870900 \quad 1.54599100 \quad-2.53089900$

$\mathrm{H} \quad-2.47464500 \quad 1.11469000-3.59315500$

$\begin{array}{llll}\text { C } & -1.40001600 & 3.18196200 & 0.10233700\end{array}$

$\mathrm{H} \quad-0.34458600 \quad 3.13905300 \quad 0.35422400$

$\mathrm{H} \quad-1.49227800 \quad 3.20241200 \quad-0.98191200$

H $\quad-1.79227500 \quad 4.13636700 \quad 0.47939800$

C $\quad-1.80124100 \quad 2.10619600 \quad 2.27392500$

$\mathrm{H} \quad-1.89844300 \quad 3.14392900 \quad 2.61853300$

$\begin{array}{llll}\mathrm{H} & -2.42613200 & 1.49286900 & 2.92483800\end{array}$

$\mathrm{H} \quad-0.75550900 \quad 1.81587800 \quad 2.41404300$

$\begin{array}{llll}\text { C } & -3.69840700 & 2.43673800 & 0.63760600\end{array}$

$\mathrm{H} \quad-3.87213100 \quad 3.35511700 \quad 1.21448600$ 
$\begin{array}{llll}\mathrm{H} & -3.95312900 & 2.66551100 & -0.40128800\end{array}$

H $\quad-4.39867800 \quad 1.68688200 \quad 1.00212200$

Prod3

Zero-point correction=

$\begin{array}{lc}\text { Thermal correction to Energy= } & 0.539944 \\ \text { Thermal correction to Enthalpy= } & 0.540888 \\ \text { Thermal correction to Gibbs Free Energy= } & 0.441382 \\ \text { Sum of electronic and zero-point Energies }= & -1592.756825 \\ \text { Sum of electronic and thermal Energies }= & -1592.722924 \\ \text { Sum of electronic and thermal Enthalpies }= & -1592.721980 \\ \text { Sum of electronic and thermal Free Energies }= & -1592.821486\end{array}$

Electronic energy (M06): -1592.829409

$\begin{array}{lllll}\mathrm{Br} & -3.26993200 & 0.37531100 & -1.00084500\end{array}$

P $\quad \begin{array}{llll}1.69901700 & -0.08788100 & -0.08300100\end{array}$

$\begin{array}{llll}\text { C } & -0.85452900 & 1.94634300 & 0.30094200\end{array}$

C $\quad \begin{array}{llll}-0.94998000 & 2.25566400 & 1.66096100\end{array}$

$\begin{array}{llll}\text { C } & -0.96948300 & 2.95920200 & -0.65551700\end{array}$

C $\quad-1.13393900 \quad 3.58425000 \quad 2.06365400$

$\begin{array}{llll}\mathrm{H} & -0.88179000 & 1.47458400 & 2.41339100\end{array}$

C $\quad-1.14823200 \quad 4.28463700 \quad-0.24470100$

H $\quad-0.94707300 \quad 2.72348100 \quad-1.71456800$

$\begin{array}{lllr}\text { C } & -1.22487700 & 4.60245900 & 1.11358700\end{array}$

$\begin{array}{llll}\mathrm{H} & -1.20406400 & 3.81677800 & 3.12378800\end{array}$

$\begin{array}{llll}\mathrm{H} & -1.23977400 & 5.06721700 & -0.99434000\end{array}$

$\begin{array}{llll}\mathrm{H} & -1.36563800 & 5.63325100 & 1.42750200\end{array}$

$\begin{array}{llll}\text { C } & 2.27180300 & -0.96354400 & 1.56023200\end{array}$

C $\quad 2.77250700 \quad 1.53972800 \quad-0.30369700$

$\begin{array}{llll}C & 2.09219100 & -1.24153700 & -1.61582000\end{array}$

$\begin{array}{llll}\mathrm{Pd} & -0.75375400 & 0.02230400 & -0.18785100\end{array}$

$\begin{array}{llll}\mathrm{O} & -2.12281600 & -3.15932300 & 1.74573600\end{array}$

$\mathrm{Na} \quad-3.39327200 \quad-1.69229300 \quad 0.71321200$

$\begin{array}{llll}\mathrm{N} & -1.44248500 & -3.40201700 & 0.66594700\end{array}$

C $\quad 4.28346400 \quad 1.31110400 \quad-0.06538400$

$\begin{array}{llll}\mathrm{H} & 4.70311900 & 0.48756300 & -0.64230000\end{array}$

$\mathrm{H} \quad 4.81587800 \quad 2.22412200 \quad-0.36294700$

$\begin{array}{llll}\mathrm{H} & 4.51068100 & 1.14265900 & 0.99060100\end{array}$

$\begin{array}{llll}\text { C } & 2.33256500 & 2.67456000 & 0.65326900\end{array}$

$\begin{array}{llll}\mathrm{H} & 1.35628700 & 3.07386300 & 0.39151700\end{array}$

$\begin{array}{llll}H & 2.31403900 & 2.38910200 & 1.70307900\end{array}$

$\begin{array}{llll}\mathrm{H} & 3.06270500 & 3.48870600 & 0.55014000\end{array}$

C $\quad 2.56989200 \quad 2.09633800 \quad-1.73073700$

$\mathrm{H} \quad 3.03704100 \quad 3.08839700 \quad-1.77853900$

$\mathrm{H} \quad 3.03635000 \quad 1.48504900 \quad-2.50538200$

$\mathrm{H} \quad \begin{array}{llll}\mathrm{H} & 1.51041000 & 2.22323700 & -1.96890600\end{array}$

C $\quad 1.63641100 \quad-2.68316700 \quad-1.31117800$

$\mathrm{H} \quad 0.59044300 \quad-2.71432000-1.00058200$

$\mathrm{H} \quad 1.72778500 \quad-3.26697900 \quad-2.23716400$

$\mathrm{H} \quad 2.25620400 \quad-3.17818000 \quad-0.56011400$

C $\quad 1.22615200-0.76578600 \quad-2.80911500$

$\mathrm{H} \quad 1.41728700-1.43268700 \quad-3.66028600$

$\mathrm{H} \quad 0.15725000-0.82175800 \quad-2.57884300$

$\mathrm{H} \quad \begin{array}{llll}\mathrm{H} & 1.44814300 & 0.25117900 & -3.13241200\end{array}$

C $\quad 3.56928300-1.29846200 \quad-2.05942000$

$\mathrm{H} \quad 3.65003800-2.03534000-2.86945300$

$\mathrm{H} \quad 3.93830300 \quad-0.35046700 \quad-2.45607400$

$\mathrm{H} \quad 4.23914300 \quad-1.62418800 \quad-1.25928700$

C $\quad \begin{array}{llll}\text { C } & 1.19851900 & -1.99782300 & 1.95971700\end{array}$

H $\quad 1.11592100 \quad-2.82822300 \quad 1.25986500$

$\begin{array}{llll}\mathrm{H} & 1.47336200 & -2.42133200 & 2.93484200\end{array}$

H $\quad 0.20674700 \quad-1.54764300 \quad 2.06141200$

$\begin{array}{llll}\text { C } & 2.32830300 & 0.06607200 & 2.71084400\end{array}$

$\begin{array}{llll}\mathrm{H} & 3.16755800 & 0.75948700 & 2.62546300\end{array}$

$\begin{array}{llll}\mathrm{H} & 1.40317800 & 0.64396800 & 2.79619700\end{array}$

$\begin{array}{llll}\text { H } & 2.45915300 & -0.48349500 & 3.65176900\end{array}$

C $\quad 3.63815100 \quad-1.67814000 \quad 1.48195600$

H $\quad 3.63864400 \quad-2.50435800 \quad 0.76803700$

$\mathrm{H} \quad 4.46082500-1.00670500 \quad 1.22919300$

$\begin{array}{llll}\mathrm{H} & 3.85907000 & -2.10729500 & 2.46839900\end{array}$

$\begin{array}{llll}\text { C } & -0.83611200 & -4.54572400 & 0.47593800\end{array}$
$\begin{array}{lrrr}\mathrm{H} & -0.31911900 & -4.70891800 & -0.45580900 \\ \mathrm{H} & -0.91843400 & -5.28796900 & 1.25523800 \\ \mathrm{O} & -1.43140300 & -2.45869800 & -0.26796400\end{array}$

Prod4

Zero-point correction= 0.505666 (Hartree/Particle)

Thermal correction to Energy $=\quad 0.539626$

Thermal correction to Enthalpy= $\quad 0.540570$

Thermal correction to Gibbs Free Energy $=\quad 0.440795$

Sum of electronic and zero-point Energies $=\quad-1592.760758$

Sum of electronic and thermal Energies $=\quad-1592.726798$

Sum of electronic and thermal Enthalpies $=\quad-1592.725854$

Sum of electronic and thermal Free Energies $=-1592.825629$

Electronic energy (M06): -1592.830229

$\mathrm{Br} \quad 0.65271300 \quad-3.09360800 \quad-0.40522300$

$\begin{array}{llll}\mathrm{Pd} & 0.67049400 & -0.27206800 & -0.17302800\end{array}$

$\begin{array}{llll}\mathrm{O} & 4.19816300 & -0.86250100 & 1.26195300\end{array}$

$\mathrm{Na} \quad 3.12001400 \quad-2.63571600 \quad 0.51916200$

$\begin{array}{llll}\mathrm{N} & 3.84180300 & -0.12483700 & 0.25911700\end{array}$

C $\quad 1.22204800 \quad 1.64025900-0.16349500$

$\begin{array}{llll}\text { C } & 1.63118200 & 2.26260400 & 1.02170900\end{array}$

$\begin{array}{llll}\text { C } & 1.40762800 & 2.30024400 & -1.38483800\end{array}$

$\begin{array}{llll}\text { C } & 2.18635700 & 3.54837500 & 0.98815300\end{array}$

$\begin{array}{llll}\mathrm{H} & 1.52761100 & 1.75360600 & 1.97632400\end{array}$

C $\quad 1.96659400 \quad 3.58274900-1.41470900$

$\mathrm{H} \quad 1.12713600 \quad 1.81914400 \quad-2.31810100$

$\begin{array}{lllll}\text { C } & 2.34824100 & 4.21489800 & -0.22755600\end{array}$

$\begin{array}{llll}\mathrm{H} & 2.49378800 & 4.02368400 & 1.91696200\end{array}$

$\mathrm{H} \quad 2.10286400 \quad 4.08694000 \quad-2.36896400$

$\begin{array}{llll}H & 2.77629200 & 5.21357600 & -0.25236300\end{array}$

$\begin{array}{llll}\mathrm{P} & -1.72911900 & 0.20323100 & 0.07679100\end{array}$

C $\quad-2.28802000 \quad 1.99924800 \quad 0.67145900$

C $\quad-2.44310100 \quad-1.05552100 \quad 1.41260100$

C $\quad-2.57590600 \quad-0.13503500-1.64521600$

$\begin{array}{llll}\text { C } & -3.80829400 & -0.67951400 & 2.03044000\end{array}$

H $\quad-4.08033800 \quad-1.47455800 \quad 2.73684600$

$\begin{array}{llll}\mathrm{H} & -3.80473500 & 0.25434600 & 2.59296300\end{array}$

H $\quad-4.60456600 \quad-0.63356900 \quad 1.28205100$

$\begin{array}{llll}\text { C } & -2.62363300 & -2.47514200 & 0.83148000\end{array}$

H $\quad-2.86453900 \quad-3.14448500 \quad 1.66801700$

H $\quad-3.45428900 \quad-2.54006200 \quad 0.12537700$

$\begin{array}{llll}\mathrm{H} & -1.72042100 & -2.86025800 & 0.36271400\end{array}$

C $\quad-1.38547100 \quad-1.16068900 \quad 2.53764900$

H $\quad-1.18116800 \quad-0.20753700 \quad 3.02959400$

$\mathrm{H} \quad-1.75724000 \quad-1.85545400 \quad 3.30239600$

$\mathrm{H} \quad-0.44237400 \quad-1.55914700 \quad 2.15288300$

C $\quad-4.10164900-0.36977300 \quad-1.59204500$

$\mathrm{H} \quad-4.46118000-0.50998200-2.62020600$

$-4.37233300-1.26943700-1.03643700$

H $\quad-4.65220900 \quad 0.47161200 \quad-1.16929200$

C $\quad-1.91092400-1.36900500-2.29303300$

$\mathrm{H} \quad-2.04297100 \quad-2.28574900-1.72033700$

$\mathrm{H} \quad-2.36517200-1.52262500-3.28120600$

$\mathrm{H} \quad-0.83622300 \quad-1.22441900 \quad-2.43192600$

$\begin{array}{llll}\text { C } & -2.30447000 & 1.05402800 & -2.59334900\end{array}$

$\begin{array}{llll}\mathrm{H} & -2.90342600 & 1.93436500 & -2.35021900\end{array}$

$\mathrm{H} \quad-1.24791300 \quad 1.33926300 \quad-2.60650400$

$\mathrm{H} \quad-2.57681600 \quad 0.74993500 \quad-3.61210000$

$\begin{array}{lllll}\text { C } & -1.60311000 & 3.15001100 & -0.10764900\end{array}$

$\begin{array}{llll}\mathrm{H} & -0.55695300 & 3.26004000 & 0.15982900\end{array}$

H $\quad-1.67147600 \quad 3.05921500-1.18961800$

$\mathrm{H} \quad-2.11558000 \quad 4.07978600 \quad 0.17440100$

$\begin{array}{llll}\text { C } & -1.86390300 & 2.19693900 & 2.14573700\end{array}$

$\begin{array}{llll}\mathrm{H} & -2.04451500 & 3.24607300 & 2.41417000\end{array}$

$\begin{array}{llll}\mathrm{H} & -2.42530300 & 1.58515000 & 2.85260300\end{array}$

H $\quad-0.79700500 \quad 2.00682100 \quad 2.28706100$

$\begin{array}{llll}\text { C } & -3.81052200 & 2.24991700 & 0.53863000\end{array}$

$\begin{array}{llll}\mathrm{H} & -4.04765700 & 3.17431500 & 1.08175000\end{array}$

$\begin{array}{llll}\mathrm{H} & -4.10060900 & 2.41518500 & -0.50260700\end{array}$ 


$\begin{array}{lrrr}\mathrm{H} & -4.44018300 & 1.46313800 & 0.94884600 \\ \mathrm{O} & 2.85365800 & -0.61001500 & -0.51782500 \\ \mathrm{C} & 4.43205800 & 0.99120500 & -0.05224700 \\ \mathrm{H} & 4.06385300 & 1.55370000 & -0.89423800 \\ \mathrm{H} & 5.24676300 & 1.31241900 & 0.57959200\end{array}$

\section{n3_1}

Zero-point correction=

Thermal correction to Energy=

Thermal correction to Enthalpy=

0.503733 (Hartree/Particle) 0.533680

0.534624

Thermal correction to Gibbs Free Energy $=\quad 0.444485$

Sum of electronic and zero-point Energies $=\quad-1417.178072$

Sum of electronic and thermal Energies $=\quad-1417.148125$

Sum of electronic and thermal Enthalpies $=\quad-1417.147181$

Sum of electronic and thermal Free Energies $=-1417.237320$

Electronic energy (M06): -1417.278613

c

c

$\mathrm{H}$

C

$\mathrm{H}$

C

$\mathrm{H}$

C

$\mathrm{H}$

$\mathrm{H}$

C

$\mathrm{H}$

$\mathrm{H}$

$\mathrm{H}$

C

$\mathrm{H}$

$\mathrm{H}$

C
$1.33404500-0.30611900-0.04109600$

$\begin{array}{lll}-2.18807100 & -0.74544000 & 0.02602500\end{array}$

$\begin{array}{lll}-2.84895700 & -1.03027500 & -1.17916000\end{array}$

$\begin{array}{lll}-2.50027400 & -1.51066400 & 1.15934800\end{array}$

$\begin{array}{lll}-3.77067200 & -2.08067900 & -1.25509800\end{array}$

$\begin{array}{llll}-2.64697300 & -0.43801700 & -2.06785000\end{array}$

$\begin{array}{lll}-3.42377200 & -2.55983600 & 1.08027400\end{array}$

$\begin{array}{lll}-2.03073700 & -1.29332700 & 2.11525700\end{array}$

$\begin{array}{lll}-4.05859100 & -2.85271500 & -0.12777900\end{array}$

$\begin{array}{llll}-4.26689800 & -2.28987800 & -2.20036500\end{array}$

$\begin{array}{lll}-3.64770200 & -3.14451200 & 1.97002000\end{array}$

$-4.77573900-3.66713900-0.18826000$

$\begin{array}{lll}1.87698100 & -0.90109000 & 1.73145300\end{array}$

$2.51828700 \quad 1.14473800-0.59978200$

$1.54868700-1.78454100-1.29100000$

$\begin{array}{lll}-0.89924200 & 0.78439000 & 0.14032800\end{array}$

$\begin{array}{lll}1.28953900 & 0.09324200 & 2.76218100\end{array}$

$\begin{array}{lll}1.52482300 & -0.26770200 & 3.77242900\end{array}$

$\begin{array}{lll}1.69467300 & 1.10118100 & 2.67123800\end{array}$

$\begin{array}{llll}0.20009000 & 0.16063400 & 2.67212700\end{array}$

$\begin{array}{llll}1.23653700 & -2.27146500 & 2.04636600\end{array}$

$\begin{array}{llll}0.15961400 & -2.28206200 & 1.85715700\end{array}$

$\begin{array}{lll}1.69715600 & -3.09125800 & 1.49056400\end{array}$

$\begin{array}{lll}1.38730900 & -2.48337800 & 3.11316700\end{array}$

$\begin{array}{llll}3.39811900 & -1.02244400 & 1.95854900\end{array}$

$\begin{array}{llll}3.91378600 & -0.06139000 & 1.89977900\end{array}$

$\begin{array}{llll}3.57302200 & -1.41752800 & 2.96855000\end{array}$

$\begin{array}{llll}3.87583400 & -1.70826900 & 1.25487900\end{array}$

$\begin{array}{lll}2.67553400 & 2.17095700 & 0.54652500\end{array}$

$3.27286500 \quad 1.79414600 \quad 1.37942600$

$\begin{array}{llll}3.20087700 & 3.04770200 & 0.14639000\end{array}$

$\begin{array}{lll}1.71248600 & 2.52078400 & 0.92615300\end{array}$

$3.92801600 \quad 0.71483300-1.05584400$

$4.51413600 \quad 1.61774700-1.27346500$

$3.90969900 \quad 0.11875400-1.97124000$

$\begin{array}{llll}1.83181800 & 1.91194500 & -1.75770300\end{array}$

$0.87923000 \quad 2.34615100-1.44173800$

$\begin{array}{llll}2.48474600 & 2.74328500 & -2.05564100\end{array}$

$\begin{array}{llll}1.65743400 & 1.30205100 & -2.64394100\end{array}$

$2.87982200-2.55525600-1.16640100$

$2.91869300 \quad-3.31447200 \quad-1.95925100$

$3.75929200-1.91912100-1.28157500$

$2.96370900-3.08565300-0.21453000$

$0.38666200-2.78906600-1.11091000$

$0.37102000-3.26811800 \quad-0.13268200$

$-0.58442100-2.31978100-1.27093800$

$0.50667200-3.58305100-1.86025700$

$1.41825300-1.24825900-2.73441700$

$2.27921300-0.65699400-3.05343300$

$1.34632200-2.10591800-3.41553000$

$0.51133900-0.64909400-2.86578500$

$\begin{array}{lll}-0.56766300 & 3.21979700 & 0.35745800\end{array}$

$\begin{array}{lll}-2.53133200 & 2.06253500 & 0.39532200\end{array}$
$4.46921000 \quad 0.15229500-0.29086700$

$\begin{array}{llll}\mathrm{H} & -2.67693100 & 2.07010500 & 1.47843800 \\ \mathrm{H} & -3.46323000 & 2.01237500 & -0.16201100 \\ \mathrm{O} & -2.28008000 & 4.18520400 & -0.60407100 \\ \mathrm{~N} & -1.79339100 & 3.26430900 & 0.02862000\end{array}$

n3_2

Zero-point correction=

Thermal correction to Energy $=\quad \begin{gathered}0.503431 \text { (Hartree/Particle) } \\ \text { The }\end{gathered}$

Thermal correction to Enthalpy $=0.534295$

Thermal correction to Gibbs Free Energy = 0.444711

Sum of electronic and zero-point Energies $=\quad-1417.160045$

Sum of electronic and thermal Energies $=\quad-1417.130126$

Sum of electronic and thermal Enthalpies $=\quad-1417.129181$

Sum of electronic and thermal Free Energies= -1417.218766 Electronic energy (M06): -1417.264651

\begin{tabular}{|c|c|c|c|}
\hline $\mathrm{P}$ & 1.25973900 & -0.31955000 & -0.02765300 \\
\hline $\mathrm{C}$ & -2.07228800 & -0.75393000 & 0.02785300 \\
\hline $\mathrm{C}$ & -2.73392300 & -1.00171400 & -1.18351100 \\
\hline $\mathrm{C}$ & -2.50163300 & -1.42766700 & 1.17907700 \\
\hline $\mathrm{C}$ & -3.78742100 & -1.92125700 & -1.24498100 \\
\hline $\mathrm{H}$ & -2.43828600 & -0.47565800 & -2.08820500 \\
\hline $\mathrm{C}$ & -3.55397900 & -2.34843700 & 1.11650700 \\
\hline $\mathrm{H}$ & -2.02869800 & -1.23532400 & 2.13946800 \\
\hline $\mathrm{C}$ & -4.19570900 & -2.60296800 & -0.09672500 \\
\hline $\mathrm{H}$ & -4.29031100 & -2.09993700 & -2.19296900 \\
\hline $\mathrm{H}$ & -3.87284800 & -2.86302100 & 2.0205580 \\
\hline $\mathrm{H}$ & -5.01225800 & -3.31877600 & -0.1453290 \\
\hline C & 1.94182500 & -0.63703100 & 1.7685720 \\
\hline C & 2.44895200 & 0.94010400 & -0.9507720 \\
\hline $\mathrm{C}$ & 1.32025100 & -2.00757500 & -1.0196860 \\
\hline $\mathrm{Pd}$ & -0.81070800 & 0.82209800 & 0.1645050 \\
\hline $\mathrm{C}$ & -0.44850700 & 3.08852100 & 0.5578470 \\
\hline $\mathrm{H}$ & -0.47924300 & 2.92455700 & 1.6329390 \\
\hline $\mathrm{H}$ & 0.19829700 & 3.89205000 & 0.2186810 \\
\hline $\mathrm{O}$ & -2.50841500 & 2.19673600 & 0.2796830 \\
\hline C & 1.52920900 & 0.55390600 & 2.6650780 \\
\hline $\mathrm{H}$ & 1.87291400 & 0.35384900 & 3.68851700 \\
\hline $\mathrm{H}$ & 1.96530000 & 1.50324500 & 2.35207900 \\
\hline $\mathrm{H}$ & 0.44078800 & 0.66918200 & 2.69011400 \\
\hline $\mathrm{C}$ & 1.26400300 & -1.88584200 & 2.37497600 \\
\hline $\mathrm{H}$ & 0.17562600 & -1.86053100 & 2.27231000 \\
\hline $\mathrm{H}$ & 1.63499600 & -2.81739200 & 1.94231100 \\
\hline $\mathrm{H}$ & 1.49548900 & -1.91453900 & 3.44751600 \\
\hline $\mathrm{C}$ & 3.47042600 & -0.83114200 & 1.86405200 \\
\hline $\mathrm{H}$ & 4.03153100 & 0.06003300 & 1.57608900 \\
\hline $\mathrm{H}$ & 3.72800600 & -1.04784800 & 2.9093440 \\
\hline $\mathrm{H}$ & 3.82882700 & -1.66871500 & 1.262398 \\
\hline $\mathrm{C}$ & 2.84213400 & 2.10490200 & -0.01475900 \\
\hline $\mathrm{H}$ & 3.54192100 & 1.80610100 & 0.76 \\
\hline $\mathrm{H}$ & 3.34454800 & 2.87075700 & -0.61954500 \\
\hline $\mathrm{H}$ & 1.97192700 & 2.57019900 & 0.44662400 \\
\hline C & 3.76064700 & 0.34286600 & -1.50428100 \\
\hline $\mathrm{H}$ & 4.34872900 & 1.16084100 & -1.94080200 \\
\hline $\mathrm{H}$ & 4.37780400 & -0.11864800 & -0.7286280 \\
\hline $\mathrm{H}$ & 3.59696600 & -0.38709600 & -2.2988420 \\
\hline C & 1.65448700 & 1.56692700 & -2.12246400 \\
\hline $\mathrm{H}$ & 0.75791800 & 2.07982300 & -1.76318100 \\
\hline $\mathrm{H}$ & 2.29392300 & 2.30845800 & -2.6193770 \\
\hline $\mathrm{H}$ & 1.35124200 & 0.83900000 & -2.87541600 \\
\hline C & 2.66252400 & -2.76397600 & -0.8924600 \\
\hline $\mathrm{H}$ & 2.64712800 & -3.60595800 & -1.5970150 \\
\hline $\mathrm{H}$ & 3.53953900 & -2.16174300 & -1.1274750 \\
\hline $\mathrm{H}$ & 2.79602200 & -3.19197800 & 0.1049100 \\
\hline C & 0.20840300 & -2.98425400 & -0.5707790 \\
\hline $\mathrm{H}$ & 0.23778900 & -3.23181300 & 0.4886780 \\
\hline $\mathrm{H}$ & -0.78406000 & -2.60527500 & -0.8014830 \\
\hline $\mathrm{H}$ & 0.35147000 & -3.91978400 & -1.1281360 \\
\hline $\mathrm{C}$ & 1.03640200 & -1.71467000 & -2.5106860 \\
\hline $\mathrm{H}$ & 1.85535500 & -1.20082000 & -3.0177840 \\
\hline
\end{tabular}




$\begin{array}{rrrr}\mathrm{H} & 0.88976900 & -2.67230700 & -3.02618400 \\ \mathrm{H} & 0.11881100 & -1.13203500 & -2.63886200 \\ \mathrm{~N} & -1.74514100 & 3.22180400 & 0.03048800 \\ \mathrm{O} & -2.11024400 & 4.13009300 & -0.70150200\end{array}$

n3_3

Zero-point correction $=$

Thermal correction to Energy $=\quad 0.504001$ (Hartree/Particle $)$

Thermal correction to Enthalpy= $\quad 0.534627$

Thermal correction to Gibbs Free Energy $=\quad 0.446047$

Sum of electronic and zero-point Energies $=\quad-1417.184179$

Sum of electronic and thermal Energies $=\quad-1417.154498$

Sum of electronic and thermal Enthalpies $=\quad-1417.153553$

Sum of electronic and thermal Free Energies $=-1417.242133$

Electronic energy (M06): -1417.279595

$\begin{array}{llll}\mathrm{P} & 1.25998500 & -0.30557200 & -0.01239100\end{array}$

C $\quad-2.01937800 \quad-0.85556200 \quad-0.02340800$

C $\quad-2.65341800-1.22179400-1.21934600$

C $\quad-2.42615300 \quad-1.45761900 \quad 1.17331000$

C $\quad-3.66284300 \quad-2.19064200-1.21719300$

H $\quad-2.37095900 \quad-0.74989400 \quad-2.15710200$

C $\quad-3.43616300 \quad-2.42755300 \quad 1.17112000$

H $\quad-1.97887500-1.16280300 \quad 2.11855000$

C $\quad-4.05247600 \quad-2.80202300 \quad-0.02343000$

$\mathrm{H} \quad-4.14593500 \quad-2.46331000 \quad-2.15295100$

$\begin{array}{llll}\mathrm{H} & -3.74288400 & -2.88368500 & 2.10989700\end{array}$

$\begin{array}{llll}\mathrm{H} & -4.83565300 & -3.55563400 & -0.02378200\end{array}$

C $\quad 1.75774400 \quad-0.67788400 \quad 1.83147900$

C $\quad 2.44333500 \quad 1.09425100 \quad-0.70901500$

C $\quad \begin{array}{llll}1.53873300 & -1.91585400 & -1.07498000\end{array}$

$\begin{array}{llll}\mathrm{Pd} & -0.85519800 & 0.76101300 & -0.02887800\end{array}$

$\begin{array}{llll}\text { O } & -2.52077200 & 2.11292200 & -0.00850300\end{array}$

$\begin{array}{llll}\text { C } & 1.12734400 & 0.41815500 & 2.72376300\end{array}$

$\begin{array}{llll}\mathrm{H} & 1.35840300 & 0.19044600 & 3.77300700\end{array}$

$\begin{array}{llll}\mathrm{H} & 1.50391500 & 1.41871700 & 2.51186800\end{array}$

$\begin{array}{llll}\mathrm{H} & 0.03888100 & 0.44395600 & 2.61176200\end{array}$

$\begin{array}{llll}\text { C } & 1.14785900 & -2.02057900 & 2.29326700\end{array}$

$\begin{array}{llll}\mathrm{H} & 0.07773600 & -2.08919400 & 2.08449100\end{array}$

$\begin{array}{llll}H & 1.64720800 & -2.88535700 & 1.85006200\end{array}$

H $\quad \begin{array}{llll}\text { H } & 1.27921800 & -2.09761200 & 3.38051000\end{array}$

C $\quad 3.27679900 \quad-0.73771900 \quad 2.09914900$

$\begin{array}{llll}\mathrm{H} & 3.77874300 & 0.21360900 & 1.91571100\end{array}$

H $\quad 3.43151900 \quad-0.98456800 \quad 3.15799700$

$\mathrm{H} \quad 3.77937100 \quad-1.50926800 \quad 1.51083100$

C $\quad \begin{array}{llll}2.57893100 & 2.24375700 & 0.31819000\end{array}$

$\begin{array}{llll}\mathrm{H} & 3.17399000 & 1.96863700 & 1.19197600\end{array}$

H $\quad 3.10545200 \quad 3.07002900-0.17721200$

$\begin{array}{llll}\mathrm{H} & 1.61185300 & 2.62979200 & 0.64466300\end{array}$

C $\quad 3.86473300 \quad 0.62219500 \quad-1.08292900$

$\mathrm{H} \quad 4.44819000 \quad 1.50345400 \quad-1.38067300$

$\mathrm{H} \quad 4.39387100 \quad 0.15014100 \quad-0.25143400$

H $\quad 3.87277100 \quad-0.06487000 \quad-1.93220900$

C $\quad 1.78569700 \quad 1.71884200 \quad-1.96659700$

$\begin{array}{llll}\mathrm{H} & 0.83667800 & 2.20284500 & -1.72403500\end{array}$

$\begin{array}{llll}\mathrm{H} & 2.46381400 & 2.49207700 & -2.35230200\end{array}$

H $\quad \begin{array}{llll}\text { H } & 1.61836500 & 1.00302400 & -2.77135800\end{array}$

$\begin{array}{llll}\text { C } & 2.89710800 & -2.61089200 & -0.82672500\end{array}$

H $\quad 3.00174000 \quad-3.42776900-1.55304500$

H $\quad 3.75791600 \quad-1.95319900-0.95010900$

$\begin{array}{llll}\mathrm{H} & 2.95120800 & -3.06286800 & 0.16709900\end{array}$

$\begin{array}{llll}\text { C } & 0.42966600 & -2.96143700 & -0.81177200\end{array}$

$\begin{array}{llll}\mathrm{H} & 0.37163700 & -3.28976300 & 0.22488200\end{array}$

$\mathrm{H} \quad-0.55380800 \quad-2.59769800 \quad-1.10471800$

$\mathrm{H} \quad 0.65809000 \quad-3.84575400 \quad-1.42187200$

C $\quad 1.42645900-1.55223500-2.57259600$

$\mathrm{H} \quad 2.27487800 \quad-0.97090700 \quad-2.93874700$

$\mathrm{H} \quad 1.39867300 \quad-2.48421700-3.15138700$

$\mathrm{H} \quad 0.50478300 \quad-1.00279500 \quad-2.78918600$

N $\quad-1.78423300 \quad 3.20673300 \quad 0.01976700$

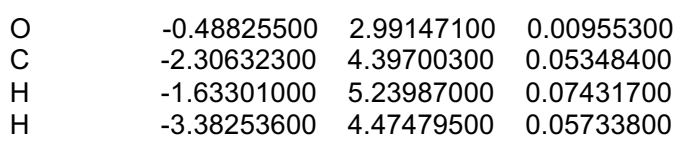

C-C REDUCTIVE ELIMINATIONS
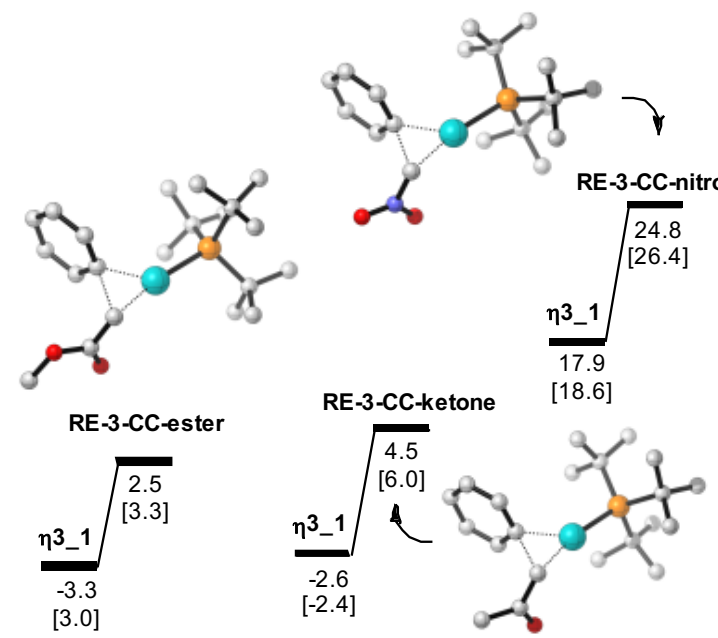

[3.0]

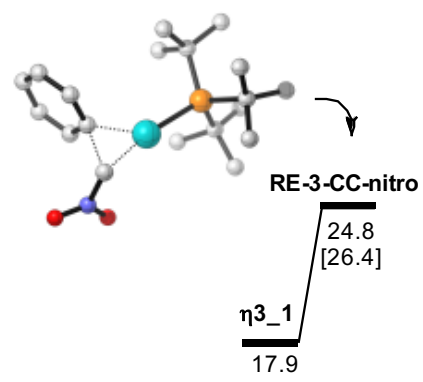

slow

fast

C-C reductive elimination

soft

enolate C center

\section{RE-3 CC ester}

Zero-point correction=

hard

Thermal correction to Energy=

Thermal correction to Enthalpy=

0.542934 (Hartree/Particle)

Thermal correction to Gibbs Free Energy $=\quad 0.480420$

Sum of electronic and zero-point Energies $=\quad-1440.485882$

Sum of electronic and thermal Energies $=\quad-1440.454327$

Sum of electronic and thermal Enthalpies $=\quad-1440.453383$

Sum of electronic and thermal Free Energies $=-1440.548396$ Electronic energy (M06): -1440.615616

$\begin{array}{lrrr}\text { C } & 2.10778100 & 0.78131500 & -0.29697700 \\ \mathrm{C} & 2.59785700 & 0.96198300 & 1.00744100 \\ \mathrm{C} & 2.27402700 & 1.81250300 & -1.23766000 \\ \mathrm{C} & 3.17331700 & 2.18096600 & 1.37893100 \\ \mathrm{C} & 2.84882200 & 3.02684900 & -0.85634300 \\ \mathrm{C} & 3.29702200 & 3.21910900 & 0.45345500 \\ \mathrm{H} & 3.53142600 & 2.31283500 & 2.39750800 \\ \mathrm{H} & 2.95292100 & 3.82243800 & -1.59068800 \\ \mathrm{P} & -1.86727800 & -0.07570300 & 0.05196100 \\ \mathrm{C} & -2.78015700 & 0.75890000 & -1.45182600 \\ \mathrm{C} & -2.71076000 & -1.79179800 & 0.41821100 \\ \mathrm{H} & 1.94864600 & 1.66980100 & -2.26420000 \\ \mathrm{H} & 3.75297400 & 4.16184000 & 0.74377800 \\ \mathrm{H} & 2.53376900 & 0.15076700 & 1.72621600 \\ \mathrm{Pd} & 0.46939800 & -0.44150000 & -0.48616500 \\ \mathrm{C} & 3.41176200 & -1.64822800 & -0.15020200 \\ \mathrm{C} & 2.44046500 & -1.09574300 & -1.13422000 \\ \mathrm{H} & 2.91669700 & -0.67818200 & -2.01702200 \\ \mathrm{H} & 1.76120600 & -1.92022200 & -1.43605500 \\ \mathrm{O} & 3.12272600 & -2.28314700 & 0.84818000 \\ \mathrm{C} & -2.06518900 & 1.06412300 & 1.61770600 \\ \mathrm{C} & 5.68773400 & -1.82818000 & 0.42925600 \\ \mathrm{H} & 5.63960300 & -2.91591500 & 0.53368900 \\ \mathrm{H} & 6.64591700 & -1.52654100 & 0.00395100 \\ \mathrm{H} & 5.54692700 & -1.37257400 & 1.41368500 \\ \mathrm{O} & 4.69306300 & -1.36018100 & -0.48992800 \\ \mathrm{C} & -1.85826000 & 1.86988900 & -2.01103100\end{array}$




$\begin{array}{lrrr}\mathrm{H} & -1.72667600 & 2.70966400 & -1.32901100 \\ \mathrm{H} & -0.86642700 & 1.47290200 & -2.24864200 \\ \mathrm{H} & -2.30348000 & 2.26354300 & -2.93503700 \\ \mathrm{C} & -4.16876200 & 1.35861000 & -1.15258000 \\ \mathrm{H} & -4.59750200 & 1.74997300 & -2.08548100 \\ \mathrm{H} & -4.87110900 & 0.62113600 & -0.75534000 \\ \mathrm{H} & -4.11903600 & 2.19441500 & -0.44980100 \\ \mathrm{C} & -2.92375900 & -0.27190100 & -2.59296500 \\ \mathrm{H} & -3.27050500 & 0.25198100 & -3.49326600 \\ \mathrm{H} & -1.96512800 & -0.74305300 & -2.83514900 \\ \mathrm{H} & -3.65465900 & -1.05376300 & -2.37588300 \\ \mathrm{C} & -2.15714800 & -2.81890800 & -0.60023600 \\ \mathrm{H} & -2.54657900 & -3.81371500 & -0.34518800 \\ \mathrm{H} & -2.44444200 & -2.61064200 & -1.63108100 \\ \mathrm{H} & -1.06306000 & -2.86615100 & -0.55552100 \\ \mathrm{C} & -4.25197700 & -1.81242700 & 0.36989300 \\ \mathrm{H} & -4.64503500 & -1.60516000 & -0.62876900 \\ \mathrm{H} & -4.60444600 & -2.81486900 & 0.64917800 \\ \mathrm{H} & -4.70510400 & -1.10278300 & 1.06681200 \\ \mathrm{C} & -2.25829500 & -2.30274900 & 1.80447100 \\ \mathrm{H} & -2.58787400 & -3.34431400 & 1.91388400 \\ \mathrm{H} & -1.16848900 & -2.29056400 & 1.90789200 \\ \mathrm{H} & -2.69688400 & -1.74001300 & 2.63130600 \\ \mathrm{C} & -3.45777500 & 1.06016300 & 2.27970300 \\ \mathrm{H} & -3.72084400 & 0.08457300 & 2.69652100 \\ \mathrm{H} & -3.45499000 & 1.77400800 & 3.11481500 \\ \mathrm{H} & -4.25486100 & 1.36405400 & 1.59621400 \\ \mathrm{C} & -1.70560200 & 2.51602400 & 1.23217900 \\ \mathrm{H} & -2.46059400 & 2.98883200 & 0.59997100 \\ \mathrm{H} & -1.63753100 & 3.11170300 & 2.15175800 \\ \mathrm{H} & -0.73429200 & 2.57368200 & 0.73103800 \\ \mathrm{C} & -1.00575100 & 0.63642500 & 2.66134100 \\ \mathrm{H} & -1.03432100 & 1.34827800 & 3.49753600 \\ \mathrm{H} & -1.17731700 & -0.35708800 & 3.07553200 \\ \mathrm{H} & -0.00048900 & 0.65875700 & 2.23173200\end{array}$

\section{RE-3 CC ketone}

Zero-point correction=

Thermal correction to Enthalpy= $\quad 0.568470$

Thermal correction to Gibbs Free Energy $=\quad 0.475417$

Sum of electronic and zero-point Energies $=\quad-1365.262100$

Sum of electronic and thermal Energies $=\quad-1365.231367$

Sum of electronic and thermal Enthalpies $=\quad-1365.230423$

Sum of electronic and thermal Free Energies $=-1365.323476$

Electronic energy (M06): -1365.392116

$\begin{array}{lrrr}\mathrm{C} & 2.36108300 & 0.49122200 & -0.27827900 \\ \mathrm{C} & 2.76638700 & 0.69441600 & 1.05228400 \\ \mathrm{C} & 2.72795800 & 1.43644200 & -1.25352000 \\ \mathrm{C} & 3.45893600 & 1.85663200 & 1.40696800 \\ \mathrm{C} & 3.41776800 & 2.59527100 & -0.88863600 \\ \mathrm{C} & 3.78350900 & 2.81312900 & 0.44239600 \\ \mathrm{H} & 3.75091000 & 2.00712300 & 2.44385700 \\ \mathrm{H} & 3.67540400 & 3.32636100 & -1.65170800 \\ \mathrm{P} & -1.67321500 & 0.02478500 & 0.02436100 \\ \mathrm{C} & -2.89984600 & -0.54836200 & -1.37554700 \\ \mathrm{C} & -2.13930200 & -0.89541300 & 1.67769700 \\ \mathrm{H} & 2.46383600 & 1.27362700 & -2.29473600 \\ \mathrm{H} & 4.32938400 & 3.71044200 & 0.72120300 \\ \mathrm{H} & 2.54678600 & -0.05578300 & 1.80550100 \\ \mathrm{Pd} & 0.61431100 & -0.55163900 & -0.54359500 \\ \mathrm{C} & 3.36537500 & -2.06539500 & 0.06306500 \\ \mathrm{C} & 2.53137500 & -1.45835000 & -1.02154600 \\ \mathrm{H} & 3.09381000 & -1.13364100 & -1.89552600 \\ \mathrm{H} & 1.77279500 & -2.20970800 & -1.33087200 \\ \mathrm{O} & 2.85867400 & -2.59396400 & 1.04315900 \\ \mathrm{C} & -1.88102300 & 1.94020500 & 0.29009400 \\ \mathrm{C} & 4.87482500 & -1.99873000 & -0.10775600 \\ \mathrm{H} & 5.18132700 & -2.54418700 & -1.00961400 \\ \mathrm{H} & 5.19342400 & -0.95732400 & -0.23556500\end{array}$

$\begin{array}{lll}5.36739000 & -2.43303700 & 0.76497200\end{array}$

$-2.41573300-1.92630300-1.89015800$

$-3.03895100-2.22420900-2.74431300$

$-1.37619400-1.87379700 \quad-2.23362900$

$-2.48357200 \quad-2.71836400-1.14459700$

$\begin{array}{lll}-2.78739800 & 0.40540200 & -2.58596400\end{array}$

$\begin{array}{lll}-1.74742200 & 0.54468800 & -2.89891000\end{array}$

$-3.32989100-0.04047600 \quad-3.43018400$

$\begin{array}{lll}-3.23261000 & 1.38547000 & -2.40148500\end{array}$

$\begin{array}{lll}-4.38449900 & -0.65110900 & -0.97098000\end{array}$

$\begin{array}{lll}-4.97725800 & -0.93323800 & -1.85196900\end{array}$

$\begin{array}{lll}-4.55926000 & -1.41618300 & -0.21053900\end{array}$

$\begin{array}{lll}-4.78497600 & 0.29503600 & -0.59787500\end{array}$

$\begin{array}{lll}-3.33442500 & 2.45267800 & 0.32588900\end{array}$

$\begin{array}{lll}-3.32674800 & 3.53044500 & 0.53869500\end{array}$

$\begin{array}{lll}-3.84793200 & 2.32050700 & -0.63001000\end{array}$

$\begin{array}{lll}-3.93434800 & 1.97150300 & 1.10220700\end{array}$

$\begin{array}{lll}-1.17839500 & 2.34711600 & 1.60424800\end{array}$

$\begin{array}{lll}-1.71265000 & 2.01259000 & 2.49647900\end{array}$

$\begin{array}{lll}-0.15025000 & 1.97437400 & 1.64467000\end{array}$

$\begin{array}{lll}-1.13400800 & 3.44312400 & 1.64777300\end{array}$

$\begin{array}{lll}-1.11347400 & 2.67212800 & -0.83719200\end{array}$

$\begin{array}{lll}-1.54368000 & 2.51569000 & -1.82656800\end{array}$

$\begin{array}{lll}-1.14510600 & 3.75126000 & -0.63449500\end{array}$

$\begin{array}{lll}-0.06467300 & 2.36388400 & -0.86818400\end{array}$

$\begin{array}{lll}-0.91923100 & -0.80731600 & 2.62806800\end{array}$

$\begin{array}{lll}-1.13295400 & -1.40289100 & 3.52591900\end{array}$

$\begin{array}{lll}-0.02160600 & -1.21843200 & 2.15402100\end{array}$

$\begin{array}{lll}-0.69568800 & 0.20823000 & 2.95541100\end{array}$

$\begin{array}{lll}-2.32557400 & -2.40232400 & 1.39156800\end{array}$

$\begin{array}{lll}-2.40269800 & -2.92883800 & 2.35161800\end{array}$

$\begin{array}{lll}-3.23522600 & -2.62469500 & 0.82948400\end{array}$

$\begin{array}{lll}-1.46705800 & -2.82308000 & 0.85776900\end{array}$

$\begin{array}{lll}-3.39394700 & -0.37815000 & 2.40966700\end{array}$

$\begin{array}{lll}-3.57083700 & -1.00226200 & 3.29634400\end{array}$

$\begin{array}{lll}-3.27980800 & 0.64986100 & 2.76252300\end{array}$

$\begin{array}{lll}-4.29535600 & -0.42784100 & 1.79281900\end{array}$

\section{RE-3 CC nitro}

Zero-point correction=

Thermal correction to Energy=

Thermal correction to Enthalpy=

0.502221 (Hartree/Particle)

Sum of electronic and zero-point Energies $=\quad-1417.152249$

Sum of electronic and thermal Energies $=\quad-1417.122478$

Sum of electronic and thermal Enthalpies $=\quad-1417.121534$

Sum of electronic and thermal Free Energies $=-1417.212990$

Electronic energy (M06): -1417.264626

$\begin{array}{lrrr}\text { C } & 2.41893500 & 0.46075100 & -0.28324500 \\ \mathrm{C} & 2.86012300 & 0.62856400 & 1.03767300 \\ \mathrm{C} & 2.74722500 & 1.42483300 & -1.25186300 \\ \mathrm{C} & 3.55277300 & 1.78887900 & 1.39731800 \\ \mathrm{C} & 3.43551400 & 2.58104700 & -0.87931700 \\ \mathrm{C} & 3.83675600 & 2.77026900 & 0.44593600 \\ \mathrm{H} & 3.87717900 & 1.91650100 & 2.42715300 \\ \mathrm{H} & 3.66767300 & 3.33029000 & -1.63240700 \\ \mathrm{P} & -1.64682800 & 0.04646000 & 0.02607200 \\ \mathrm{C} & -2.87099800 & -0.66393600 & -1.30920700 \\ \mathrm{C} & -2.08959500 & -0.72477700 & 1.75647000 \\ \mathrm{H} & 2.46102900 & 1.27690900 & -2.28938200 \\ \mathrm{H} & 4.38420800 & 3.66503900 & 0.72906400 \\ \mathrm{H} & 2.66860400 & -0.14197900 & 1.77815000 \\ \mathrm{Pd} & 0.64425200 & -0.55524100 & -0.51562100 \\ \mathrm{C} & 2.54590900 & -1.43626300 & -1.03239900 \\ \mathrm{H} & 3.23717800 & -1.15775400 & -1.81961100 \\ \mathrm{H} & 1.81769100 & -2.18451800 & -1.40515100 \\ \mathrm{O} & 2.66149300 & -2.57857500 & 0.98307000 \\ \mathrm{C} & -1.86591800 & 1.97653100 & 0.11927700 \\ \mathrm{C} & -2.38183800 & -2.08014400 & -1.70219400 \\ \mathrm{H} & -3.00919900 & -2.45360600 & -2.52275500\end{array}$




$\begin{array}{lrrr}\mathrm{H} & -1.34529800 & -2.05484400 & -2.05766800 \\ \mathrm{H} & -2.44118400 & -2.80503500 & -0.89045100 \\ \mathrm{C} & -2.77150500 & 0.17937500 & -2.59911400 \\ \mathrm{H} & -1.73402700 & 0.29403700 & -2.92978500 \\ \mathrm{H} & -3.31695500 & -0.34178600 & -3.39666900 \\ \mathrm{H} & -3.21988000 & 1.17029200 & -2.49894800 \\ \mathrm{C} & -4.35205300 & -0.74061100 & -0.88610800 \\ \mathrm{H} & -4.94792300 & -1.10433200 & -1.73430700 \\ \mathrm{H} & -4.51529500 & -1.43616300 & -0.05922000 \\ \mathrm{H} & -4.75700800 & 0.23208600 & -0.59579600 \\ \mathrm{C} & -3.32290600 & 2.48159400 & 0.11883800 \\ \mathrm{H} & -3.31869000 & 3.57533500 & 0.22018700 \\ \mathrm{H} & -3.84584400 & 2.25043200 & -0.81233500 \\ \mathrm{H} & -3.91150100 & 2.08093700 & 0.94796300 \\ \mathrm{C} & -1.16076500 & 2.50795600 & 1.38697800 \\ \mathrm{H} & -1.68395700 & 2.24306500 & 2.30840700 \\ \mathrm{H} & -0.12504300 & 2.16055700 & 1.45378800 \\ \mathrm{H} & -1.13730700 & 3.60417100 & 1.33371900 \\ \mathrm{C} & -1.11034000 & 2.60953300 & -1.07463200 \\ \mathrm{H} & -1.54598600 & 2.36552900 & -2.04375000 \\ \mathrm{H} & -1.14540500 & 3.70203000 & -0.96678000 \\ \mathrm{H} & -0.05987400 & 2.30489100 & -1.08530800 \\ \mathrm{C} & -0.86973300 & -0.53160900 & 2.69106800 \\ \mathrm{H} & -1.07151300 & -1.04786600 & 3.63912400 \\ \mathrm{H} & 0.03398000 & -0.96738200 & 2.25326000 \\ \mathrm{H} & -0.66234400 & 0.51229600 & 2.92586700 \\ \mathrm{C} & -2.25475300 & -2.25407500 & 1.60853600 \\ \mathrm{H} & -2.31847600 & -2.69322000 & 2.61242900 \\ \mathrm{H} & -3.16480200 & -2.54009700 & 1.07657800 \\ \mathrm{H} & -1.39372800 & -2.70929900 & 1.10827100 \\ \mathrm{C} & -3.34967600 & -0.16067100 & 2.44410800 \\ \mathrm{H} & -3.52446700 & -0.71571900 & 3.37584200 \\ \mathrm{H} & -3.24153700 & 0.89201700 & 2.71771400 \\ \mathrm{H} & -4.24957400 & -0.26285200 & 1.83160500 \\ \mathrm{O} & 4.52534200 & -2.19258000 & -0.08598500 \\ \mathrm{~N} & 3.30406600 & -2.12143200 & 0.03189700 \\ & & & \\ & & & \end{array}$

\section{C-O REDUCTIVE ELIMINATIONS}

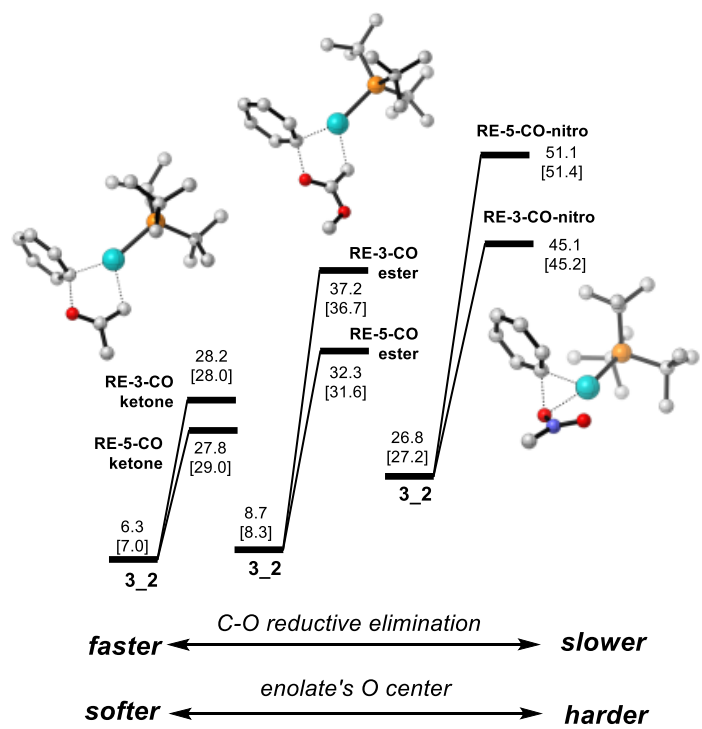

RE-3_CO_nitro

Zero-point correction $=$

Thermal correction to Energy=

Thermal correction to Enthalpy=

0.501492 (Hartree/Particle)

Thermal correction to Gibbs Free Energy $=\quad 0.443016$

Sum of electronic and zero-point Energies $=\quad-1417.133851$

Sum of electronic and thermal Energies=
Sum of electronic and thermal Enthalpies $=\quad-1417.103250$

Sum of electronic and thermal Free Energies $=-1417.192326$ Electronic energy (M06): -1417.233784

\begin{tabular}{|c|c|c|c|}
\hline ; & 2.31269000 & 0.47601400 & 0.23857500 \\
\hline C & 2.39529500 & 1.45561300 & 1.24145700 \\
\hline C & 2.83629800 & 0.71014500 & -1.04271300 \\
\hline C & 2.89820500 & 2.71444600 & 0.91488900 \\
\hline C & 3.33534800 & 1.98166200 & -1.34141000 \\
\hline C & 3.36431000 & 2.99055700 & -0.37634700 \\
\hline $\mathrm{H}$ & 2.93459900 & 3.48290600 & 1.68368200 \\
\hline $\mathrm{H}$ & 3.70755100 & 2.17528100 & -2.34482000 \\
\hline $\mathrm{P}$ & -1.54291700 & 0.02372100 & -0.02954500 \\
\hline C & -2.57999900 & -1.44202200 & -0.78775900 \\
\hline C & -1.64517000 & 1.55104100 & -1.22852400 \\
\hline $\mathrm{H}$ & 2.82333100 & -0.07599800 & -1.78986200 \\
\hline $\mathrm{H}$ & 3.76124200 & 3.97201800 & -0.61840800 \\
\hline $\mathrm{H}$ & 2.05983700 & 1.23425600 & 2.24881800 \\
\hline $\mathrm{Pd}$ & 0.63795400 & -0.80816700 & 0.29450000 \\
\hline C & -2.33724100 & 0.51968000 & 1.67974300 \\
\hline C & 4.59570700 & -2.16953800 & 0.18655400 \\
\hline $\mathrm{H}$ & 5.08181100 & -2.86177600 & -0.48488400 \\
\hline $\mathrm{O}$ & 2.72498900 & -1.12450400 & 0.95226400 \\
\hline $\mathrm{H}$ & 5.09318500 & -1.66479100 & 0.99874600 \\
\hline O & 2.57015600 & -2.43530700 & -0.87446800 \\
\hline C & -0.67639900 & 1.30824000 & -2.41064000 \\
\hline $\mathrm{H}$ & -0.64775700 & 2.21783000 & -3.02588500 \\
\hline $\mathrm{H}$ & 0.33873400 & 1.10793200 & -2.05912800 \\
\hline $\mathrm{H}$ & -0.98115300 & 0.48768000 & -3.06044000 \\
\hline C & -1.11046100 & 2.80319500 & -0.49794600 \\
\hline $\mathrm{H}$ & -1.78965300 & 3.16847200 & 0.27610100 \\
\hline $\mathrm{H}$ & -0.12648500 & 2.62714300 & -0.05267500 \\
\hline $\mathrm{H}$ & -0.99887000 & 3.61039700 & -1.23354300 \\
\hline C & -3.04694500 & 1.86444000 & -1.79026700 \\
\hline $\mathrm{H}$ & -2.98424300 & 2.77123900 & -2.40713700 \\
\hline $\mathrm{H}$ & -3.42580700 & 1.06669400 & -2.43457100 \\
\hline $\mathrm{H}$ & -3.78784000 & 2.05203700 & -1.00946100 \\
\hline C & -2.21203000 & -1.61765200 & -2.27760400 \\
\hline $\mathrm{H}$ & -2.64792600 & -2.56104800 & -2.63177500 \\
\hline $\mathrm{H}$ & -2.61081500 & -0.82283900 & -2.91182800 \\
\hline $\mathrm{H}$ & -1.12928100 & -1.67941500 & -2.42639200 \\
\hline C & -2.12594000 & -2.74895100 & -0.09094300 \\
\hline $\mathrm{H}$ & -2.63413400 & -3.59784300 & -0.56834400 \\
\hline $\mathrm{H}$ & -1.04539900 & -2.90316200 & -0.20222800 \\
\hline $\mathrm{H}$ & -2.36396700 & -2.78081600 & 0.97225400 \\
\hline C & -4.11316100 & -1.31502500 & -0.67801300 \\
\hline $\mathrm{H}$ & -4.49616800 & -0.41353500 & -1.16344800 \\
\hline $\mathrm{H}$ & -4.57742700 & -2.17644400 & -1.17740300 \\
\hline $\mathrm{H}$ & -4.46467100 & -1.31998800 & 0.35682000 \\
\hline C & -3.64475800 & 1.33321300 & 1.60143900 \\
\hline $\mathrm{H}$ & -4.02556200 & 1.49295000 & 2.61941500 \\
\hline $\mathrm{H}$ & -3.49432100 & 2.32128000 & 1.15982900 \\
\hline $\mathrm{H}$ & -4.42921900 & 0.82265700 & 1.03689200 \\
\hline C & -2.60052500 & -0.75087600 & 2.51897500 \\
\hline $\mathrm{H}$ & -2.87129900 & -0.44236600 & 3.53726300 \\
\hline $\mathrm{H}$ & -3.42601200 & -1.35641800 & 2.13856200 \\
\hline $\mathrm{H}$ & -1.70652700 & -1.37880000 & 2.59436000 \\
\hline C & -1.28585200 & 1.32600600 & 2.47967100 \\
\hline $\mathrm{H}$ & -0.36831400 & 0.74361500 & 2.60592700 \\
\hline $\mathrm{H}$ & -1.01937700 & 2.27685700 & 2.01838900 \\
\hline $\mathrm{H}$ & -1.69512000 & 1.54464400 & 3.47540600 \\
\hline $\mathrm{N}$ & 3.31822000 & -1.96905700 & 0.01696300 \\
\hline
\end{tabular}

RE-5_CO_nitro

Zero-point correction= 0.501238 (Hartree/Particle)

Thermal correction to Energy=

Thermal correction to Enthalpy=

0.530682

0.531626

Thermal correction to Gibbs Free Energy= $\quad 0.442322$

Sum of electronic and zero-point Energies $=\quad-1417.120277$

Sum of electronic and thermal Energies $=\quad-1417.090833$

Sum of electronic and thermal Enthalpies $=\quad-1417.089889$ 
Sum of electronic and thermal Free Energies $=-1417.179193$ Electronic energy (M06): -1417.223469

\begin{tabular}{|c|c|c|c|}
\hline $\mathrm{Pd}$ & -0.83622900 & -0.23456500 & -0.07399600 \\
\hline $\mathrm{P}$ & 1.60529800 & 0.10223600 & 0.00677400 \\
\hline C & -2.81340300 & 0.19307600 & -0.01930200 \\
\hline C & -3.37988600 & 0.74328200 & -1.19219800 \\
\hline C & -2.87982800 & 0.90364300 & 1.20463900 \\
\hline C & -3.89361800 & 2.03722000 & -1.15159400 \\
\hline $\mathrm{H}$ & -3.39243500 & 0.17267400 & -2.11597500 \\
\hline C & -3.39210000 & 2.20706800 & 1.20791000 \\
\hline $\mathrm{H}$ & -2.59045700 & 0.41712200 & 2.13150300 \\
\hline C & -3.89919900 & 2.77749800 & 0.03942700 \\
\hline $\mathrm{H}$ & -4.29770300 & 2.47300100 & -2.06248300 \\
\hline $\mathrm{H}$ & -3.42192300 & 2.75986500 & 2.14398300 \\
\hline $\mathrm{H}$ & -4.31535900 & 3.78043300 & 0.05596500 \\
\hline $\mathrm{O}$ & -3.55840800 & -1.50910100 & 0.32950700 \\
\hline C & 2.01283100 & 1.94399300 & 0.49603600 \\
\hline C & 2.44695800 & -1.08003100 & 1.30507800 \\
\hline C & 2.36859100 & -0.25348900 & -1.74719400 \\
\hline C & -1.58127500 & -2.15164800 & -0.74566300 \\
\hline $\mathrm{H}$ & -1.93637300 & -1.78786800 & -1.71270600 \\
\hline $\mathrm{H}$ & -0.96496600 & -3.04254800 & -0.82043300 \\
\hline $\mathrm{O}$ & -2.88191300 & -3.56265700 & 0.55981800 \\
\hline C & 1.05626400 & 2.35828300 & 1.64091300 \\
\hline $\mathrm{H}$ & 1.21573100 & 3.42226400 & 1.86204700 \\
\hline $\mathrm{H}$ & 0.00799500 & 997500 & 5107700 \\
\hline $\mathrm{H}$ & 1.22005600 & 1.80615100 & 2.56633900 \\
\hline C & 1.66568100 & 2.87616700 & -0.68638400 \\
\hline $\mathrm{H}$ & 2.36821200 & 2.79341400 & -1.51877600 \\
\hline $\mathrm{H}$ & 0.65201200 & 2.69857000 & -1.06111300 \\
\hline $\mathrm{H}$ & 1.70706000 & 3.91487200 & -0.33350600 \\
\hline C & 3.46598200 & 2.22555500 & 0.93017000 \\
\hline $\mathrm{H}$ & 3.57790100 & 558400 & 7300 \\
\hline $\mathrm{H}$ & 3.73703400 & 1.70376200 & 1.85159200 \\
\hline $\mathrm{H}$ & 4.19646800 & 1.95894300 & 0.16214400 \\
\hline C & 1.41059600 & 0.34354500 & -2.80735700 \\
\hline $\mathrm{H}$ & 0.39076400 & -0.03171400 & -2.67000200 \\
\hline $\mathrm{H}$ & 1.37232300 & 1.43288900 & -2.79373700 \\
\hline $\mathrm{H}$ & 1.75478200 & 0.04020000 & -3.80527300 \\
\hline C & 3.79265100 & 0.28581100 & -1.99313100 \\
\hline $\mathrm{H}$ & 4.12163200 & -0.01756400 & -2.99663200 \\
\hline $\mathrm{H}$ & 3.83973000 & 1.37710900 & -1.95954500 \\
\hline $\mathrm{H}$ & 4.52100400 & -0.10755000 & -1.27929300 \\
\hline C & 2.37568100 & -1.77701700 & -2.00613200 \\
\hline $\mathrm{H}$ & 2.64010100 & -1.94816200 & -3.05787000 \\
\hline $\mathrm{H}$ & 3.11085500 & 165100 & -1.40101000 \\
\hline $\mathrm{H}$ & 1.39085500 & -2.22337800 & -1.83700400 \\
\hline C & 3.97414700 & -1.24885600 & 1.17417800 \\
\hline $\mathrm{H}$ & 4.33329600 & -1.89247700 & 1.98886100 \\
\hline $\mathrm{H}$ & 4.26314600 & -1.73125400 & 0.23708100 \\
\hline $\mathrm{H}$ & 4.51256400 & -0.30038600 & 1.24728300 \\
\hline C & 2.13187700 & -0.58162000 & 2.73330200 \\
\hline $\mathrm{H}$ & 2.44986500 & -1.35250900 & 3.44726200 \\
\hline $\mathrm{H}$ & 2.66293100 & 0.33599300 & 2.99527500 \\
\hline $\mathrm{H}$ & 1.05893100 & -0.42131800 & 2.88250200 \\
\hline C & 1.76969400 & -2.46729000 & 1.19416900 \\
\hline $\mathrm{H}$ & 1.95974600 & -2.96961000 & 0.24527500 \\
\hline $\mathrm{H}$ & 2.16134500 & -3.11409300 & 1.99059900 \\
\hline $\mathrm{H}$ & 0.68591000 & -2.38658100 & 1.32080200 \\
\hline $\mathrm{N}$ & -2.69618400 & -2.45139700 & 0.07779800 \\
\hline
\end{tabular}

\section{RE-3 CO ester}

Zero-point correction $=$

Thermal correction to Energy=

Thermal correction to Enthalpy=

0.542328 (Hartree/Particle) 0.573667

0.574611

Thermal correction to Gibbs Free Energy $=\quad 0.481542$

Sum of electronic and zero-point Energies $=\quad-1440.443116$

Sum of electronic and thermal Energies $=\quad-1440.411777$

Sum of electronic and thermal Enthalpies $=\quad-1440.410833$

Sum of electronic and thermal Free Energies $=-1440.503902$
Electronic energy (M06): -1440.561477

$\begin{array}{lrrr}\mathrm{C} & -2.07742900 & 0.91850300 & -0.25139900 \\ \mathrm{C} & -2.06142900 & 2.01733800 & -1.12232900 \\ \mathrm{C} & -2.59259300 & 1.03820100 & 1.04582900 \\ \mathrm{C} & -2.45594500 & 3.26626500 & -0.64322100 \\ \mathrm{C} & -2.98556000 & 2.30033100 & 1.50265000 \\ \mathrm{C} & -2.91243200 & 3.41979500 & 0.67160300 \\ \mathrm{H} & -2.41946800 & 4.12381200 & -1.31129500 \\ \mathrm{H} & -3.35594600 & 2.39924000 & 2.52058500 \\ \mathrm{P} & 1.68186000 & -0.07280500 & 0.04030500 \\ \mathrm{C} & 2.52693200 & -1.78887400 & 0.41347200 \\ \mathrm{C} & 1.86711300 & 1.06797700 & 1.60593300 \\ \mathrm{H} & -2.66802400 & 0.16819000 & 1.68884000 \\ \mathrm{H} & -3.22668200 & 4.39455700 & 1.03326200\end{array}$

H $\quad-1.74377600 \quad 1.89121900-2.15176200$

Pd $\quad-0.55011200-0.51341800 \quad-0.54587000$

C $\quad-3.49005800 \quad-1.34876100 \quad-0.53279700$

C $\quad 2.61409000 \quad 0.76089700 \quad-1.45441400$

$\begin{array}{llll}\text { C } & -4.80981000 & -1.28623200 & -0.78766500\end{array}$

H $\quad-5.52849100-1.95426600 \quad-0.33268000$

$\begin{array}{llll}\mathrm{O} & -2.59997000 & -0.58452700 & -1.17423600\end{array}$

$\mathrm{H} \quad-5.16687200 \quad-0.56238700 \quad-1.50791800$

$\begin{array}{llll}\mathrm{O} & -2.88614000 & -2.18445000 & 0.37169800\end{array}$

$\begin{array}{llll}\text { C } & -3.72531300 & -3.06133800 & 1.10844500\end{array}$

$\mathrm{H} \quad-4.24757900 \quad-3.75999900 \quad 0.44229300$

H $\quad-3.07062600 \quad-3.61856900 \quad 1.78110700$

H $\quad-4.46738100 \quad-2.50130700 \quad 1.69225900$

$\begin{array}{llll}\text { C } & 0.81162000 & 0.63713300 & 2.65134400\end{array}$

$\mathrm{H} \quad 0.84506200 \quad 1.34696100 \quad 3.48912300$

$\begin{array}{llll}\mathrm{H} & -0.19506400 & 0.66374400 & 2.22752600\end{array}$

H $\quad 0.98658300 \quad-0.35717900 \quad 3.06239800$

$\begin{array}{llll}\text { C } & 1.50284500 & 2.51797400 & 1.21684200\end{array}$

$\begin{array}{llll}\mathrm{H} & 2.26013100 & 2.99230800 & 0.58847600\end{array}$

$\begin{array}{llll}\mathrm{H} & 0.53426300 & 2.57352700 & 0.71066600\end{array}$

$\begin{array}{llll}\mathrm{H} & 1.42822300 & 3.11413100 & 2.13557900\end{array}$

$\begin{array}{llll}\text { C } & 3.25905500 & 1.06741400 & 2.26993500\end{array}$

$\begin{array}{llll}\mathrm{H} & 3.25351000 & 1.78607500 & 3.10078400\end{array}$

$\begin{array}{llll}\mathrm{H} & 3.52000000 & 0.09408500 & 2.69359900\end{array}$

$\begin{array}{llll}\mathrm{H} & 4.05864600 & 1.36695000 & 1.58796900\end{array}$

$\begin{array}{llll}\text { C } & 2.06583000 & -2.30263900 & 1.79562600\end{array}$

$\begin{array}{llll}\mathrm{H} & 2.39386200 & -3.34478100 & 1.90490200\end{array}$

$\begin{array}{llll}\mathrm{H} & 2.49945400 & -1.74182100 & 2.62644900\end{array}$

$\begin{array}{llll}\mathrm{H} & 0.97536700 & -2.28788700 & 1.89077900\end{array}$

C $\quad 1.98640800 \quad-2.81716800-0.61146200$

H $\quad 2.37632300 \quad-3.81101900-0.35256700$

H $\quad 0.89129300 \quad-2.86820400 \quad-0.58049700$

H $\quad 2.28387600 \quad-2.60759700 \quad-1.63892000$

C $\quad 4.06873200 \quad-1.80501600 \quad 0.37937500$

$\mathrm{H} \quad 4.51392900 \quad-1.09709400 \quad 1.08302300$

$\begin{array}{llll}\mathrm{H} & 4.42030700 & -2.80772600 & 0.65886900\end{array}$

$\mathrm{H} \quad 4.47110800 \quad-1.59414300 \quad-0.61459800$

C $\quad 3.99540000 \quad 1.36690900-1.13429900$

$\begin{array}{llll}\mathrm{H} & 4.43900700 & 1.75029900 & -2.06345500\end{array}$

$\begin{array}{llll}\mathrm{H} & 3.93075700 & 2.20982500 & -0.44137700\end{array}$

$\mathrm{H} \quad 4.69339100 \quad 0.63570100 \quad-0.71834800$

$\begin{array}{llll}\text { C } & 2.77969400 & -0.27122700 & -2.59145500\end{array}$

$\mathrm{H} \quad 3.13370000 \quad 0.25433500 \quad-3.48789000$

H $\quad 3.51385700-1.04704000-2.36429200$

$\mathrm{H} \quad \begin{array}{llll}\mathrm{H} & 1.82722500 & -0.74886800 & -2.84425500\end{array}$

$\begin{array}{llll}\text { C } & 1.69737300 & 1.86590500 & -2.03121800\end{array}$

$\begin{array}{llll}\mathrm{H} & 0.72248600 & 1.45449500 & -2.30774600\end{array}$

H $\quad \begin{array}{llll}\text { H } & 1.52777700 & 2.69532800 & -1.34503200\end{array}$

$\begin{array}{llll}\mathrm{H} & 2.16874800 & 2.27590700 & -2.93485700\end{array}$

RE-5 CO ester

Zero-point correction= 0.541659 (Hartree/Particle)

Thermal correction to Energy=

Thermal correction to Enthalpy=
0.572913

0.573857 


\begin{tabular}{|c|c|c|c|c|}
\hline \multicolumn{5}{|c|}{ Thermal correction to Gibbs Free Energy= } \\
\hline & & -point Energies & & \\
\hline Sun & tronic and ther & mal Energies= & & \\
\hline & 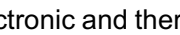 & $-e^{-}$ & & \\
\hline Sun & Thi and the & & - & \\
\hline \multicolumn{5}{|c|}{ Electronic energy (M06): -1440.568893 } \\
\hline $\mathrm{Pd}$ & -0.69872200 & -0.01661500 & & \\
\hline $\mathrm{P}$ & 1.74884300 & -0.07949000 & 0.01 & 1993400 \\
\hline C & -2.56440800 & 0.77740500 & -0.11 & 1389400 \\
\hline C & -3.00384100 & 1.54448200 & & $221 / 300$ \\
\hline C & -2.43819300 & 1.39557000 & & - \\
\hline C & -3.18529300 & 2.91518200 & & 7383800 \\
\hline $\mathrm{H}$ & -3.16520700 & 1.06590400 & -2.18 & 8383000 \\
\hline C & -2.62604200 & 2.77981000 & & 7330600 \\
\hline $\mathrm{H}$ & -2.26117300 & 0.78716200 & & 4134200 \\
\hline C & -2.99398600 & 3.54473200 & & 6707300 \\
\hline $\mathrm{H}$ & -3.48147700 & 3.50483300 & -1.93 & 3886400 \\
\hline $\mathrm{H}$ & -2.50726800 & 3.24968600 & & 4732000 \\
\hline $\mathrm{H}$ & -3.15136300 & 4.61473400 & & 6672800 \\
\hline $\mathrm{O}$ & -3.61134300 & -0.72355200 & & 8089500 \\
\hline C & 2.21611800 & -0.54278300 & & 5274600 \\
\hline C & 2.58153700 & -1.37742800 & -1.17 & 7216600 \\
\hline C & 2.49150000 & 325200 & -0.38 & 8042 \\
\hline C & -2.97993900 & 387700 & -0.34 & 4627 \\
\hline C & -1.77483900 & -1.69930600 & & 2470900 \\
\hline $\mathrm{H}$ & -1.91756200 & -1.14843700 & -2.06 & 6000500 \\
\hline $\mathrm{H}$ & -1.33728000 & -2.68060000 & -1.29 & 9952500 \\
\hline O & -3.47141400 & -2.95076900 & -0.04 & 4445800 \\
\hline C & -4.58892600 & -3.00461900 & & 5923700 \\
\hline $\mathrm{H}$ & 0800 & 68900 & & $8727 \varepsilon$ \\
\hline $\mathrm{H}$ & -4.37675100 & -2.43648300 & & 68721 \\
\hline $\mathrm{H}$ & -5.48627700 & -2.60062600 & & 8393200 \\
\hline C & 1.26981800 & -1.68384400 & & 0183900 \\
\hline $\mathrm{H}$ & 1.43395400 & -1.87929100 & & 7049500 \\
\hline $\mathrm{H}$ & 0.22116000 & -1.40203500 & & 6003300 \\
\hline $\mathrm{H}$ & 1.43668900 & -2.62018000 & & \\
\hline C & 7100 & 8900 & & 8203 \\
\hline $\mathrm{H}$ & 2.58865200 & 1.48429000 & & 6490500 \\
\hline $\mathrm{H}$ & 0.87432800 & 1.01702700 & & 3174700 \\
\hline $\mathrm{H}$ & 1.97348100 & 0.30917600 & & 2322300 \\
\hline C & 3.67630700 & -0.97105700 & & 0128200 \\
\hline $\mathrm{H}$ & 3.81802800 & -1.15620000 & & 7525300 \\
\hline $\mathrm{H}$ & 3.93566900 & -1.8 & & \\
\hline $\mathrm{H}$ & 4.35 & -0.2 & & 0570 \\
\hline C & 1.54393900 & 2.74097300 & 0.22 & 2793200 \\
\hline $\mathrm{H}$ & 0.51831100 & 2.61344100 & -0.13 & 3472500 \\
\hline $\mathrm{H}$ & 1.51676500 & 2.72702500 & & 1764600 \\
\hline $\mathrm{H}$ & 1.89048700 & 3.73749100 & -0.07 & 7825400 \\
\hline C & & 1.94186500 & 0.11 & 1257200 \\
\hline $\mathrm{H}$ & 4.24361800 & 2.942 & -0.21 & 1678 \\
\hline $\mathrm{H}$ & 4.00 & 1.92 & 1.20 & 0274 \\
\hline $\mathrm{H}$ & 4.65075200 & 1.22556000 & -0.28 & 8862500 \\
\hline C & 2.44873900 & 1.91648000 & -1.90 & 0656600 \\
\hline $\mathrm{H}$ & 2.68058600 & 2.97217500 & -2.10 & 0011200 \\
\hline $\mathrm{H}$ & 3.18389900 & 1.32255800 & -2.45 & 5416600 \\
\hline $\mathrm{H}$ & 1.45492500 & 1.71739600 & -2.32 & 2091500 \\
\hline C & 4.10418600 & -1.24403200 & -1.37 & 7424800 \\
\hline $\mathrm{H}$ & 4.45216400 & -2.06076300 & -2.02 & 2193800 \\
\hline $\mathrm{H}$ & 4.38014500 & -0.30817400 & -1.86 & 6692400 \\
\hline $\mathrm{H}$ & 4.66324500 & -1.31125800 & -0.43 & 3728500 \\
\hline C & 2.27681600 & -2.80141200 & -0.65 & 5554300 \\
\hline $\mathrm{H}$ & 2.57609000 & -3.52343000 & -1.42 & 2686100 \\
\hline $\mathrm{H}$ & 2.83036400 & -3.05553100 & & 5122600 \\
\hline $\mathrm{H}$ & 1.20798500 & -2.94234900 & -0.46 & 6662700 \\
\hline C & 1.87801600 & -1.27639900 & -2.54 & 4718900 \\
\hline $\mathrm{H}$ & 2.09039400 & -0.34639600 & -3.07 & 7490200 \\
\hline $\mathrm{H}$ & 2.22920500 & -2.10009400 & & 8351900 \\
\hline $\mathrm{H}$ & 0.79328900 & -1.36487800 & & 388940 \\
\hline
\end{tabular}

RE-3_CO_ketone $\begin{array}{lc}\text { Zero-point correction= } & 0.537385 \text { (Hartree/Particle) } \\ \text { Thermal correction to Energy= } & 0.567687 \\ \text { Thermal correction to Enthalpy= } & 0.568631 \\ \text { Thermal correction to Gibbs Free Energy= } & 0.478321 \\ \text { Sum of electronic and zero-point Energies }= & -1365.237689 \\ \text { Sum of electronic and thermal Energies= } & -1365.207387 \\ \text { Sum of electronic and thermal Enthalpies }= & -1365.206443 \\ \text { Sum of electronic and thermal Free Energies }= & -1365.296754\end{array}$

Electronic energy (M06): -1365.357236

\begin{tabular}{|c|c|c|c|}
\hline C & -1.09221500 & 0.60893600 & -0.16829400 \\
\hline C & -1.18607400 & 1.51790000 & -1.23107500 \\
\hline C & -1.45967700 & 0.97160000 & 1.13105500 \\
\hline C & -1.56434200 & 2.83323200 & -0.95965600 \\
\hline C & -1.84289700 & 2.29498300 & 1.37659300 \\
\hline C & -1.89065000 & 3.23220300 & 0.34209100 \\
\hline $\mathrm{H}$ & -1.61834000 & 3.54724000 & -1.77840800 \\
\hline $\mathrm{H}$ & -2.10885200 & 2.58673200 & 2.39022600 \\
\hline $\mathrm{P}$ & 2.49364400 & -0.00066600 & 0.12213400 \\
\hline C & 3.06423000 & 1.35432900 & -1.00140800 \\
\hline C & 3.95461200 & -1.14178100 & 0.12642200 \\
\hline $\mathrm{H}$ & -1.45117300 & 0.23599300 & 1.92900600 \\
\hline $\mathrm{H}$ & -2.19363600 & 4.25588900 & 0.54224500 \\
\hline $\mathrm{H}$ & -0.96380200 & 1.19759700 & -2.24367100 \\
\hline$P d$ & 0.41528400 & -0.83997100 & -0.35466400 \\
\hline C & -2.52369200 & -1.69176300 & 0.10982100 \\
\hline C & 2.62018600 & 0.79775500 & 1.78599000 \\
\hline C & -3.95963300 & -1.40278100 & -0.24732700 \\
\hline $\mathrm{H}$ & -4.15862100 & -0.32698300 & -0.16555200 \\
\hline $\mathrm{H}$ & -4.15753300 & -1.68983700 & -1.28694000 \\
\hline $\mathrm{H}$ & -4.65428800 & -1.94017200 & 0.40496700 \\
\hline C & -2.14758100 & -2.46742600 & 1.13997100 \\
\hline $\mathrm{H}$ & -2.88353800 & -2.95781600 & 1.76846300 \\
\hline $\mathrm{O}$ & -1.64856300 & -1.08309400 & -0.73759900 \\
\hline $\mathrm{H}$ & -1.09654500 & -2.63730800 & 1.35690400 \\
\hline C & 5.25438355 & -0.39801865 & 0.48560370 \\
\hline $\mathrm{H}$ & 6.07409711 & -1.08561869 & 0.47234251 \\
\hline $\mathrm{H}$ & 5.42941013 & 0.37943104 & -0.22842548 \\
\hline $\mathrm{H}$ & 5.16273103 & 0.02890142 & 1.46245530 \\
\hline C & 4.11191526 & -1.78424946 & -1.26429363 \\
\hline $\mathrm{H}$ & 5.11221356 & -2.14646242 & -1.37877109 \\
\hline $\mathrm{H}$ & 3.42449597 & -2.59851915 & -1.36083161 \\
\hline $\mathrm{H}$ & 3.90833133 & -1.05415701 & -2.01955425 \\
\hline C & 3.72398421 & -2.27053991 & 1.14833890 \\
\hline $\mathrm{H}$ & 3.04650471 & -2.99005378 & 0.73819096 \\
\hline $\mathrm{H}$ & 4.65643947 & -2.74544355 & 1.37170791 \\
\hline $\mathrm{H}$ & 3.30876709 & -1.86038960 & 2.04515073 \\
\hline C & 2.00532770 & 2.47229596 & -1.02322088 \\
\hline $\mathrm{H}$ & 1.27671446 & 2.25877467 & -1.77716479 \\
\hline $\mathrm{H}$ & 1.52515071 & 2.52742154 & -0.06860513 \\
\hline $\mathrm{H}$ & 2.47838745 & 3.40746062 & -1.23904844 \\
\hline C & 4.41366775 & 1.94126344 & -0.54739728 \\
\hline $\mathrm{H}$ & 5.21029085 & 1.43670375 & -1.05307618 \\
\hline $\mathrm{H}$ & 4.44606850 & 2.98457586 & -0.78266181 \\
\hline $\mathrm{H}$ & 4.52223985 & 1.81031564 & 0.50899505 \\
\hline C & 3.20796161 & 0.80750551 & -2.43386229 \\
\hline $\mathrm{H}$ & 2.85874280 & 1.53952230 & -3.13178609 \\
\hline $\mathrm{H}$ & 4.23679481 & 0.58925300 & -2.63075843 \\
\hline $\mathrm{H}$ & 2.62821262 & -0.08619447 & -2.53431860 \\
\hline C & 3.99348882 & 1.46167615 & 1.99776367 \\
\hline $\mathrm{H}$ & 3.90851294 & 2.51608592 & 1.83683163 \\
\hline $\mathrm{H}$ & 4.32740846 & 1.27928383 & 2.99782902 \\
\hline $\mathrm{H}$ & 4.69872260 & 1.05095432 & 1.30577169 \\
\hline C & 1.51217620 & 1.85880600 & 1.92047067 \\
\hline $\mathrm{H}$ & 0.69571774 & 1.45290664 & 2.48041824 \\
\hline $\mathrm{H}$ & 1.90028994 & 2.71752197 & 2.42729867 \\
\hline $\mathrm{H}$ & 1.17067027 & 2.14321284 & 0.94713288 \\
\hline C & 2.39742955 & -0.26192929 & 2.88100085 \\
\hline $\mathrm{H}$ & 3.34400753 & -0.61423564 & 3.23422934 \\
\hline $\mathrm{H}$ & 1.85320365 & 0.17322279 & 3.69301122 \\
\hline $\mathrm{H}$ & 1.84030516 & 1.08104888 & 2.47658088 \\
\hline
\end{tabular}




\section{RE-5 CO ketone}

Zero-point correction=

Thermal correction to Energy=

Thermal correction to Enthalpy=

0.535575 (Hartree/Particle)

0.566236

0.567180

0.474759

correction to Gibbs Free Energy=

mero-point Energies =

$-1365.231752$

$-1365.201091$

Sum of electronic and thermal Enthalpies $=\quad-1365.200147$

Sum of electronic and thermal Free Energies $=-1365.292568$

Electronic energy (M06): -1365.354199

c

H

$\mathrm{H}$

C

H

$\mathrm{H}$

C

$\mathrm{H}$

C

$\mathrm{H}$

$\mathrm{H}$

C

$\mathrm{H}$

$\mathrm{H}$

$\mathrm{H}$

C $\begin{array}{lll}-0.82873700 & -0.23373400 & -0.12592900\end{array}$

$\begin{array}{lll}1.61137100 & 0.05802500 & -0.00580500\end{array}$

$\begin{array}{llll}-2.78199800 & 0.30440600 & -0.02947000\end{array}$

$\begin{array}{lll}-3.37142300 & 0.86897400 & -1.18412900\end{array}$

$\begin{array}{lll}-2.75745200 & 1.04754100 & 1.17746700\end{array}$

$\begin{array}{lll}-3.81274300 & 2.18988300 & -1.15125400\end{array}$

$\begin{array}{lll}-3.45290100 & 0.28499300 & -2.09665600\end{array}$

$\begin{array}{lll}-3.19733400 & 2.37694400 & 1.17874000\end{array}$

$\begin{array}{lll}-2.45449800 & 0.56609800 & 2.10322400\end{array}$

$\begin{array}{lll}-3.72233900 & 2.95387000 & 0.02142000\end{array}$

$\begin{array}{lll}-4.23152700 & 2.63089200 & -2.05342100\end{array}$

$\begin{array}{lll}-3.15561400 & 2.94874500 & 2.10333500\end{array}$

$\begin{array}{lll}-4.07869300 & 3.97995100 & 0.03332500\end{array}$

$\begin{array}{lll}-3.57958900 & -1.38567900 & 0.39443600\end{array}$

$\begin{array}{lll}2.15841000 & 0.01869900 & 1.86370300\end{array}$

$2.61074100-1.31298100-0.96399700$

$2.08854500 \quad 1.79464900-0.75115600$

$\begin{array}{lll}-2.83660100 & -2.33864600 & 0.01377400\end{array}$

$\begin{array}{lll}-1.66815500 & -2.14825600 & -0.79536000\end{array}$

$-1.90108400-1.65722000-1.74880200$

$\begin{array}{lll}-1.06698300 & -3.04348800 & -0.95238700\end{array}$

$\begin{array}{llll}1.38905500 & -1.13725400 & 2.55017500\end{array}$

$1.59208500-1.10513800 \quad 3.62935900$

$\begin{array}{llll}0.30903500 & -1.03267400 & 2.40075400\end{array}$

$1.68150800 \quad-2.12471700 \quad 2.19259500$

$\begin{array}{lll}1.67925200 & 1.30720700 & 2.56965400\end{array}$

$\begin{array}{lll}2.24270800 & 2.19448200 & 2.27211600\end{array}$

$\begin{array}{lll}0.61408300 & 1.49380200 & 2.39797000\end{array}$

$\begin{array}{lll}1.82233400 & 1.18371500 & 3.65136800\end{array}$

$3.66702400-0.14824500 \quad 2.13385800$

$3.84246800-0.11043800 \quad 3.21793500$

$\begin{array}{lll}4.05212000 & -1.10856600 & 1.78259700\end{array}$

$\begin{array}{lll}4.26672300 & 0.64451500 & 1.67928500\end{array}$

$\begin{array}{llll}1.00642100 & 2.81089900 & -0.31070200\end{array}$

$\begin{array}{llll}0.00460000 & 2.47770400 & -0.60254100\end{array}$

$\begin{array}{lll}0.99689200 & 2.99772100 & 0.76305400\end{array}$

$\begin{array}{llll}1.20249800 & 3.77041100 & -0.80857900\end{array}$

$3.47865700 \quad 2.34432700-0.37211900$

$\begin{array}{llll}3.63998200 & 3.29917800 & -0.89173700\end{array}$

$\begin{array}{lll}3.57384500 & 2.54604800 & 0.69805200\end{array}$

$\begin{array}{llll}4.29101800 & 1.67437800 & -0.66478100\end{array}$

$\begin{array}{llll}1.99279100 & 1.73525400 & -2.29234300\end{array}$

$2.06225600 \quad 2.75876200 \quad-2.68379700$

$2.80219700 \quad 1.16276700 \quad-2.75116200$

$\begin{array}{lll}1.03500300 & 1.32150800 & -2.62459000\end{array}$

$4.09765500-1.00980800-1.23694300$

$\begin{array}{llll}4.55533600 & -1.87900900 & -1.72953600\end{array}$

$4.23380900-0.15695800-1.90700400$

$4.66542800-0.81690300-0.32316500$

$2.51496200-2.64371800-0.18438400$

$2.90230000-3.44978000-0.82166600$

$3.10968900-2.64805000 \quad 0.73198400$

$1.47858700-2.88925900 \quad 0.06795900$

$1.89518600-1.57171100-2.31221600$

$1.95323600-0.72985900-3.00196900$

$2.37341700-2.42976100-2.80401200$

$0.83946200-1.81134500-2.15838900$

$-3.27435600-3.72935200 \quad 0.42393400$

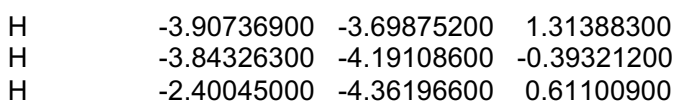

$\begin{array}{llrr}\mathrm{H} & -3.84326300 & -4.19108600 & -0.39321200 \\ \mathrm{H} & -2.40045000 & -4.36196600 & 0.61100900\end{array}$

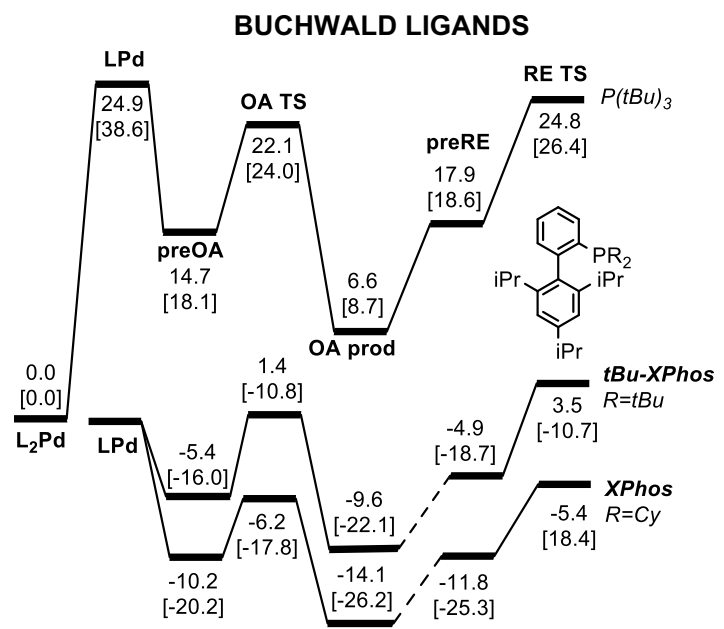

tBuXPhos_Pd

Zero-point correction $=$ 0.589517 (Hartree/Particle) Thermal correction to Enthalpy= $\quad 0.622944$

Thermal correction to Gibbs Free Energy $=\quad 0.529371$

Sum of electronic and zero-point Energies $=\quad-1481.779170$

Sum of electronic and thermal Energies $=\quad-1481.746686$

Sum of electronic and thermal Enthalpies $=\quad-1481.745742$

Sum of electronic and thermal Free Energies $=-1481.839316$ Electronic energy (M06): -1481.868123

$\begin{array}{llrr}\text { P } & 1.58341400 & -0.19836700 & -0.27847700 \\ \mathrm{C} & 0.59039900 & -0.84929800 & 1.18771300 \\ \mathrm{C} & 1.25054100 & -1.54506700 & 2.21797000 \\ \mathrm{C} & 0.59391300 & -2.01374600 & 3.35496500 \\ \mathrm{H} & 1.15044100 & -2.54373500 & 4.12344100 \\ \mathrm{C} & -0.77344200 & -1.79195300 & 3.49052100 \\ \mathrm{H} & -1.30837100 & -2.14031800 & 4.37007100 \\ \mathrm{C} & -1.45540200 & -1.11384100 & 2.48299900 \\ \mathrm{C} & -0.81770400 & -0.63677000 & 1.32335200 \\ \mathrm{C} & -1.67689900 & 0.06694900 & 0.30141800 \\ \mathrm{C} & -1.54031800 & 1.50400600 & 0.16223500 \\ \mathrm{C} & -1.07572200 & 2.35416200 & 1.35349700 \\ \mathrm{H} & -0.28955000 & 1.79996700 & 1.87521800 \\ \mathrm{C} & -0.50111300 & 3.72772600 & 0.97079500 \\ \mathrm{H} & -1.27086300 & 4.39475800 & 0.56423600 \\ \mathrm{H} & 0.29398700 & 3.63172400 & 0.22223900 \\ \mathrm{C} & -2.24751100 & 2.53482900 & 2.34383100 \\ \mathrm{H} & -2.62719200 & 1.57139300 & 2.69839900 \\ \mathrm{H} & -3.07859400 & 3.07028000 & 1.86908100 \\ \mathrm{C} & -2.18489900 & 2.12677700 & -0.93946800 \\ \mathrm{H} & -2.14800800 & 3.20691000 & -1.03973400 \\ \mathrm{C} & -3.05025600 & 1.39046200 & -1.77513400 \\ \mathrm{C} & -3.29147800 & 0.05391200 & -1.51793700 \\ \mathrm{H} & -4.00272700 & -0.48474100 & -2.13664300 \\ \mathrm{C} & -2.59691400 & -0.63900100 & -0.49685400 \\ \mathrm{C} & -2.89743300 & -2.12751100 & -0.30807100 \\ \mathrm{H} & -2.11149400 & -2.55762300 & 0.32114900 \\ \mathrm{C} & -4.23961700 & -2.32352400 & 0.43057700 \\ \mathrm{H} & -4.24220200 & -1.83215400 & 1.40893300 \\ \mathrm{H} & -5.06833500 & -1.90294200 & -0.15173500 \\ \mathrm{C} & -2.91980100 & -2.92596600 & -1.62724600 \\ \mathrm{H} & -3.78254200 & -2.66113800 & -2.24916800 \\ \mathrm{H} & -2.01696700 & -2.75729100 & -2.22067000 \\ \mathrm{H} & -1.92572000 & 3.11397300 & 3.21815900 \\ & -0.08559800 & 4.22004600 & 1.85807600\end{array}$




$\begin{array}{lrrr}\mathrm{H} & -2.99367100 & -3.99926800 & -1.41398100 \\ \mathrm{H} & -4.44442500 & -3.39026200 & 0.58538800 \\ \mathrm{C} & 2.05243500 & -1.80144800 & -1.24820900 \\ \mathrm{C} & 2.99843400 & -1.41348100 & -2.40152000 \\ \mathrm{C} & 2.66267700 & -2.95995900 & -0.43672400 \\ \mathrm{C} & 0.72673000 & -2.30067800 & -1.85647000 \\ \mathrm{H} & 3.99637100 & -1.13340100 & -2.04987200 \\ \mathrm{H} & 2.59311700 & -0.58007300 & -2.98620300 \\ \mathrm{H} & 1.98542800 & -3.30639400 & 0.34944200 \\ \mathrm{H} & 3.62095900 & -2.70639400 & 0.02203400 \\ \mathrm{H} & 0.24653000 & -1.52697300 & -2.46480100 \\ \mathrm{H} & 0.02260300 & -2.60658600 & -1.07683800 \\ \mathrm{C} & 3.18060000 & 0.62266700 & 0.47128400 \\ \mathrm{C} & 2.76309800 & 1.46107000 & 1.69660500 \\ \mathrm{C} & 4.35233700 & -0.29436900 & 0.87290400 \\ \mathrm{C} & 3.69347800 & 1.60026000 & -0.61251900 \\ \mathrm{H} & 2.43178900 & 0.84196300 & 2.53510400 \\ \mathrm{H} & 1.95884600 & 2.16035400 & 1.44909100 \\ \mathrm{H} & 4.74871200 & -0.86105000 & 0.02597500 \\ \mathrm{H} & 4.09761900 & -0.99968300 & 1.66871900 \\ \mathrm{H} & 2.91129900 & 2.30727200 & -0.90668000 \\ \mathrm{H} & 4.03058700 & 1.08124700 & -1.51494600 \\ \mathrm{H} & 4.54750000 & 2.16646700 & -0.21590500 \\ \mathrm{H} & 5.17282200 & 0.32939300 & 1.25357700 \\ \mathrm{H} & 3.62374700 & 2.05331600 & 2.03542600 \\ \mathrm{H} & 3.11965300 & -2.27170300 & -3.07585100 \\ \mathrm{H} & 0.92086300 & -3.17474000 & -2.49299200 \\ \mathrm{H} & 2.84000000 & -3.81004800 & -1.11008200 \\ \mathrm{H} & -3.56301200 & 1.89396700 & -2.59113100 \\ \mathrm{H} & -2.52154000 & -0.93906600 & 2.58978200 \\ \mathrm{H} & 2.31428600 & -1.72826200 & 2.13443400 \\ \mathrm{Pd} & 0.12012300 & 1.27845800 & -1.30318900\end{array}$

\section{tBuXPhos_preOA}

Zero-point correction=

Thermal correction to Enthalpy=

0.681212 (Hartree/Particle)

Thermal correction to Gibbs Free Energy $=\quad 0.608136$

Sum of electronic and zero-point Energies $=\quad-1726.504114$

Sum of electronic and thermal Energies $=\quad-1726.463699$

Sum of electronic and thermal Enthalpies $=\quad-1726.462755$

Sum of electronic and thermal Free Energies $=-1726.577190$

\section{Electronic energy (M06): -1726.555156}

$\begin{array}{lrrr}\mathrm{P} & 1.04983200 & 1.50415700 & -0.34002200 \\ \mathrm{C} & 2.53063400 & 0.36550000 & -0.12582000 \\ \mathrm{C} & 3.82590600 & 0.90825700 & -0.26081300 \\ \mathrm{C} & 4.98688200 & 0.16019000 & -0.08534400 \\ \mathrm{H} & 5.95899100 & 0.63218100 & -0.20048300 \\ \mathrm{C} & 4.88149000 & -1.18928900 & 0.23981100 \\ \mathrm{H} & 5.76920000 & -1.79910000 & 0.38514600 \\ \mathrm{C} & 3.61732600 & -1.75178700 & 0.38196900 \\ \mathrm{C} & 2.42708800 & -1.01458400 & 0.20964500 \\ \mathrm{C} & 1.17475700 & -1.83836500 & 0.42996500 \\ \mathrm{C} & 0.67645600 & -2.01321800 & 1.74674100 \\ \mathrm{C} & 1.27980500 & -1.29399100 & 2.95205000 \\ \mathrm{H} & 1.90990900 & -0.48329200 & 2.57769500 \\ \mathrm{C} & 0.21508100 & -0.66388300 & 3.86953500 \\ \mathrm{H} & -0.38004500 & -1.42661200 & 4.38621000 \\ \mathrm{H} & -0.47420800 & -0.02895800 & 3.30353800 \\ \mathrm{C} & 2.18880400 & -2.24243300 & 3.76173400 \\ \mathrm{H} & 3.00483200 & -2.63737100 & 3.14751500 \\ \mathrm{H} & 1.61906500 & -3.09502500 & 4.15067800 \\ \mathrm{C} & -0.35395100 & -2.93606800 & 1.96731900 \\ \mathrm{H} & -0.73137600 & -3.08092900 & 2.97579400 \\ \mathrm{C} & -0.88853800 & -3.68578400 & 0.92482800 \\ \mathrm{C} & -0.38130600 & -3.52960200 & -0.36056700 \\ \mathrm{H} & -0.78637000 & -4.13228900 & -1.16751700 \\ \mathrm{C} & 0.65693500 & -2.62865200 & -0.62948400 \\ \mathrm{C} & 1.25373900 & -2.60394900 & -2.03649700 \\ \mathrm{H} & 1.85922100 & -1.69721200 & -2.12709700\end{array}$

$2.19869100-3.80537300 \quad-2.25250100$

$3.02057000-3.80779800-1.52955200$

$\begin{array}{lll}1.65538500 & -4.75226800 & -2.14767700\end{array}$

$0.18889300-2.56740200-3.14840700$

$\begin{array}{lll}-0.35088200 & -3.51848300 & -3.22460400\end{array}$

$-0.54850700-1.77891500-2.97548800$

$\begin{array}{lll}2.63241200 & -1.71562900 & 4.61568200\end{array}$

$0.69665300 \quad-0.05288400 \quad 4.64274800$

$0.66646400-2.39024300-4.11958100$

$2.63378700-3.77602800-3.25917100$

$\begin{array}{lll}-1.68433100 & -4.40178200 & 1.11515400\end{array}$

$\begin{array}{lll}3.53102200 & -2.80260800 & 0.64265100\end{array}$

$\begin{array}{lll}3.93553900 & 1.95408400 & -0.51078500\end{array}$

$\begin{array}{lll}-1.11786700 & 0.61123100 & -0.06389100\end{array}$

$\begin{array}{lll}-3.19912900 & 0.14465400 & 0.07653700\end{array}$

$\begin{array}{lll}-3.46341400 & 1.53210200 & -0.05179300\end{array}$

$\begin{array}{lll}-3.21838600 & -0.47413700 & 1.35036400\end{array}$

$\begin{array}{lll}-3.77852700 & 2.27323700 & 1.10140400\end{array}$

$\begin{array}{lll}-3.55107500 & 1.97841900 & -1.03575400\end{array}$

$\begin{array}{lll}-3.51662800 & 0.29586900 & 2.47427700\end{array}$

$\begin{array}{lll}-3.03005200 & -1.53837900 & 1.43273100\end{array}$

$\begin{array}{lll}-3.82007500 & 1.65997500 & 2.35080200\end{array}$

$\begin{array}{lll}-4.01299600 & 3.32974500 & 1.00034300\end{array}$

$\begin{array}{lll}-3.52845100 & -0.17868900 & 3.45185700\end{array}$

$\begin{array}{lll}-4.08539500 & 2.23781700 & 3.23165200\end{array}$

$\begin{array}{lll}-3.47958400 & -1.02184600 & -1.55026700\end{array}$

$\begin{array}{lll}0.02595900 & 3.68104200 & 1.13547500\end{array}$

$\begin{array}{lll}-0.34865700 & 4.18010700 & 0.23780700\end{array}$

$\begin{array}{lll}-0.73955200 & 2.97285700 & 1.46601700\end{array}$

$\begin{array}{lll}0.15396400 & 4.44754200 & 1.91244700\end{array}$

$\begin{array}{llll}2.40965700 & 4.04558900 & 0.48308900\end{array}$

$\begin{array}{llll}2.45899900 & 4.80719900 & 1.27333100\end{array}$

$\begin{array}{llll}3.42111300 & 3.64713400 & 0.36838200\end{array}$

$\begin{array}{llll}2.13341200 & 4.56164900 & -0.43992700\end{array}$

$\begin{array}{lll}1.38072800 & 2.97570200 & 0.89810900\end{array}$

$\begin{array}{lll}1.82627200 & 2.36373500 & 2.23994600\end{array}$

$\begin{array}{lll}1.09900000 & 1.63380000 & 2.60463700\end{array}$

$\begin{array}{lll}2.80331300 & 1.87635000 & 2.17127500\end{array}$

$\begin{array}{lll}1.90002900 & 3.16131300 & 2.99141500\end{array}$

$\begin{array}{lll}1.18213500 & 2.16193700 & -2.16154900\end{array}$

$\begin{array}{lll}2.56522900 & 2.61145100 & -2.67028200\end{array}$

$\begin{array}{llll}3.27947700 & 1.78374500 & -2.69213700\end{array}$

$\begin{array}{lll}2.45784100 & 2.97333300 & -3.70197700\end{array}$

$\begin{array}{llll}2.99855800 & 3.42765200 & -2.08689500\end{array}$

$\begin{array}{lll}0.17650500 & 3.32112600 & -2.32952700\end{array}$

$\begin{array}{llll}0.07354400 & 3.55122000 & -3.39839600\end{array}$

$\begin{array}{lll}-0.81338900 & 3.05045900 & -1.94638300\end{array}$

$\begin{array}{llll}0.50179900 & 4.23922500 & -1.83142400\end{array}$

$\begin{array}{llll}0.71170600 & 1.00227300 & -3.06226800\end{array}$

$\begin{array}{lll}1.39171900 & 0.14739800 & -3.01023800\end{array}$

$\begin{array}{lll}-0.29120100 & 0.66113400 & -2.78641400\end{array}$

$\begin{array}{llll}0.68617700 & 1.34102100 & -4.10705700\end{array}$

\section{tBuXPhos_OA_TS}

Zero-point correction=

Thermal correction to Enthalpy= $\quad 0.722054$

Thermal correction to Gibbs Free Energy $=\quad 0.610285$

Sum of electronic and zero-point Energies $=\quad-1726.499812$

Sum of electronic and thermal Energies $=\quad-1726.460301$

Sum of electronic and thermal Enthalpies $=\quad-1726.459357$

Sum of electronic and thermal Free Energies $=-1726.571126$

Electronic energy (M06): -1726.546387

$\begin{array}{llll}\mathrm{P} & -0.96596200 & 1.56596600 & -0.16635700\end{array}$

C $\quad-2.50564400 \quad 0.49387500 \quad-0.22232500$

C $\quad \begin{array}{llll}\text { C } & -3.75218700 & 1.13161400 & -0.39689800\end{array}$

$\begin{array}{lllll}\text { C } & -4.95660900 & 0.43851300 & -0.47848100\end{array}$

$\mathrm{H} \quad-5.88761500 \quad 0.98219200 \quad-0.61453000$

$\begin{array}{llll}\text { C } & -4.94665200 & -0.95040200 & -0.38551100\end{array}$ 


$\begin{array}{llll}\mathrm{H} & -1.76942600 & 1.08737100 & 2.70631400 \\ \mathrm{H} & -0.01748600 & 1.36395800 & 2.59732300 \\ \mathrm{H} & -1.04613700 & 2.48564300 & 3.50816900\end{array}$

tBuXPhos_OA_TS_prod

Zero-point correction=

Thermal correction to Energy=

0.683118 (Hartree/Particle)

correction to Enthalpy=

Thermal correction to Gibbs Free Energy $=\quad 0.612522$

Sum of electronic and zero-point Energies $=\quad-1726.518822$

Sum of electronic and thermal Energies $=\quad-1726.478987$

Sum of electronic and thermal Enthalpies $=\quad-1726.478043$

Sum of electronic and thermal Free Energies $=-1726.589418$ Electronic energy (M06): -1726.566163

H

$\mathrm{H}$

$\mathrm{H}$

$\mathrm{Pd}$

c

c

$\mathrm{C}$

H

H

H

C

$\mathrm{C}$

H
$1.80636400 \quad-0.80854200 \quad-0.02038300$

$\begin{array}{llll}2.35493000 & 0.97054300 & 0.00412200\end{array}$

$\begin{array}{llll}3.72017900 & 1.32325200 & -0.01953700\end{array}$

$\begin{array}{llll}4.14947100 & 2.64692800 & -0.04628100\end{array}$

$\begin{array}{llll}5.21212300 & 2.87269600 & -0.06471200\end{array}$

$3.20386500 \quad 3.67056500 \quad-0.05037600$

$\begin{array}{llll}3.51322500 & 4.71196900 & -0.07281500\end{array}$

$\begin{array}{lll}1.85107400 & 3.34682400 & -0.02835300\end{array}$

$\begin{array}{lll}1.39485600 & 2.01274500 & -0.00229400\end{array}$

$\begin{array}{lll}-0.12135000 & 1.89649100 & 0.00621900\end{array}$

$\begin{array}{lll}-0.82626100 & 2.04135600 & -1.23275600\end{array}$

$\begin{array}{lll}-0.12043900 & 1.98701700 & -2.58705700\end{array}$

$\begin{array}{llll}0.90415600 & 1.64772000 & -2.42170300\end{array}$

$\begin{array}{lll}-0.78668300 & 1.00540400 & -3.56807600\end{array}$

$\begin{array}{lll}-1.80640100 & 1.31418700 & -3.82454800\end{array}$

$\begin{array}{lll}-0.84213700 & -0.00304500 & -3.14492200\end{array}$

$\begin{array}{llll}-0.02989000 & 3.39503600 & -3.21384000\end{array}$

$\begin{array}{llll}0.49640300 & 4.09357700 & -2.55498400\end{array}$

$\begin{array}{llll}-1.02530100 & 3.80742800 & -3.41516600\end{array}$

$\begin{array}{lll}-2.18165300 & 2.39712400 & -1.21001000\end{array}$

$\begin{array}{lll}-2.71708500 & 2.50631200 & -2.14775400\end{array}$

$\begin{array}{lll}-2.84442300 & 2.64283500 & -0.01612200\end{array}$

$\begin{array}{lll}-2.16381700 & 2.51629200 & 1.19125100\end{array}$

$\begin{array}{lll}-0.81450600 & 2.15830000 & 1.23475300\end{array}$

$\begin{array}{lll}-0.10226700 & 2.18740800 & 2.58687000\end{array}$

$\begin{array}{lll}0.90503600 & 1.78597000 & 2.44566800\end{array}$

$\begin{array}{llll}0.05401000 & 3.63467700 & 3.10051800\end{array}$

$\begin{array}{lll}0.62042600 & 4.25368100 & 2.39759700\end{array}$

$\begin{array}{lll}-0.92351500 & 4.10655600 & 3.25370800\end{array}$

$\begin{array}{lll}-0.80776900 & 1.32221400 & 3.64781500\end{array}$

$\begin{array}{lll}-1.79647300 & 1.71997900 & 3.90353000\end{array}$

$\begin{array}{lll}-0.93767700 & 0.29281500 & 3.30245000\end{array}$

$\begin{array}{llll}0.51451000 & 3.35243900 & -4.16480900\end{array}$

$\begin{array}{lll}-0.21378300 & 0.95621500 & -4.50161800\end{array}$

$\begin{array}{lll}-0.21629500 & 1.30067200 & 4.57071800\end{array}$

$\begin{array}{llll}0.58460800 & 3.64190700 & 4.06018500\end{array}$

$\begin{array}{lll}-3.89302700 & 2.92501600 & -0.02264300\end{array}$

$\begin{array}{llll}1.11314500 & 4.14375300 & -0.03639600\end{array}$

$\begin{array}{llll}4.47088600 & 0.54445300 & -0.01501300\end{array}$

$\begin{array}{lll}-0.71166400 & -0.73160500 & -0.04333700\end{array}$

$\begin{array}{lll}-2.73387700 & -0.66981700 & -0.06177300\end{array}$

$\begin{array}{lll}-3.42112800 & -0.72085900 & -1.27827200\end{array}$

$\begin{array}{lll}-3.45173500 & -0.52987000 & 1.12918700\end{array}$

$\begin{array}{lll}-4.80972000 & -0.54968900 & -1.30808400\end{array}$

$\begin{array}{lll}-2.88691900 & -0.89473100 & -2.20774300\end{array}$

$\begin{array}{lll}-4.84109100 & -0.36224100 & 1.09710000\end{array}$

$\begin{array}{lll}-5.52247400 & -0.36420000 & -0.12148600\end{array}$

$\begin{array}{lll}-5.33374000 & -0.57820100 & -2.26124700\end{array}$

$\begin{array}{lll}-5.38972500 & -0.24696800 & 2.02964600\end{array}$

$-6.60287900-0.24488200-0.14502500$

$\begin{array}{lll}-1.15973200 & -3.23016100 & 0.19186000\end{array}$

$\begin{array}{lll}1.92328000 & -2.89990200 & -1.91628200\end{array}$

$\begin{array}{lll}2.04176600 & -3.67091800 & -1.15275200\end{array}$

$0.85094600-2.73450500-2.04447100$ $\begin{array}{lll}-2.68943400 & 2.72139900 & 2.11885100\end{array}$

$\begin{array}{lll}-2.94017600 & -0.55592600 & 2.08682600\end{array}$ 


$\begin{array}{lrrr}\mathrm{H} & 2.32837000 & -3.29705800 & -2.85696600 \\ \mathrm{C} & 4.17414500 & -1.90227000 & -1.43328500 \\ \mathrm{H} & 4.51936800 & -2.36268600 & -2.36872300 \\ \mathrm{H} & 4.77989500 & -1.00488300 & -1.27963000 \\ \mathrm{H} & 4.39046600 & -2.60934500 & -0.62959400 \\ \mathrm{C} & 2.67019800 & -1.59227100 & -1.57141800 \\ \mathrm{C} & 2.48778000 & -0.62785100 & -2.75907100 \\ \mathrm{H} & 1.43117900 & -0.44353900 & -2.96594900 \\ \mathrm{H} & 2.98961200 & 0.33186600 & -2.60282900 \\ \mathrm{H} & 2.91974600 & -1.08883300 & -3.65702300 \\ \mathrm{C} & 2.52194600 & -1.61366800 & 1.59104700 \\ \mathrm{C} & 3.95328900 & -1.19383900 & 1.97828000 \\ \mathrm{H} & 4.02761100 & -0.12235000 & 2.18442500 \\ \mathrm{H} & 4.22955800 & -1.72303800 & 2.90002400 \\ \mathrm{H} & 4.69823200 & -1.45548000 & 1.22222600 \\ \mathrm{C} & 2.45646000 & -3.15198900 & 1.47850100 \\ \mathrm{H} & 2.69233500 & -3.57877100 & 2.46249100 \\ \mathrm{H} & 1.46055700 & -3.50097300 & 1.19289200 \\ \mathrm{H} & 3.18737100 & -3.55379500 & 0.77163900 \\ \mathrm{C} & 1.58074000 & -1.18659500 & 2.73450500 \\ \mathrm{H} & 1.59754500 & -0.10436200 & 2.88994800 \\ \mathrm{H} & 0.55004900 & -1.50074200 & 2.54684100 \\ \mathrm{H} & 1.91429500 & -1.65770700 & 3.66877800\end{array}$

\section{tBuXPhos_preRE}

Zero-point correction=

Thermal correction to Energy=

Thermal correction to Enthalpy=

0.722640 (Hartree/Particle) 0.765062

0.766006

Thermal correction to Gibbs Free Energy $=\quad 0.649649$

Sum of electronic and zero-point Energies $=\quad-1957.679473$

Sum of electronic and thermal Energies $=\quad-1957.637050$

Sum of electronic and thermal Enthalpies $=\quad-1957.636106$

Sum of electronic and thermal Free Energies $=-1957.752463$

Electronic energy (M06): -1957.739242

$\begin{array}{lrrr}\mathrm{P} & 0.03495700 & 1.65460800 & -0.36124300 \\ \mathrm{C} & 1.90135300 & 1.54878900 & -0.25340000 \\ \mathrm{C} & 2.68109400 & 2.69468700 & -0.51607800 \\ \mathrm{C} & 4.06437900 & 2.71751700 & -0.36495200 \\ \mathrm{H} & 4.61768800 & 3.62786400 & -0.57844100 \\ \mathrm{C} & 4.72089700 & 1.56739600 & 0.06587600 \\ \mathrm{H} & 5.79854000 & 1.55956200 & 0.20434200 \\ \mathrm{C} & 3.97788100 & 0.41771600 & 0.31148400 \\ \mathrm{C} & 2.57728900 & 0.36859200 & 0.15194700 \\ \mathrm{C} & 1.99397100 & -1.00089300 & 0.44061400 \\ \mathrm{C} & 1.69103000 & -1.36357800 & 1.78684600 \\ \mathrm{C} & 1.70144900 & -0.34751500 & 2.92694600 \\ \mathrm{H} & 1.76882700 & 0.65035300 & 2.48746800 \\ \mathrm{C} & 0.41942300 & -0.39695500 & 3.77781700 \\ \mathrm{H} & 0.31987500 & -1.34919700 & 4.31131700 \\ \mathrm{H} & -0.47026800 & -0.26965300 & 3.15156000 \\ \mathrm{C} & 2.95080800 & -0.53322800 & 3.81398400 \\ \mathrm{H} & 3.87304500 & -0.43358300 & 3.23160800 \\ \mathrm{H} & 2.95869100 & -1.52106900 & 4.28936400 \\ \mathrm{C} & 1.50191600 & -2.71552400 & 2.10331600 \\ \mathrm{H} & 1.28226700 & -2.99596900 & 3.12918100 \\ \mathrm{C} & 1.63091500 & -3.70727700 & 1.13710400 \\ \mathrm{C} & 1.93108500 & -3.35683700 & -0.17622000 \\ \mathrm{H} & 2.03431200 & -4.13680000 & -0.92400800 \\ \mathrm{C} & 2.12341000 & -2.02105200 & -0.54975600 \\ \mathrm{C} & 2.56594500 & -1.73026200 & -1.98420900 \\ \mathrm{H} & 2.48184600 & -0.65162900 & -2.14812000 \\ \mathrm{C} & 4.04788400 & -2.10791200 & -2.19617700 \\ \mathrm{H} & 4.71227000 & -1.55038900 & -1.52889000 \\ \mathrm{H} & 4.20704100 & -3.17765400 & -2.01617300 \\ \mathrm{C} & 1.68834300 & -2.44311100 & -3.03257900 \\ \mathrm{H} & 1.88678400 & -3.52142700 & -3.05230300 \\ \mathrm{H} & 0.62128700 & -2.30991300 & -2.83781000 \\ \mathrm{H} & 2.96972500 & 0.22223900 & 4.60858400 \\ \mathrm{H} & 0.43324200 & 0.39978000 & 4.53115400\end{array}$

\begin{tabular}{|c|c|c|c|}
\hline $\mathrm{H}$ & 1.91758500 & -2.05761000 & -4.03314400 \\
\hline $\mathrm{H}$ & 4.34856000 & -1.89357100 & -3.22874900 \\
\hline C & -0.34777000 & 2.02660800 & -2.22293000 \\
\hline C & -1.80425400 & 2.51724300 & -2.35473300 \\
\hline C & 0.58511400 & 3.03564500 & -2.92034000 \\
\hline C & -0.21784600 & 0.68165900 & -2.96693000 \\
\hline $\mathrm{H}$ & -1.93709900 & 3.53781300 & -1.98434300 \\
\hline $\mathrm{H}$ & -2.51063600 & 1.86598400 & -1.83469500 \\
\hline $\mathrm{H}$ & 1.62061700 & 2.68594200 & -2.95166800 \\
\hline $\mathrm{H}$ & 0.56723600 & 4.02859800 & -2.46323200 \\
\hline $\mathrm{H}$ & -0.86615000 & -0.09567700 & -2.55058200 \\
\hline $\mathrm{H}$ & 0.81063100 & 0.31122300 & -2.94916800 \\
\hline C & -0.43429600 & 3.19671800 & 0.73154400 \\
\hline C & 0.30789300 & 3.05777900 & 2.07591800 \\
\hline C & -0.12700900 & 4.59099500 & 0.14657300 \\
\hline C & -1.94810100 & 3.13137700 & 1.02865200 \\
\hline $\mathrm{H}$ & 1.39426100 & 3.12544400 & 1.96595500 \\
\hline $\mathrm{H}$ & 0.06455600 & 2.11432000 & 2.57214100 \\
\hline $\mathrm{H}$ & -0.63451700 & 4.77419300 & -0.80334000 \\
\hline $\mathrm{H}$ & 0.94083700 & 4.77832100 & 0.01338800 \\
\hline $\mathrm{H}$ & -2.22845100 & 2.20375900 & 1.52826900 \\
\hline $\mathrm{H}$ & -2.56351200 & 3.22275300 & 0.13126200 \\
\hline $\mathrm{H}$ & -2.20780000 & 3.96634400 & 1.69328300 \\
\hline $\mathrm{H}$ & -0.49210200 & 5.34604200 & 0.85539000 \\
\hline $\mathrm{H}$ & -0.01180100 & 3.86994900 & 2.74191500 \\
\hline $\mathrm{H}$ & -2.07620700 & 2.52205000 & -3.41838000 \\
\hline $\mathrm{H}$ & -0.49409100 & 0.82675400 & -4.01987200 \\
\hline $\mathrm{H}$ & 0.24960100 & 3.15290500 & -3.95934000 \\
\hline $\mathrm{H}$ & 1.50185700 & -4.75232900 & 1.40650600 \\
\hline $\mathrm{H}$ & 4.48696700 & -0.48377100 & 0.63921600 \\
\hline $\mathrm{H}$ & 2.19590700 & 3.59923700 & -0.85281200 \\
\hline $\mathrm{Pd}$ & -0.88298800 & -0.59687100 & 0.16811700 \\
\hline C & -2.85460300 & -0.21750200 & 0.38880100 \\
\hline C & -3.72048700 & -0.12254700 & -0.70613400 \\
\hline C & -3.37428800 & -0.05330900 & 1.68051400 \\
\hline C & -5.07112600 & 0.19294600 & -0.51619000 \\
\hline $\mathrm{H}$ & -3.36118400 & -0.31770300 & -1.71277300 \\
\hline C & -4.72566300 & 0.25889600 & 1.86660600 \\
\hline $\mathrm{H}$ & -2.73400900 & -0.16382900 & 2.55191000 \\
\hline C & -5.57721400 & 0.39238200 & 0.76850300 \\
\hline $\mathrm{H}$ & -5.72822400 & 0.26452200 & -1.37990100 \\
\hline $\mathrm{H}$ & -5.11047100 & 0.38848800 & 2.87596600 \\
\hline $\mathrm{H}$ & -6.62773600 & 0.62986700 & 0.91435100 \\
\hline $\mathrm{O}$ & -3.45630600 & -3.27986300 & -0.88128200 \\
\hline $\mathrm{N}$ & -2.27877200 & -2.93510100 & -0.97583500 \\
\hline C & -1.54474400 & -2.58032800 & 0.24659800 \\
\hline 0 & -1.67902100 & -2.84843600 & -2.06125400 \\
\hline $\mathrm{H}$ & -2.16807400 & -2.84897500 & 1.09574300 \\
\hline $\mathrm{H}$ & -0.60267100 & -3.12921300 & 0.22014800 \\
\hline
\end{tabular}

tBuXPhos RE TS

Zero-point correction= 0.721305 (Hartree/Particle)

Thermal correction to Energy= $\quad 0.763294$

Thermal correction to Enthalpy= $\quad 0.764238$

Thermal correction to Gibbs Free Energy= $\quad 0.648341$

Sum of electronic and zero-point Energies $=\quad-1957.659591$

Sum of electronic and thermal Energies $=\quad-1957.617601$

Sum of electronic and thermal Enthalpies $=\quad-1957.616657$

Sum of electronic and thermal Free Energies $=-1957.732554$

Electronic energy (M06): -1957.724691

$\begin{array}{llrl}\mathrm{P} & 0.28072300 & 1.63963400 & -0.35761800 \\ \mathrm{C} & 2.12876600 & 1.34598500 & -0.24113700 \\ \mathrm{C} & 3.01526700 & 2.41771300 & -0.47376800 \\ \mathrm{C} & 4.39698000 & 2.29633800 & -0.35294600 \\ \mathrm{H} & 5.03558600 & 3.15489700 & -0.54215100 \\ \mathrm{C} & 4.94184700 & 1.06895400 & 0.01507500 \\ \mathrm{H} & 6.01620300 & 0.94653000 & 0.12258200 \\ \mathrm{C} & 4.09082000 & -0.00720300 & 0.24638900 \\ \mathrm{C} & 2.68961600 & 0.09040200 & 0.12505600\end{array}$




\begin{tabular}{|c|c|c|c|}
\hline C & 1.96256700 & -1.20724100 & 0.41943700 \\
\hline C & 1.61448900 & -1.53019000 & 1.76110200 \\
\hline C & 1.83098600 & -0.55822000 & 2.91978200 \\
\hline $\mathrm{H}$ & 2.03405700 & 0.42953400 & 2.49883100 \\
\hline C & 0.59687200 & -0.42860800 & 3.83225300 \\
\hline $\mathrm{H}$ & 0.38810200 & -1.35921300 & 4.37266500 \\
\hline $\mathrm{H}$ & -0.29637400 & -0.16781500 & 3.25345600 \\
\hline C & 3.07124600 & -0.96158000 & 3.74479600 \\
\hline $\mathrm{H}$ & 3.97241700 & -0.99012400 & 3.12320100 \\
\hline $\mathrm{H}$ & 2.94189000 & -1.95346000 & 4.19390300 \\
\hline C & 1.14563500 & -2.81783700 & 2.05241900 \\
\hline $\mathrm{H}$ & 0.88376800 & -3.07109200 & 3.07561500 \\
\hline C & 1.03083900 & -3.78719900 & 1.06056800 \\
\hline C & 1.40452200 & -3.48062700 & -0.24450500 \\
\hline $\mathrm{H}$ & 1.33612900 & -4.24699800 & -1.01025900 \\
\hline C & 1.88539100 & -2.21001400 & -0.58698400 \\
\hline C & 2.37728300 & -1.98609000 & -2.01668500 \\
\hline $\mathrm{H}$ & 2.48516900 & -0.90825200 & -2.16990300 \\
\hline C & 3.77146900 & -2.61558700 & -2.22545700 \\
\hline $\mathrm{H}$ & 4.51530600 & -2.19277100 & -1.54268400 \\
\hline $\mathrm{H}$ & 3.73891500 & -3.69914900 & -2.06001700 \\
\hline C & 1.39524400 & -2.51925400 & -3.07856100 \\
\hline $\mathrm{H}$ & 1.39926200 & -3.61494400 & -3.11605800 \\
\hline $\mathrm{H}$ & 0.36859800 & -2.19807800 & -2.88341100 \\
\hline $\mathrm{H}$ & 3.24191200 & -0.24365800 & 4.55613800 \\
\hline $\mathrm{H}$ & 0.76234100 & 0.35193500 & 4.58462300 \\
\hline $\mathrm{H}$ & 1.69037300 & -2.16442800 & -4.07308000 \\
\hline $\mathrm{H}$ & 4.11772000 & -2.44257700 & -3.25166500 \\
\hline C & -0.05014100 & 2.11365800 & -2.20548500 \\
\hline C & -1.48650700 & 2.66494200 & -2.32657900 \\
\hline C & 0.93269000 & 3.10532000 & -2.85661400 \\
\hline C & 0.02177400 & 0.79432300 & -3.00044500 \\
\hline $\mathrm{H}$ & -1.58853300 & 3.66961900 & -1.90644600 \\
\hline $\mathrm{H}$ & -2.21977600 & 2.01237500 & -1.84367700 \\
\hline $\mathrm{H}$ & 1.95120400 & 2.70845500 & -2.89056000 \\
\hline $\mathrm{H}$ & 0.95595100 & 4.07988000 & -2.36239500 \\
\hline $\mathrm{H}$ & -0.67909300 & 0.04508200 & -2.61900600 \\
\hline $\mathrm{H}$ & 1.02758800 & 0.36656200 & -2.97657100 \\
\hline C & -0.06566700 & 3.17490300 & 0.78216100 \\
\hline C & 0.69200600 & 2.95863300 & 2.10645700 \\
\hline C & 0.30403700 & 4.56554800 & 0.22777400 \\
\hline C & -1.57657100 & 3.16962500 & 1.10640700 \\
\hline $\mathrm{H}$ & 1.77834900 & 2.98525200 & 1.97938000 \\
\hline $\mathrm{H}$ & 0.41811000 & 2.00663300 & 2.56820200 \\
\hline $\mathrm{H}$ & -0.23418900 & 4.81137500 & -0.69086100 \\
\hline $\mathrm{H}$ & 1.37511900 & 4.68397400 & 0.04402600 \\
\hline $\mathrm{H}$ & -1.88812000 & 2.22766600 & 1.56363600 \\
\hline $\mathrm{H}$ & -2.20103600 & 3.33057100 & 0.22456700 \\
\hline $\mathrm{H}$ & -1.78875500 & 3.98243800 & 1.81432400 \\
\hline $\mathrm{H}$ & 0.02600300 & 5.32144800 & 0.97463400 \\
\hline $\mathrm{H}$ & 0.41697300 & 3.75669900 & 2.80867900 \\
\hline $\mathrm{H}$ & -1.74761300 & 2.73209300 & -3.39096000 \\
\hline $\mathrm{H}$ & -0.22600900 & 0.99289300 & -4.05202800 \\
\hline $\mathrm{H}$ & 0.61667100 & 3.27718100 & -3.89439100 \\
\hline $\mathrm{H}$ & 0.67358200 & -4.78378500 & 1.30735900 \\
\hline $\mathrm{H}$ & 4.51282400 & -0.96501400 & 0.53631800 \\
\hline $\mathrm{H}$ & 2.61500800 & 3.38109800 & -0.75795400 \\
\hline $\mathrm{Pd}$ & -0.97268800 & -0.34975100 & 0.15968200 \\
\hline C & -3.04052400 & -0.36202600 & 0.39827800 \\
\hline C & -3.83573500 & 0.01529500 & -0.69761700 \\
\hline C & -3.50888800 & -0.09168900 & 1.69921400 \\
\hline C & -5.02560900 & 0.71959400 & -0.49450200 \\
\hline $\mathrm{H}$ & -3.52717700 & -0.24206400 & -1.70647000 \\
\hline C & -4.69372700 & 0.61987600 & 1.89072400 \\
\hline $\mathrm{H}$ & -2.94281200 & -0.43420100 & 2.56169000 \\
\hline C & -5.45608900 & 1.03570000 & 0.79555800 \\
\hline $\mathrm{H}$ & -5.62011000 & 1.01588900 & -1.35560400 \\
\hline $\mathrm{H}$ & -5.02776500 & 0.83767200 & 2.90263300 \\
\hline $\mathrm{H}$ & -6.38588400 & 1.57682200 & 0.94749800 \\
\hline $\mathrm{O}$ & -3.75086200 & -3.39718000 & -0.90888000 \\
\hline
\end{tabular}

$\begin{array}{lrrr}\mathrm{N} & -2.74887700 & -2.68869000 & -0.97415100 \\ \mathrm{C} & -2.19273900 & -2.15745300 & 0.28341500 \\ \mathrm{O} & -2.18002600 & -2.37793000 & -2.02825400 \\ \mathrm{H} & -2.75876500 & -2.57895800 & 1.10662600 \\ \mathrm{H} & -1.14996900 & -2.52821600 & 0.31712500\end{array}$

XPhos Pd

Zero-point correction= 0.666567 (Hartree/Particle)

Thermal correction to Energy $=\quad 0.700053$

Thermal correction to Enthalpy $=\quad 0.700997$

Thermal correction to Gibbs Free Energy= 0.602026

Sum of electronic and zero-point Energies $=\quad-1636.559836$

Sum of electronic and thermal Energies $=\quad-1636.526349$

Sum of electronic and thermal Enthalpies $=\quad-1636.525405$

Sum of electronic and thermal Free Energies $=-1636.624376$

Electronic energy (M06): -1636.644736

C

C $\begin{array}{lll}1.10917300 & 0.26560300 & -0.19763900\end{array}$ $\begin{array}{llll}0.21621000 & -0.04373200 & 1.42933900\end{array}$ $\begin{array}{llll}0.91411400 & 0.11023100 & 2.64128200\end{array}$ $\begin{array}{lll}0.33679000 & -0.16838000 & 3.87981200\end{array}$ $0.91584000 \quad-0.03889200 \quad 4.79043100$ $\begin{array}{lll}-0.98191800 & -0.61175700 & 3.93307500\end{array}$ $\begin{array}{lll}-1.45008200 & -0.84656000 & 4.88537600\end{array}$ $\begin{array}{lll}-1.70852300 & -0.73407200 & 2.75041300\end{array}$ $\begin{array}{lll}-1.15346000 & -0.44369500 & 1.49153300\end{array}$ $\begin{array}{lll}-2.06745300 & -0.51076600 & 0.29282000\end{array}$ $\begin{array}{lll}-3.04521700 & 0.48037000 & 0.08194800\end{array}$ $\begin{array}{lll}-3.31866600 & 1.60815000 & 1.07924200\end{array}$ $\begin{array}{lll}-2.50612100 & 1.62152200 & 1.81174000\end{array}$ $\begin{array}{lll}-3.35916500 & 3.00213900 & 0.42230100\end{array}$ $\begin{array}{llll}-4.22708300 & 3.11990700 & -0.23652300\end{array}$ $\begin{array}{llll}-2.46057300 & 3.19108200 & -0.17238900\end{array}$ $\begin{array}{lll}-4.63084600 & 1.35351700 & 1.85089500\end{array}$ $\begin{array}{lll}-5.48766000 & 1.32039000 & 1.16709400\end{array}$ $\begin{array}{llll}-3.81083900 & 0.44754400 & -1.10759000\end{array}$ $\begin{array}{llll}-4.57085400 & 1.20538100 & -1.27184900\end{array}$ $\begin{array}{llll}-3.57771600 & -0.49919300 & -2.08797700\end{array}$ $\begin{array}{lll}-2.63794400 & -1.52658100 & -1.86871900\end{array}$ $\begin{array}{llll}-2.58348600 & -2.35030300 & -2.57329000\end{array}$ $\begin{array}{lll}-1.91644900 & -1.60035000 & -0.64860600\end{array}$ $\begin{array}{lll}-1.32498000 & -2.94152500 & -0.19210700\end{array}$ $\begin{array}{lll}-0.50116600 & -2.73062900 & 0.49761500\end{array}$ $\begin{array}{lll}-2.39408200 & -3.73332100 & 0.59353300\end{array}$ $\begin{array}{lll}-2.75167400 & -3.17407500 & 1.46380200\end{array}$ $\begin{array}{lll}-3.25899600 & -3.95387900 & -0.04378300\end{array}$ $\begin{array}{lll}-0.76949600 & -3.81176900 & -1.33103600\end{array}$ $-1.56701900-4.19125800-1.98105300$ $\begin{array}{lll}-0.06225200 & -3.25151100 & -1.95327200\end{array}$ $\begin{array}{lll}-4.81369400 & 2.15306100 & 2.57966000\end{array}$ $\begin{array}{lll}-3.42927900 & 3.77918400 & 1.19328100\end{array}$ $\begin{array}{lll}-0.25059700 & -4.68406900 & -0.91648400\end{array}$ $\begin{array}{llll}-1.98267000 & -4.68592600 & 0.94938500\end{array}$ $\begin{array}{lll}-4.14723700 & -0.48313300 & -3.01402000\end{array}$ $\begin{array}{lll}-2.74657900 & -1.05058900 & 2.79378300\end{array}$ $\begin{array}{llll}1.93621500 & 0.46960800 & 2.62361000\end{array}$ $-0.38157400-0.43638500-1.80228000$ $\begin{array}{lll}2.74966600 & -0.69561400 & -0.10418000\end{array}$ $\begin{array}{lll}3.87147900 & -0.24339500 & 0.85431700\end{array}$ $\begin{array}{llll}2.50277300 & -2.20948200 & 0.04976300\end{array}$ $3.12167800-0.53749500-1.12794700$ $\begin{array}{lll}5.16613100 & -1.03730500 & 0.59347900\end{array}$ $\begin{array}{lll}3.57078900 & -0.41687200 & 1.89457100\end{array}$ $\begin{array}{llll}4.07292000 & 0.82916500 & 0.75243900\end{array}$ $\begin{array}{llll}3.79147000 & -3.01939700 & -0.17679400\end{array}$ $\begin{array}{lll}2.11697600 & -2.41333600 & 1.05902300\end{array}$ $\begin{array}{lll}1.73230800 & -2.53273900 & -0.65776300\end{array}$ $\begin{array}{llll}4.93372600 & -2.54828800 & 0.73440900\end{array}$ $\begin{array}{lll}5.95030000 & -0.70442900 & 1.28600200\end{array}$ $\begin{array}{lll}-4.60304200 & 0.40144400 & 2.39169500\end{array}$ 


$\begin{array}{lrrr}\mathrm{H} & 5.52947000 & -0.81579100 & -0.42093100 \\ \mathrm{H} & 3.59150300 & -4.08683300 & -0.01653600 \\ \mathrm{H} & 4.09961600 & -2.91424800 & -1.22761000 \\ \mathrm{H} & 5.85552200 & -3.09946100 & 0.50810400 \\ \mathrm{H} & 4.68097700 & -2.77687300 & 1.78062200 \\ \mathrm{C} & 1.66250700 & 2.06037200 & -0.02096200 \\ \mathrm{C} & 2.52158600 & 2.48457800 & -1.23100000 \\ \mathrm{C} & 0.45198500 & 3.00128700 & 0.12760600 \\ \mathrm{H} & 2.27282300 & 2.14634000 & 0.89047500 \\ \mathrm{C} & 2.95268900 & 3.95895200 & -1.13778900 \\ \mathrm{H} & 1.93416000 & 2.33079000 & -2.14770700 \\ \mathrm{H} & 3.41034700 & 1.84798100 & -1.32094300 \\ \mathrm{C} & 0.88514100 & 4.47323400 & 0.22961300 \\ \mathrm{H} & -0.20439100 & 2.87182500 & -0.74467900 \\ \mathrm{H} & -0.13683900 & 2.72354300 & 1.00925300 \\ \mathrm{C} & 1.74618200 & 4.89352800 & -0.96948100 \\ \mathrm{H} & 3.52676500 & 4.23260300 & -2.03255100 \\ \mathrm{H} & 3.63191400 & 4.08509200 & -0.28125100 \\ \mathrm{H} & -0.00177700 & 5.11544200 & 0.30678000 \\ \mathrm{H} & 1.45793700 & 4.61951100 & 1.15769200 \\ \mathrm{H} & 2.08200600 & 5.93217800 & -0.85454300 \\ \mathrm{H} & 1.13366800 & 4.86085700 & -1.88266200\end{array}$

XPhos_preOA

Zero-point correction=

Thermal correction to Energy $=\quad 0.757556($ Hartree/Particle $)$

Thermal correction to Enthalpy= $\quad 0.800186$

Thermal correction to Gibbs Free Energy= $\quad 0.679505$

Sum of electronic and zero-point Energies $=\quad-1881.292038$

Sum of electronic and thermal Energies $=\quad-1881.250352$

Sum of electronic and thermal Enthalpies $=\quad-1881.249408$

Sum of electronic and thermal Free Energies $=-1881.370089$

Electronic energy (M06): -1881.33803

$\begin{array}{lrrr}\mathrm{P} & 0.97489900 & -0.92607700 & 0.31661300 \\ \mathrm{C} & 2.22745200 & 0.24824500 & 1.07082300 \\ \mathrm{C} & 3.32242400 & -0.28167500 & 1.78320500 \\ \mathrm{C} & 4.32933900 & 0.51994700 & 2.31660000 \\ \mathrm{H} & 5.15352300 & 0.06489800 & 2.85950600 \\ \mathrm{C} & 4.26623100 & 1.90059300 & 2.14466300 \\ \mathrm{H} & 5.04236400 & 2.54661200 & 2.54626700 \\ \mathrm{C} & 3.18439500 & 2.44960500 & 1.46214800 \\ \mathrm{C} & 2.15007800 & 1.65761000 & 0.92665900 \\ \mathrm{C} & 1.02708400 & 2.43536200 & 0.28503900 \\ \mathrm{C} & 1.12038200 & 2.83886800 & -1.06760900 \\ \mathrm{C} & 2.27086600 & 2.39564600 & -1.97003000 \\ \mathrm{H} & 2.81887400 & 1.60340900 & -1.44937600 \\ \mathrm{C} & 1.77996900 & 1.81102600 & -3.30804100 \\ \mathrm{H} & 1.31015000 & 2.57609800 & -3.93744100 \\ \mathrm{H} & 1.04656500 & 1.01376600 & -3.14849600 \\ \mathrm{C} & 3.26620800 & 3.54836700 & -2.21216700 \\ \mathrm{H} & 3.67967700 & 3.92175400 & -1.26906200 \\ \mathrm{H} & 2.77964300 & 4.38946400 & -2.72057400 \\ \mathrm{C} & 0.15261200 & 3.71176700 & -1.58225200 \\ \mathrm{H} & 0.22271600 & 4.03921700 & -2.61617200 \\ \mathrm{C} & -0.88534900 & 4.18614200 & -0.78549700 \\ \mathrm{C} & -0.97174800 & 3.78461700 & 0.54511000 \\ \mathrm{H} & -1.77829000 & 4.16600300 & 1.16534900 \\ \mathrm{C} & -0.02769900 & 2.91300600 & 1.10263300 \\ \mathrm{C} & -0.13071800 & 2.55953100 & 2.58642700 \\ \mathrm{H} & 0.57595600 & 1.74944700 & 2.78890600 \\ \mathrm{C} & 0.28168200 & 3.75724100 & 3.46648600 \\ \mathrm{H} & 1.30400400 & 4.08316700 & 3.24523600 \\ \mathrm{H} & -0.38491700 & 4.61327700 & 3.30576500 \\ \mathrm{C} & -1.52698100 & 2.04570800 & 2.98080900 \\ \mathrm{H} & -2.29371000 & 2.82373200 & 2.88582100 \\ \mathrm{H} & -1.82779500 & 1.20013400 & 2.35362800 \\ \mathrm{H} & 4.10114700 & 3.21325300 & -2.83973400 \\ \mathrm{H} & 2.62373300 & 1.39874700 & -3.87506200 \\ \mathrm{H} & -1.52447300 & 1.71737300 & 4.02753500 \\ \mathrm{H} & 0.23368700 & 3.48889300 & 4.52895800\end{array}$

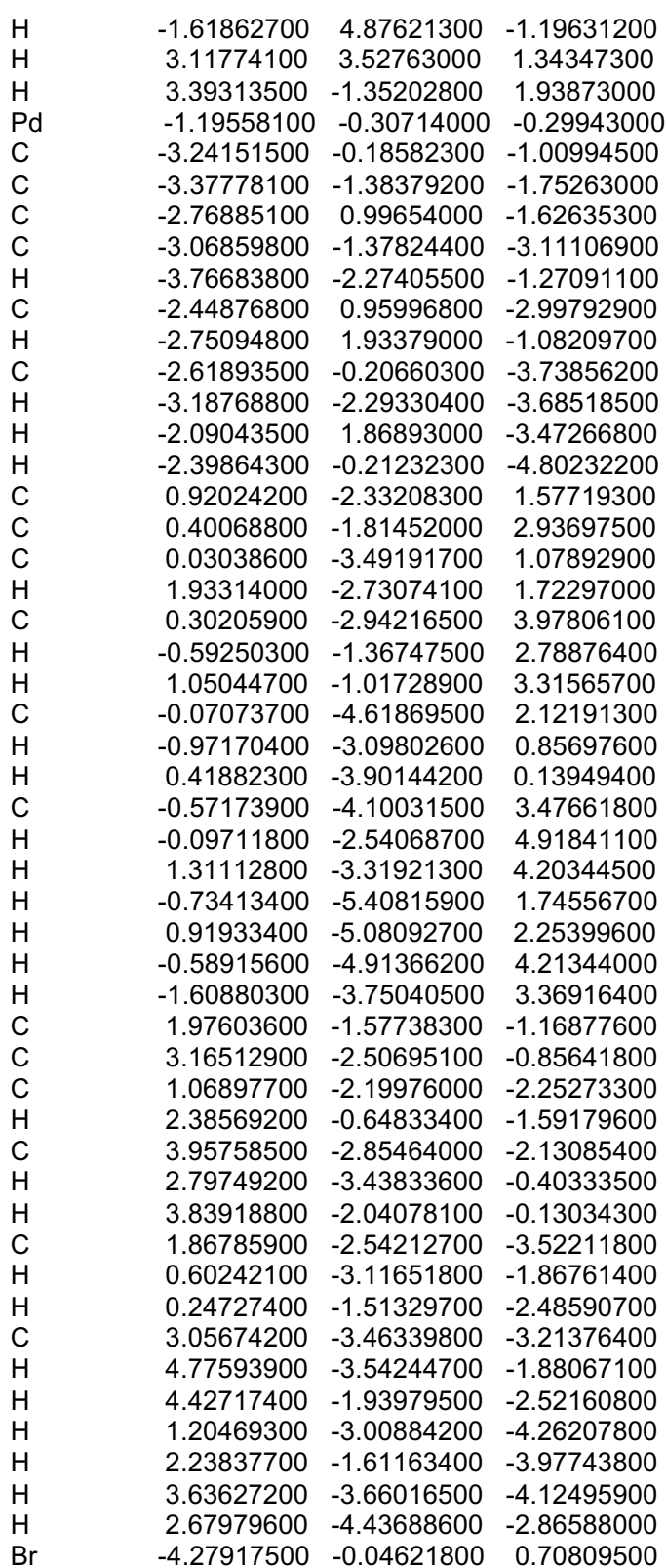

XPhos_OA_TS

Zero-point correction=

Thermal correction to Energy=

Thermal correction to Enthalpy=

0.757562 (Hartree/Particle)

Thermal correction to Gibbs Free Energy $=0.681188$

Sum of electronic and zero-point Energies $=\quad-1881.288025$

Sum of electronic and thermal Energies $=\quad-1881.247119$

Sum of electronic and thermal Enthalpies $=\quad-1881.246175$

Sum of electronic and thermal Free Energies $=-1881.364399$

Electronic energy (M06): -1881.329119

$\begin{array}{llrl}\mathrm{P} & -1.23828600 & -0.65695700 & 0.02251900 \\ \mathrm{C} & -2.30479600 & 0.72843500 & -0.63954900 \\ \mathrm{C} & -3.63962000 & 0.45424400 & -0.99956300 \\ \mathrm{C} & -4.51528700 & 1.44629400 & -1.43463700 \\ \mathrm{H} & -5.53625600 & 1.18861700 & -1.70326100 \\ \mathrm{C} & -4.06800400 & 2.76297900 & -1.51807400 \\ \mathrm{H} & -4.73452100 & 3.55565200 & -1.84730300 \\ \mathrm{C} & -2.74711700 & 3.05387100 & -1.18892400\end{array}$




\begin{tabular}{|c|c|c|c|}
\hline C & -1.84173300 & 2.06294200 & -0.76213300 \\
\hline C & -0.43423300 & 2.55069600 & -0.51975900 \\
\hline C & -0.07410600 & 3.09164000 & 0.73782400 \\
\hline C & -1.02151600 & 3.06431100 & 1.93604200 \\
\hline $\mathrm{H}$ & -1.87043200 & 2.42198600 & 1.67909500 \\
\hline C & -0.35935800 & 2.46982100 & 3.19353600 \\
\hline $\mathrm{H}$ & 0.44812600 & 3.10899800 & 3.56868100 \\
\hline $\mathrm{H}$ & 0.06829500 & 1.48267000 & 2.98783200 \\
\hline C & -1.59175800 & 4.46674700 & 2.22914700 \\
\hline $\mathrm{H}$ & -2.12587900 & 4.86788000 & 1.36083400 \\
\hline $\mathrm{H}$ & -0.79310400 & 5.17294400 & 2.48618200 \\
\hline C & 1.17303000 & 3.71458800 & 0.87522500 \\
\hline $\mathrm{H}$ & 1.45543300 & 4.13999800 & 1.83424300 \\
\hline C & 2.05032100 & 3.81259100 & -0.19990000 \\
\hline C & 1.69352600 & 3.27379100 & -1.43283600 \\
\hline $\mathrm{H}$ & 2.37890900 & 3.36129300 & -2.27139800 \\
\hline C & 0.45831000 & 2.63960500 & -1.61812800 \\
\hline C & 0.08976100 & 2.13171500 & -3.01251800 \\
\hline $\mathrm{H}$ & -0.81442600 & 1.52257800 & -2.92116800 \\
\hline C & -0.24268900 & 3.30801700 & -3.95395600 \\
\hline $\mathrm{H}$ & -1.06971000 & 3.91200400 & -3.56525700 \\
\hline $\mathrm{H}$ & 0.62432800 & 3.96793900 & -4.07906600 \\
\hline C & 1.17779700 & 1.23509600 & -3.63169000 \\
\hline $\mathrm{H}$ & 2.09904200 & 1.79225400 & -3.83927300 \\
\hline $\mathrm{H}$ & 1.43284300 & 0.39971400 & -2.97219600 \\
\hline $\mathrm{H}$ & -2.29294800 & 4.43081100 & 3.07197400 \\
\hline $\mathrm{H}$ & -1.09583500 & 2.36807800 & 4.00020800 \\
\hline $\mathrm{H}$ & 0.82580800 & 0.82484900 & -4.58606600 \\
\hline $\mathrm{H}$ & -0.52963100 & 2.93807400 & -4.94583300 \\
\hline $\mathrm{H}$ & 3.00760900 & 4.31420700 & -0.07971400 \\
\hline $\mathrm{H}$ & -2.38677100 & 4.07544900 & -1.27274300 \\
\hline $\mathrm{H}$ & -4.00924100 & -0.56358600 & -0.94970500 \\
\hline $\mathrm{Pd}$ & 1.14494300 & -0.56451100 & 0.15530100 \\
\hline C & 3.13405900 & -0.68959600 & 0.63795400 \\
\hline C & 3.24357600 & -1.51974300 & 1.76951500 \\
\hline C & 3.68553600 & 0.60253100 & 0.62147800 \\
\hline C & 3.83943600 & -1.00405400 & 2.92356000 \\
\hline $\mathrm{H}$ & 2.88590300 & -2.54290000 & 1.73259400 \\
\hline C & 4.27218900 & 1.09316700 & 1.79049300 \\
\hline $\mathrm{H}$ & 3.64133900 & 1.20489000 & -0.27822400 \\
\hline C & 4.35548300 & 0.29520400 & 2.93559400 \\
\hline $\mathrm{H}$ & 3.91542100 & -1.63019900 & 3.80912400 \\
\hline $\mathrm{H}$ & 4.67518800 & 2.10275300 & 1.79845900 \\
\hline $\mathrm{H}$ & 4.83405500 & 0.68023400 & 3.83175400 \\
\hline C & -1.79052200 & -2.13789500 & -1.01037700 \\
\hline C & -1.47128200 & -1.89756100 & -2.50229900 \\
\hline C & -1.11387300 & -3.43832800 & -0.52433700 \\
\hline $\mathrm{H}$ & -2.87619700 & -2.26797600 & -0.90386100 \\
\hline C & -1.86659600 & -3.10424100 & -3.37021400 \\
\hline $\mathrm{H}$ & -0.39349100 & -1.71181100 & -2.60769400 \\
\hline $\mathrm{H}$ & -1.98516900 & -0.99872100 & -2.86182600 \\
\hline C & -1.50882200 & -4.64352600 & -1.39604200 \\
\hline $\mathrm{H}$ & -0.02339200 & -3.30623800 & -0.55332500 \\
\hline $\mathrm{H}$ & -1.37902300 & -3.64314500 & 0.51914300 \\
\hline C & -1.20014200 & -4.39658500 & -2.87881600 \\
\hline $\mathrm{H}$ & -1.59693200 & -2.90853600 & -4.41593800 \\
\hline $\mathrm{H}$ & -2.96014000 & -3.22661200 & -3.34716100 \\
\hline $\mathrm{H}$ & -0.98485600 & -5.53982400 & -1.04019900 \\
\hline $\mathrm{H}$ & -2.58477700 & -4.84211400 & -1.27656200 \\
\hline $\mathrm{H}$ & -1.52970200 & -5.25071900 & -3.48425600 \\
\hline $\mathrm{H}$ & -0.11185700 & -4.31556600 & -3.01243900 \\
\hline C & -1.99537100 & -0.89596300 & 1.75403500 \\
\hline C & -3.39739000 & -1.53298300 & 1.82668800 \\
\hline C & -1.02131800 & -1.61981900 & 2.70988800 \\
\hline $\mathrm{H}$ & -2.08352300 & 0.14074700 & 2.11204800 \\
\hline C & -3.92874200 & -1.55946200 & 3.27245200 \\
\hline $\mathrm{H}$ & -3.35658200 & -2.56229700 & 1.44371000 \\
\hline $\mathrm{H}$ & -4.10369500 & -0.98427500 & 1.19541000 \\
\hline C & -1.55760600 & -1.64011800 & 4.15112100 \\
\hline $\mathrm{H}$ & -0.86661800 & -2.65307100 & 2.37147700 \\
\hline
\end{tabular}

$\begin{array}{lrrr}\mathrm{H} & -0.03805200 & -1.13547300 & 2.67463300 \\ \mathrm{C} & -2.95714500 & -2.26711600 & 4.22665100 \\ \mathrm{H} & -4.91201500 & -2.04713100 & 3.29461800 \\ \mathrm{H} & -4.08496600 & -0.52590100 & 3.61507400 \\ \mathrm{H} & -0.85853500 & -2.18541400 & 4.79823800 \\ \mathrm{H} & -1.59997700 & -0.61006200 & 4.53491500 \\ \mathrm{H} & -3.33872200 & -2.22915500 & 5.25503100 \\ \mathrm{H} & -2.89298400 & -3.33161600 & 3.95590500 \\ \mathrm{Br} & 3.15115900 & -1.78880500 & -1.33094500\end{array}$

XPhos OA prod

Zero-point correction= Thermal correction to Energy=

Thermal correction to Enthalpy= 0.758924 (Hartree/Particle) 0.800161 0.801105

Thermal correction to Gibbs Free Energy= $\quad 0.683864$ Sum of electronic and zero-point Energies $=\quad-1881.306510$ Sum of electronic and thermal Energies $=\quad-1881.265273$ Sum of electronic and thermal Enthalpies $=\quad-1881.264329$ Sum of electronic and thermal Free Energies $=-1881.381570$ Electronic energy (M06): -1881.348597

C

$\mathrm{C}$

$\mathrm{H}$

C

$\mathrm{H}$

$\mathrm{H}$

$\mathrm{H}$

$\mathrm{H}$

$\mathrm{H}$

$\mathrm{H}$

$\mathrm{H}$

$\mathrm{Pd}$

$\mathrm{Pd} \quad 0.76372200 \quad-0.78539600 \quad 0.06204500$

C $\quad 2.61481800 \quad-1.54966900 \quad 0.35487900$

C $\quad 3.11999200 \quad-1.55807300 \quad 1.65867000$

$\begin{array}{llll}C & 3.41444000 & -2.00865400 & -0.69597800\end{array}$

C $\quad 4.44217900 \quad-1.95101100 \quad 1.89900000$

$\mathrm{H} \quad 2.49340700 \quad-1.26355200 \quad 2.49625500$

$\begin{array}{llll}\text { C } & 4.73527600 & -2.40095800 & -0.45320600\end{array}$

$\mathrm{H} \quad 3.01829800 \quad-2.06769400 \quad-1.70498100$

$\begin{array}{llll}\text { C } & 5.25454100 & -2.36781100 & 0.84311000\end{array}$ 


\begin{tabular}{lrrr}
$\mathrm{H}$ & 4.82794900 & -1.94518200 & 2.91638600 \\
$\mathrm{H}$ & 5.35269300 & -2.74747500 & -1.27945900 \\
$\mathrm{H}$ & 6.27748200 & -2.68423800 & 1.03149800 \\
$\mathrm{C}$ & -2.82667500 & -0.49284400 & -1.23733700 \\
$\mathrm{C}$ & -2.27126300 & -0.48283800 & -2.67896900 \\
$\mathrm{C}$ & -3.33712200 & -1.90266500 & -0.86744300 \\
$\mathrm{H}$ & -3.68359400 & 0.19518500 & -1.19465800 \\
$\mathrm{C}$ & -3.31302300 & -0.98772400 & -3.69140700 \\
$\mathrm{H}$ & -1.38250600 & -1.12699300 & -2.71924900 \\
$\mathrm{H}$ & -1.94628200 & 0.52746700 & -2.95611500 \\
$\mathrm{C}$ & -4.38106400 & -2.39716700 & -1.88443000 \\
$\mathrm{H}$ & -2.49203400 & -2.59948500 & -0.83286200 \\
$\mathrm{H}$ & -3.78138100 & -1.90015900 & 0.13379700 \\
$\mathrm{C}$ & -3.83228900 & -2.38275200 & -3.31754200 \\
$\mathrm{H}$ & -2.87261300 & -0.99819800 & -4.69665200 \\
$\mathrm{H}$ & -4.15672700 & -0.28192800 & -3.72703900 \\
$\mathrm{H}$ & -4.70404100 & -3.40919700 & -1.60992700 \\
$\mathrm{H}$ & -5.27619700 & -1.75819900 & -1.83249400 \\
$\mathrm{H}$ & -4.60627200 & -2.70382800 & -4.02638600 \\
$\mathrm{H}$ & -3.01013400 & -3.10823300 & -3.39680300 \\
$\mathrm{C}$ & -2.31710800 & 0.28834800 & 1.66435500 \\
$\mathrm{C}$ & -3.79126800 & 0.74034400 & 1.71707800 \\
$\mathrm{C}$ & -2.10109500 & -1.00003800 & 2.49017100 \\
$\mathrm{H}$ & -1.71684200 & 1.07907600 & 2.13939300 \\
$\mathrm{C}$ & -4.27126900 & 0.90636600 & 3.17128800 \\
$\mathrm{H}$ & -4.42668200 & -0.00196200 & 1.21576600 \\
$\mathrm{H}$ & -3.92432500 & 1.68781600 & 1.18372700 \\
$\mathrm{C}$ & -2.58807100 & -0.82067200 & 3.93730500 \\
$\mathrm{H}$ & -2.63812300 & -1.83722200 & 2.02793900 \\
$\mathrm{H}$ & -1.04361300 & -1.28266900 & 2.47556100 \\
$\mathrm{C}$ & -4.05486000 & -0.37155100 & 3.99370400 \\
$\mathrm{H}$ & -5.33082800 & 1.19330700 & 3.17735600 \\
$\mathrm{H}$ & -3.72058800 & 1.73642200 & 3.63829700 \\
$\mathrm{H}$ & -2.45334000 & -1.76010200 & 4.48775900 \\
$\mathrm{H}$ & -1.95996600 & -0.07033100 & 4.44087300 \\
$\mathrm{H}$ & -4.36751300 & -0.21096100 & 5.03334600 \\
$\mathrm{H}$ & -4.69451200 & -1.17250100 & 3.59427000 \\
$\mathrm{Br}$ & 0.10570200 & -3.24063200 & 0.15433600 \\
& & & \\
\hline
\end{tabular}

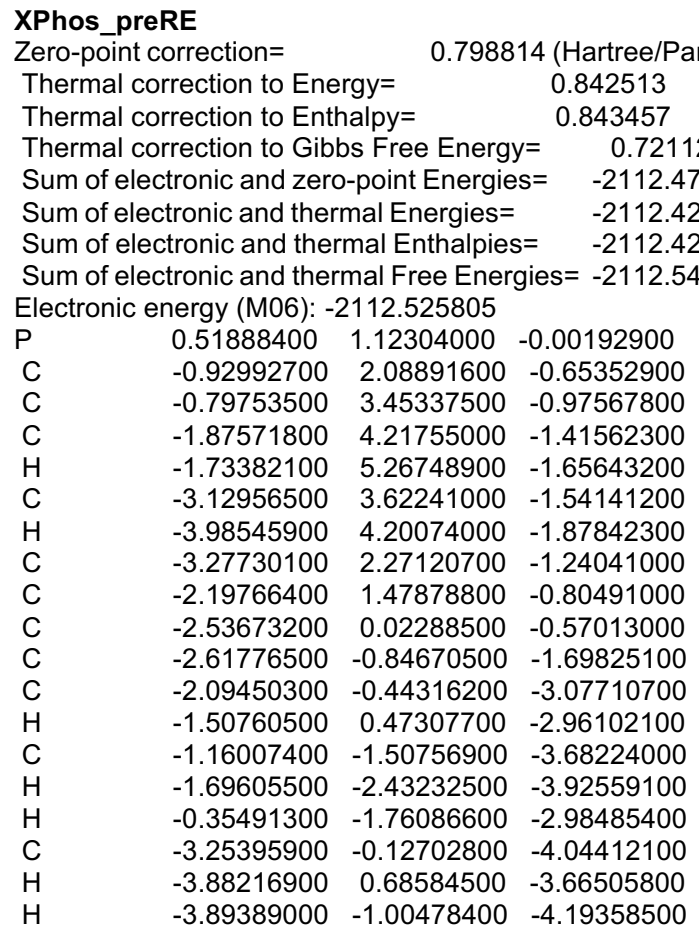

\begin{tabular}{|c|c|c|c|}
\hline C & -3.26303400 & -2.07972800 & -1.55359900 \\
\hline $\mathrm{H}$ & -3.35098700 & -2.73932700 & -2.41225100 \\
\hline C & -3.80104000 & -2.47492400 & -0.33072200 \\
\hline C & -3.68893400 & -1.64278600 & 0.77781700 \\
\hline $\mathrm{H}$ & -4.09565200 & -1.96622800 & 1.73010100 \\
\hline C & -3.08168000 & -0.38295800 & 0.68057300 \\
\hline C & -3.13137000 & 0.55400700 & 1.88706200 \\
\hline $\mathrm{H}$ & -2.49130200 & 1.41559200 & 1.67031700 \\
\hline C & -4.55971600 & 1.10160800 & 2.09182800 \\
\hline $\mathrm{H}$ & -4.91673500 & 1.63553500 & 1.20455400 \\
\hline $\mathrm{H}$ & -5.26523400 & 0.28955900 & 2.30341000 \\
\hline C & -2.61295100 & -0.10107000 & 3.18040300 \\
\hline $\mathrm{H}$ & -3.27903200 & -0.89786700 & 3.52846400 \\
\hline $\mathrm{H}$ & -1.62613900 & -0.55138100 & 4253300 \\
\hline $\mathrm{H}$ & -2.86421900 & 0.17518400 & -5.02356200 \\
\hline $\mathrm{H}$ & -0.71312700 & -1.13381800 & -4.61120000 \\
\hline $\mathrm{H}$ & -2.55480600 & 0.64806000 & 3.97961000 \\
\hline $\mathrm{H}$ & -4.58419300 & 1.79757400 & 2.93899800 \\
\hline $\mathrm{H}$ & -4.30200700 & -3.43523100 & -0.24056300 \\
\hline $\mathrm{H}$ & -4.25057700 & 1.80161800 & -1.35098100 \\
\hline $\mathrm{H}$ & 0.16986800 & 3.93546600 & -0.88990800 \\
\hline $\mathrm{Pd}$ & 0.05576000 & -1.26508900 & 0.01196600 \\
\hline C & 1.94557300 & -1.92988700 & -0.19856200 \\
\hline C & 2.77775800 & -2.15867200 & 0.90232700 \\
\hline C & 2.44203300 & -2.16003400 & -1.48890100 \\
\hline C & 4.10473300 & -2.56055500 & 0.71082600 \\
\hline $\mathrm{H}$ & 2.40259100 & -2.04370900 & 1.91350000 \\
\hline C & 3.76983800 & -2.56476800 & -1.67518800 \\
\hline $\mathrm{H}$ & 1.80531900 & -2.02726600 & -2.35886100 \\
\hline C & 4.60878200 & -2.75712200 & -0.57600400 \\
\hline $\mathrm{H}$ & 4.73954300 & -2.73043200 & 1.57749700 \\
\hline $\mathrm{H}$ & 4.14011700 & -2.73661700 & -2.68369000 \\
\hline $\mathrm{H}$ & 5.63874400 & -3.07289400 & -0.72021900 \\
\hline $\mathrm{O}$ & 0.71658200 & -4.19003700 & 2.14246500 \\
\hline $\mathrm{N}$ & -0.26853100 & -3.56261800 & 1.74268600 \\
\hline C & -0.39944900 & -3.29477400 & 0.30907800 \\
\hline 0 & -1.14324300 & -3.11092400 & 2.50122000 \\
\hline $\mathrm{H}$ & 0.22059300 & -4.00646400 & -0.22887300 \\
\hline $\mathrm{H}$ & -1.45738100 & -3.35480300 & 0.05224100 \\
\hline C & 0.64949700 & 1.76525800 & 1.78011500 \\
\hline C & 1.43324400 & 0.79805400 & 2.69536800 \\
\hline C & 1.14214100 & 3.21723800 & 1.94691400 \\
\hline $\mathrm{H}$ & -0.39856700 & 1.73510600 & 2.11025800 \\
\hline C & 1.38878400 & 1.25710800 & 4.16298300 \\
\hline $\mathrm{H}$ & 2.47856900 & 0.73210000 & 2.36784000 \\
\hline $\mathrm{H}$ & 1.01719200 & -0.21145900 & 2.60686500 \\
\hline C & 1.09400200 & 3.65689100 & 3.42248400 \\
\hline $\mathrm{H}$ & 2.17583800 & 3.30547800 & 1.58636500 \\
\hline $\mathrm{H}$ & 0.53341300 & 3.90177600 & 1.34607200 \\
\hline C & 1.88342500 & 2.70048500 & 4.32636100 \\
\hline $\mathrm{H}$ & 1.98720600 & 0.57346500 & 4.77787400 \\
\hline $\mathrm{H}$ & 0.35527400 & 1.18286100 & 4.53147000 \\
\hline $\mathrm{H}$ & 1.48105300 & 4.68010300 & 3.51243400 \\
\hline $\mathrm{H}$ & 0.04579700 & 3.68877300 & 3.75426000 \\
\hline $\mathrm{H}$ & 1.80298900 & 3.01554500 & 5.37436700 \\
\hline $\mathrm{H}$ & 2.95099200 & 2.75213700 & 4.06491400 \\
\hline C & 1.97446500 & 1.83686100 & -0.95803200 \\
\hline C & 3.34566100 & 1.38251400 & -0.40920100 \\
\hline C & 1.84379600 & 1.49420500 & -2.45916300 \\
\hline $\mathrm{H}$ & 1.92898500 & 2.92972300 & -0.85329800 \\
\hline C & 4.49770000 & 1.97997300 & -1.23661700 \\
\hline $\mathrm{H}$ & 3.41111100 & 0.29055100 & -0.42921300 \\
\hline $\mathrm{H}$ & 3.45855400 & 1.68601700 & 0.63699100 \\
\hline C & 3.00852800 & 2.07869200 & -3.27661800 \\
\hline $\mathrm{H}$ & 1.83244400 & 0.40335500 & -2.57420400 \\
\hline $\mathrm{H}$ & 0.89053500 & 1.86704400 & -2.85276800 \\
\hline C & 4.36842100 & 1.62865500 & -2.72459500 \\
\hline $\mathrm{H}$ & 5.45412100 & 1.61720100 & -0.83997400 \\
\hline $\mathrm{H}$ & 4.50441800 & 3.07456800 & -1.12009000 \\
\hline $\mathrm{H}$ & 2.90267600 & 1.78185400 & -4.32794700 \\
\hline
\end{tabular}


$\begin{array}{llll}\mathrm{H} & 2.95253600 & 3.17753900 & -3.25550900 \\ \mathrm{H} & 5.18317200 & 2.08860400 & -3.29821300 \\ \mathrm{H} & 4.46822800 & 0.54080600 & -2.84563800\end{array}$

XPhos_RE_TS

Zero-point correction=

Thermal correction to Energy=

Thermal correction to Enthalpy=

0.797252 (Hartree/Particle)

Thermal correction to Gibbs Free Energy=
Sum of electronic and zero-point Energies=

0.840702

0.841646 0.718529

Sum of electronic and thermal Energies=

$-2112.447752$

$-2112.404303$

Sum of electronic and thermal Enthalpies $=\quad-2112.403358$

Sum of electronic and thermal Free Energies -2112.526475

Electronic energy (M06): --2112.508583

$\begin{array}{llll}\mathrm{P} & -0.02034600 & 1.27764100 & -0.05528200\end{array}$

C $\quad-1.74824900 \quad 1.65050900-0.64739200$

C $\quad-2.09488000 \quad 2.95804600 \quad-1.03898800$

C $\quad-3.38819300 \quad 3.29865100 \quad-1.42934000$

H $\quad-3.61468300 \quad 4.31958000 \quad-1.72467900$

C $\quad-4.38066800 \quad 2.32064000 \quad-1.43426600$

H $\quad-5.39777500 \quad 2.56547800 \quad-1.72817600$

C $\quad-4.05505300 \quad 1.01625400 \quad-1.07133600$

C $\quad-2.75147300 \quad 0.64918100-0.68724200$

C $\quad-2.55744100 \quad-0.82021300 \quad-0.39264900$

C $\quad-2.32944900-1.71241600-1.47516700$

C $\quad-2.11264600-1.21684200 \quad-2.90532400$

$\mathrm{H} \quad-1.94763600 \quad-0.13628000 \quad-2.86410100$

C $\quad-0.86771100-1.83607000 \quad-3.56679200$

$\mathrm{H} \quad-0.97486700 \quad-2.91702500-3.71539800$

$\mathrm{H} \quad 0.02369900-1.66344500-2.95447100$

C $\quad-3.36613200-1.45909200-3.77080500$

$\mathrm{H} \quad-4.24397000-0.95518500-3.35245000$

$\mathrm{H} \quad-3.59635300-2.52892800-3.84213300$

C $\quad-2.35834000 \quad-3.09099500 \quad-1.23144500$

H $\quad-2.19885400 \quad-3.78247900-2.05412200$

$\begin{array}{llll}\text { C } & -2.60141000 & -3.59393000 & 0.04486700\end{array}$

$\begin{array}{llll}\text { C } & -2.81987500 & -2.71544900 & 1.10241600\end{array}$

$\begin{array}{llll}\mathrm{H} & -3.01317400 & -3.11382400 & 2.09405400\end{array}$

C $\quad-2.80824000 \quad-1.32759800 \quad 0.90758200$

$\begin{array}{llll}\text { C } & -3.11772500 & -0.41229700 & 2.09056900\end{array}$

$\begin{array}{llll}\mathrm{H} & -2.87943700 & 0.61328600 & 1.79026600\end{array}$

$\begin{array}{llll}\text { C } & -4.62042900 & -0.44356400 & 2.43693800\end{array}$

$\mathrm{H} \quad-5.23448400 \quad-0.13478500 \quad 1.58393700$

$\mathrm{H} \quad-4.93524900 \quad-1.45233400 \quad 2.72982600$

C $\quad-2.26528500 \quad-0.74248500 \quad 3.33070600$

$\mathrm{H} \quad-2.54053300 \quad-1.70949100 \quad 3.76698300$

H $\quad-1.19882400 \quad-0.78430300 \quad 3.08642500$

$\mathrm{H} \quad-3.21077300-1.07992200-4.78808900$

$\mathrm{H} \quad-0.70112200 \quad-1.38588700 \quad-4.55280700$

$\mathrm{H} \quad \begin{array}{llll}\mathrm{H} & -2.41794300 & 0.01752400 & 4.10666300\end{array}$

$\mathrm{H} \quad-4.83675000 \quad 0.23231100 \quad 3.27313700$

$\mathrm{H} \quad-2.63147600 \quad-4.66762600 \quad 0.21233300$

$\begin{array}{llll}\mathrm{H} & -4.82153600 & 0.24657600 & -1.09330700\end{array}$

$\mathrm{H} \quad-1.33646400 \quad 3.73313600 \quad-1.04960400$

$\begin{array}{llll}\mathrm{Pd} & 0.55634400 & -1.02517200 & 0.10287200\end{array}$

C $\quad 2.51659600-1.69122200-0.07670700$

$\begin{array}{llll}\text { C } & 3.42699300 & -1.30185300 & 0.92025100\end{array}$

C $\quad 2.98611400-1.87538900-1.39155100$

C $\quad 4.75718100-1.03036000 \quad 0.58911200$

$\mathrm{H} \quad 3.09649200-1.20568000 \quad 1.94981300$

C $\quad 4.31426000 \quad-1.59158200-1.71345900$

$\begin{array}{llll}\mathrm{H} & 2.31269500 & -2.23780000 & -2.16345600\end{array}$

C $\quad 5.20669500 \quad-1.16303600 \quad-0.72647900$

$\begin{array}{llll}\mathrm{H} & 5.44478100 & -0.71637900 & 1.37095100\end{array}$

$\mathrm{H} \quad 4.65470700-1.72255400 \quad-2.73814000$

$\mathrm{H} \quad 6.24399200 \quad-0.95760900 \quad-0.97605400$

$\begin{array}{llll}\mathrm{O} & 2.36910400 & -4.39760800 & 1.94370100\end{array}$

N $\quad 1.61270400 \quad-3.43868000 \quad 1.80593300$

$\begin{array}{llll}\text { C } & 1.18577900 & -3.08243200 & 0.44070100\end{array}$ $\begin{array}{rrr}1.19272600 & -2.73710100 & 2.73354500 \\ 1.56614700 & -3.83361400 & -0.24253400 \\ 0.07731100 & -3.11941100 & 0.46280200 \\ -0.00274000 & 2.13555400 & 1.64107200 \\ 1.09594600 & 1.56131000 & 2.56405700 \\ 0.03007500 & 3.67682500 & 1.63907400 \\ -0.96941000 & 1.82709500 & 2.06633200 \\ 0.98618400 & 2.13440800 & 3.98735300 \\ 2.08776000 & 1.79723900 & 2.15649400 \\ 1.02482000 & 0.46829700 & 2.58892600 \\ -0.07363400 & 4.23998400 & 3.06924600 \\ 0.96692600 & 4.02791200 & 1.18524300 \\ -0.78920000 & 4.07956600 & 1.03448100 \\ 1.01510200 & 3.66925100 & 3.98794400 \\ 1.79782100 & 1.73486400 & 4.60834400 \\ 0.04651100 & 1.78861300 & 4.44277400 \\ -0.01576300 & 5.33580200 & 3.03824900 \\ -1.06287000 & 3.99065300 & 3.48062500 \\ 0.89113000 & 4.05423100 & 5.00815200 \\ 2.00033900 & 4.01445900 & 3.64066100 \\ 1.06026200 & 2.30643500 & -1.20491700 \\ 2.54177700 & 2.27735500 & -0.76862100 \\ 0.92044000 & 1.80050000 & -2.65739700 \\ 0.71830900 & 3.35031800 & -1.16684700 \\ 3.43191100 & 3.06955200 & -1.74246900 \\ 2.88682700 & 1.23738300 & -0.72341600 \\ 2.65365100 & 2.68949500 & 0.24029700 \\ 1.81595200 & 2.59123600 & -3.62605200 \\ 1.20304900 & 0.73930200 & -2.68858300 \\ -0.12460700 & 1.86088400 & -2.98363400 \\ 3.28546400 & 2.56349600 & -3.18337900 \\ 4.47762000 & 2.99978100 & -1.41749900 \\ 3.16132600 & 4.13568700 & -1.70190600 \\ 1.71201700 & 2.18142900 & -4.63905600 \\ 1.46932300 & 3.63455000 & -3.67456200 \\ 3.90129100 & 3.16463000 & -3.86459200 \\ 3.66204600 & 1.53203900 & -3.24117700\end{array}$

FOR DISTORTION-INTERACTION ANALYSIS

XPhos_Pd

Electronic energy (M06): -1636.644736

XPhos_Pd_@preOA_TS geometry

Electronic energy (M06): -1636.64075

$\begin{array}{llrl}\text { P } & -0.97489900 & -0.92607700 & -0.31661300 \\ \mathrm{C} & -2.22745200 & 0.24824500 & -1.07082300 \\ \mathrm{C} & -3.32242400 & -0.28167600 & -1.78320500 \\ \mathrm{C} & -4.32933900 & 0.51994600 & -2.31660000 \\ \mathrm{H} & -5.15352300 & 0.06489700 & -2.85950600 \\ \mathrm{C} & -4.26623100 & 1.90059200 & -2.14466300 \\ \mathrm{H} & -5.04236400 & 2.54661100 & -2.54626700 \\ \mathrm{C} & -3.18439500 & 2.44960400 & -1.46214800 \\ \mathrm{C} & -2.15007800 & 1.65761000 & -0.92665900 \\ \mathrm{C} & -1.02708400 & 2.43536200 & -0.28503900 \\ \mathrm{C} & -1.12038300 & 2.83886800 & 1.06760900 \\ \mathrm{C} & -2.27086600 & 2.39564600 & 1.97003000 \\ \mathrm{H} & -2.81887400 & 1.60340900 & 1.44937600 \\ \mathrm{C} & -1.77996900 & 1.81102600 & 3.30804100 \\ \mathrm{H} & -1.31015000 & 2.57609800 & 3.93744100 \\ \mathrm{H} & -1.04656500 & 1.01376600 & 3.14849600 \\ \mathrm{C} & -3.26620900 & 3.54836600 & 2.21216700 \\ \mathrm{H} & -3.67967800 & 3.92175300 & 1.26906200 \\ \mathrm{H} & -2.77964400 & 4.38946400 & 2.72057400 \\ \mathrm{C} & -0.15261300 & 3.71176700 & 1.58225200 \\ \mathrm{H} & -0.22271700 & 4.03921700 & 2.61617200 \\ \mathrm{C} & 0.88534800 & 4.18614200 & 0.78549700 \\ \mathrm{C} & 0.97174700 & 3.78461700 & -0.54511000 \\ \mathrm{H} & 1.77828900 & 4.16600300 & -1.16534900 \\ \mathrm{C} & 0.02769800 & 2.91300600 & -1.10263300 \\ \mathrm{C} & 0.13071800 & 2.55953100 & -2.58642700\end{array}$




\begin{tabular}{lrrr}
$\mathrm{H}$ & -0.57595600 & 1.74944700 & -2.78890600 \\
$\mathrm{C}$ & -0.28168300 & 3.75724100 & -3.46648600 \\
$\mathrm{H}$ & -1.30400500 & 4.08316700 & -3.24523600 \\
$\mathrm{H}$ & 0.38491600 & 4.61327700 & -3.30576500 \\
$\mathrm{C}$ & 1.52698100 & 2.04570800 & -2.98080900 \\
$\mathrm{H}$ & 2.29370900 & 2.82373200 & -2.88582100 \\
$\mathrm{H}$ & 1.82779500 & 1.20013400 & -2.35362800 \\
$\mathrm{H}$ & -4.10114800 & 3.21325200 & 2.83973400 \\
$\mathrm{H}$ & -2.62373300 & 1.39874700 & 3.87506200 \\
$\mathrm{H}$ & 1.52447300 & 1.71737300 & -4.02753500 \\
$\mathrm{H}$ & -0.23368800 & 3.48889300 & -4.52895800 \\
$\mathrm{H}$ & 1.61862600 & 4.87621300 & 1.19631200 \\
$\mathrm{H}$ & -3.11774200 & 3.52762900 & -1.34347300 \\
$\mathrm{H}$ & -3.39313500 & -1.35202900 & -1.93873000 \\
$\mathrm{Pd}$ & 1.19558100 & -0.30714000 & 0.29943000 \\
$\mathrm{C}$ & -0.92024200 & -2.33208300 & -1.57719300 \\
$\mathrm{C}$ & -0.40068800 & -1.81452000 & -2.93697500 \\
$\mathrm{C}$ & -0.03038500 & -3.49191700 & -1.07892900 \\
$\mathrm{H}$ & -1.93314000 & -2.73074100 & -1.72297000 \\
$\mathrm{C}$ & -0.30205900 & -2.94216500 & -3.97806100 \\
$\mathrm{H}$ & 0.59250300 & -1.36747500 & -2.78876400 \\
$\mathrm{H}$ & -1.05044700 & -1.01728900 & -3.31565700 \\
$\mathrm{C}$ & 0.07073800 & -4.61869500 & -2.12191300 \\
$\mathrm{H}$ & 0.97170500 & -3.09802600 & -0.85697600 \\
$\mathrm{H}$ & -0.41882200 & -3.90144200 & -0.13949400 \\
$\mathrm{C}$ & 0.57174000 & -4.10031500 & -3.47661800 \\
$\mathrm{H}$ & 0.09711800 & -2.54068700 & -4.91841100 \\
$\mathrm{H}$ & -1.31112700 & -3.31921300 & -4.20344500 \\
$\mathrm{H}$ & 0.73413500 & -5.40815900 & -1.74556700 \\
$\mathrm{H}$ & -0.91933300 & -5.08092700 & -2.25399600 \\
$\mathrm{H}$ & 0.58915700 & -4.91366200 & -4.21344000 \\
$\mathrm{H}$ & 1.60880400 & -3.75040500 & -3.36916400 \\
$\mathrm{C}$ & -1.97603600 & -1.57738300 & 1.16877600 \\
$\mathrm{C}$ & -3.16512900 & -2.50695200 & 0.85641800 \\
$\mathrm{C}$ & -1.06897700 & -2.19976000 & 2.25273300 \\
$\mathrm{H}$ & -2.38569200 & -0.64833400 & 1.59179600 \\
$\mathrm{C}$ & -3.95758500 & -2.85464100 & 2.13085400 \\
$\mathrm{H}$ & -2.79749100 & -3.43833600 & 0.40333500 \\
$\mathrm{H}$ & -3.83918800 & -2.04078200 & 0.13034300 \\
$\mathrm{C}$ & -1.86785900 & -2.54212700 & 3.52211800 \\
$\mathrm{H}$ & -0.60242000 & -3.11651800 & 1.86761400 \\
$\mathrm{H}$ & -0.24727400 & -1.51329700 & 2.48590700 \\
$\mathrm{H}$ & -3.05674100 & -3.46339900 & 3.21376400 \\
$\mathrm{H}$ & -4.77593800 & -3.54244800 & 1.88067100 \\
$\mathrm{H}$ & -4.42717400 & -1.93979600 & 2.52160800 \\
& -1.20469200 & -3.00884200 & 4.26207800 \\
-2.23837700 & -1.61163400 & 3.97743800 \\
-3.63627100 & -3.66016600 & 4.12495900 \\
\hline & -2.67979500 & -4.43688600 & 2.86588000
\end{tabular}

XPhos distortion for complexation: $2.50 \mathrm{kcal} / \mathrm{mol}$

\section{tBuXPhos_Pd}

Electronic energy (M06): -1481.868123

XPhos_Pd_@preOA_TS geometry

Electronic energy (M06): -1481.85783

$\begin{array}{lrrr}\text { P } & 1.55309300 & -0.03608400 & -0.05000100 \\ \mathrm{C} & 0.45395500 & -0.03936400 & 1.47547600 \\ \mathrm{C} & 1.06345300 & -0.04718800 & 2.74787100 \\ \mathrm{C} & 0.34174200 & -0.01317200 & 3.93792700 \\ \mathrm{H} & 0.86523200 & -0.01952800 & 4.89026500 \\ \mathrm{C} & -1.04869500 & 0.03154300 & 3.88682300 \\ \mathrm{H} & -1.63980100 & 0.06085100 & 4.79828100 \\ \mathrm{C} & -1.67773300 & 0.04275200 & 2.64629500 \\ \mathrm{C} & -0.96844300 & 0.00928700 & 1.42743500 \\ \mathrm{C} & -1.86849800 & 0.04441300 & 0.20917600 \\ \mathrm{C} & -2.32555700 & 1.29297900 & -0.28575600 \\ \mathrm{C} & -1.84172800 & 2.62550000 & 0.28379300 \\ \mathrm{H} & -0.94786300 & 2.42978400 & 0.88137800 \\ \mathrm{C} & -1.45268700 & 3.63912400 & -0.80875700\end{array}$

\begin{abstract}
$\begin{array}{lll}-2.32701300 & 3.98430400 & -1.37381200\end{array}$
$\begin{array}{lll}-0.74410500 & 3.20518200 & -1.52164600\end{array}$

$\begin{array}{lll}-2.89771600 & 3.23728400 & 1.22826000\end{array}$

$\begin{array}{lll}-3.12777400 & 2.56531300 & 2.06172900\end{array}$

$\begin{array}{lll}-3.83314300 & 3.43951600 & 0.69265700\end{array}$

$\begin{array}{lll}-3.31385400 & 1.31088300 & -1.27822400\end{array}$

$\begin{array}{lll}-3.67444600 & 2.26495500 & -1.65262400\end{array}$

$\begin{array}{lll}-3.85773400 & 0.13244200 & -1.77834100\end{array}$

$\begin{array}{lll}-3.42494000 & -1.08906000 & -1.27382200\end{array}$

$\begin{array}{lll}-3.86747300 & -2.00565900 & -1.65129600\end{array}$

$-2.44798700 \quad-1.15863700 \quad-0.27247500$

$\begin{array}{lll}-2.11572200 & -2.52477200 & 0.32731100\end{array}$

$\begin{array}{lll}-1.18623800 & -2.42348500 & 0.89545700\end{array}$

$\begin{array}{lll}-3.21041100 & -2.96815400 & 1.32133400\end{array}$

$\begin{array}{lll}-3.32657700 & -2.25126300 & 2.14030900\end{array}$

$\begin{array}{lll}-4.17961500 & -3.06291200 & 0.81691000\end{array}$

$\begin{array}{lll}-1.89708600 & -3.61846500 & -0.73458000\end{array}$

$-2.83363700 \quad-3.89130700 \quad-1.23472600$

$\begin{array}{lll}-1.18957400 & -3.29830100 & -1.50418600\end{array}$

$\begin{array}{lll}-2.53742200 & 4.18502600 & 1.64720200\end{array}$

$\begin{array}{llll}-0.99217900 & 4.52523400 & -0.35507200\end{array}$

$\begin{array}{lll}-1.50848300 & -4.52834200 & -0.26156000\end{array}$

$\begin{array}{lll}-2.96094600 & -3.94312800 & 1.75807900\end{array}$

$\begin{array}{lll}-4.62824600 & 0.16516700 & -2.54476400\end{array}$

$\begin{array}{lll}-2.76212900 & 0.08485500 & 2.60224300\end{array}$

$\begin{array}{lll}2.14171700 & -0.07954600 & 2.81540200\end{array}$

$\begin{array}{lll}0.53798700 & 0.02929600 & -2.18020500\end{array}$

$\begin{array}{llll}3.34447900 & 1.83684500 & -1.16683200\end{array}$

$\begin{array}{llll}3.99960400 & 1.05424100 & -1.55869100\end{array}$

$\begin{array}{lll}2.55444900 & 2.00881100 & -1.90376600\end{array}$

$\begin{array}{llll}3.94244500 & 2.75400700 & -1.07279300\end{array}$

$\begin{array}{lll}3.92715800 & 1.29874800 & 1.20201900\end{array}$

$\begin{array}{lll}4.51455300 & 2.22702000 & 1.21762000\end{array}$

$\begin{array}{lll}3.60034400 & 1.11748200 & 2.22929900\end{array}$

$\begin{array}{llll}4.60791900 & 0.49587400 & 0.90783300\end{array}$

$\begin{array}{llll}2.75571300 & 1.47739800 & 0.21642500\end{array}$

$\begin{array}{lll}1.90307800 & 2.67299900 & 0.68235400\end{array}$

$\begin{array}{lll}1.08636700 & 2.87445200 & -0.01559200\end{array}$

$\begin{array}{lll}1.47857500 & 2.51846700 & 1.67874100\end{array}$

$\begin{array}{lll}2.53447400 & 3.57084300 & 0.72100100\end{array}$

$\begin{array}{lll}2.57036200 & -1.68561900 & 0.06108700\end{array}$

$3.16766700-2.07728600 \quad 1.42624000$

$\begin{array}{lll}2.39031300 & -2.25769200 & 2.17383600\end{array}$

$\begin{array}{lll}3.72619700 & -3.01580200 & 1.30742700\end{array}$

$\begin{array}{lll}3.86546000 & -1.33618900 & 1.82393000\end{array}$

$\begin{array}{lll}3.69898500 & -1.62750400 & -0.99060700\end{array}$

$\begin{array}{lll}4.13624800 & -2.62898900 & -1.09948400\end{array}$

$3.31759600-1.31822900-1.96981200$

$\begin{array}{lll}4.50947700 & -0.95058300 & -0.70476000\end{array}$

$\begin{array}{lll}1.59918900 & -2.80727200 & -0.35859900\end{array}$

$\begin{array}{lll}0.77895600 & -2.92119800 & 0.35566600\end{array}$

$\begin{array}{lll}1.17002600 & -2.61744900 & -1.34757800\end{array}$ $2.14095900-3.76235000-0.39463400$
\end{abstract}

XPhos distortion for complexation: $6.46 \mathrm{kcal} / \mathrm{mol}$

$\mathrm{PhBr}$

Electronic energy (M06): -244.6591789

PhBr@tBuXPhos_preOA TS geom

Electronic energy (M06): -244.6489646

Distortion for complexation with PdtBuXPhos: $6.41 \mathrm{kcal} / \mathrm{mol}$

PhBr@XPhos_preOA_TS_geom

Electronic energy (M06): -244.6508591

Distortion for complexation with PdtBuXPhos:5.22kcal $/ \mathrm{mol}$ 